\title{
A dichotomy of sets via typical differentiability
}

\author{
Michael Dymond ${ }^{1}$ and Olga Maleva ${ }^{2}$ \\ ${ }^{1}$ Institut für Mathematik, Universität Innsbruck, Technikerstraße 13, 6020 Innsbruck, Austria; \\ E-mail: Michael.Dymond@uibk.ac.at. \\ ${ }^{2}$ School of Mathematics, University of Birmingham, Birmingham, B15 2TT United Kingdom; E-mail: O.Maleva@bham.ac.uk.
}

Received: 2 October 2019; Revised: 18 May 2020; Accepted: 26 May 2020

2020 Mathematics Subject Classification: Primary-26B05, 26A16; Secondary-28A05, 26A21, 03E15, 28A75

Keywords and phrases: differentiability of Lipschitz functions; Baire category; purely unrectifiable; Banach-Mazur game

\begin{abstract}
We obtain a criterion for an analytic subset of a Euclidean space to contain points of differentiability of a typical Lipschitz function: namely, that it cannot be covered by countably many sets, each of which is closed and purely unrectifiable (has a zero-length intersection with every $C^{1}$ curve). Surprisingly, we establish that any set failing this criterion witnesses the opposite extreme of typical behaviour: in any such coverable set, a typical Lipschitz function is everywhere severely non-differentiable.
\end{abstract}

\section{Contents}

1 Introduction $\quad 1$

2 Main results 4

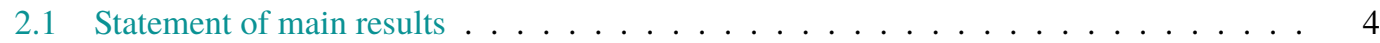

2.2 Strategy of the proof of typical differentiability $\ldots \ldots \ldots \ldots$

2.3 Application in universal differentiability set theory . . . . . . . . . . . 6

3 Typical differentiability inside Lipschitz curves $\quad 7$

4 Curve detection of non-coverable sets $\quad 22$

5 Typical non-differentiability on coverable sets $\quad 36$

6 Comparison with vector-valued mappings $\quad 38$

\section{Introduction}

Whilst the classical Rademacher's theorem guarantees that every set of positive (outer) Lebesgue measure in a Euclidean space $\mathbb{R}^{d}$ contains points of differentiability of every Lipschitz function on $\mathbb{R}^{d}$, a major direction in geometric measure theory research in the last two decades was to explore to what extent this is true for Lebesgue null subsets of $\mathbb{R}^{d}$. It was shown in the 1940s [3, 25] that for any null set $N \subseteq \mathbb{R}$, there is a Lipschitz function $f: \mathbb{R} \rightarrow \mathbb{R}$ nowhere differentiable in $N$. In contrast, for any $d \geq 2$, there are Lebesgue null sets in which every Lipschitz function $\mathbb{R}^{d} \rightarrow \mathbb{R}$ has points of differentiability; see [20, 5, 7]. Sets with the latter property are called universal differentiability sets (UDSs).

(C) The Author(s), 2020. Published by Cambridge University Press. This is an Open Access article, distributed under the terms of the Creative Commons Attribution licence (http://creativecommons.org/licenses/by/4.0/), which permits unrestricted re-use, distribution, and reproduction in any medium, provided the original work is properly cited. 
But if there is a Lipschitz function nowhere differentiable on a given set $N$, one naturally wonders what happens with a typical (in the sense of Baire category - see the exact definition below) Lipschitz function on $N$. Classical results suggest that typical functions exhibit the worst possible differentiability behaviour: e.g. a typical continuous function on an interval is nowhere differentiable; see [2]. Surprisingly, the complete opposite may be true in spaces of Lipschitz functions, even in spaces of Lipschitz functions restricted to some non-UDS $N$. In dimension one, [22] shows that $N \subseteq \mathbb{R}$ can be covered by a countable union of closed null sets if and only if a typical 1-Lipschitz function $\mathbb{R} \rightarrow \mathbb{R}$ has no points of differentiability in $N$. It can be seen from the proof in [22] that for all other analytic sets, a typical 1-Lipschitz function will be differentiable at a point inside the set.

In the present paper, we settle the question of differentiability of a typical Lipschitz function inside a given analytic subset $N$ of $\mathbb{R}^{d}, d \geq 2$. We give a complete characterisation of the subsets $N$ of $\mathbb{R}^{d}$ in which a typical 1-Lipschitz function has points of differentiability: they cannot be covered by an $F_{\sigma}$ purely unrectifiable set; we refer to such sets as typical differentiability sets (a simple example is a $C^{1}$-curve in $\mathbb{R}^{d}$ ). We also show that for all remaining sets $N$, a typical 1-Lipschitz function is nowhere differentiable, even directionally, inside $N$.

We formally state our main results in the next section; see Theorems 2.1 and 2.2, which imply a dichotomy between typical differentiability and typical non-differentiability sets for every dimension $d \geq 1$ (see Corollary 2.3).

Note that universal differentiability sets form a subclass of typical differentiability sets. Although to date there is no geometric-measure criterion for a set to be a UDS, it has been established that UDSs may be extremely small, e.g. compact and have Minkowski dimension 1; see [7]. This demonstrates the extent to which the $F_{\sigma}$-null criterion from [22] fails in higher dimensions: in dimension one, countable unions of closed null sets are typically non-differentiability sets; but in all higher dimensions, they may actually capture differentiability points of every Lipschitz function. We expect that, in the same spirit as for UDSs, typical differentiability sets will be explored further, in particular providing insight into typical behaviour of Lipschitz functions on non-Euclidean spaces; in this context, one should mention recent research into UDSs in Heisenberg and, more generally, Carnot groups $[21,19,14]$.

Let us be more precise about the terminology we use. The present paper will not be excessively concerned with the measurability of subsets of Euclidean spaces, so we will use the term measure in the sense of Hausdorff measure, as in [16]. This includes both the Lebesgue and outer Lebesgue onedimensional measure, which we denote by $\mathcal{L}$. A Lipschitz mapping with Lipschitz constant less than or equal to one is referred to as 1 -Lipschitz; let $\operatorname{Lip}_{1}\left([0,1]^{d}\right)$ denote the set of all 1-Lipschitz functions $f:[0,1]^{d} \rightarrow \mathbb{R}$, viewed as a complete metric space when equipped with the metric $\rho(f, g)=\|g-f\|_{\infty}$. For any Lipschitz mapping $f$, let $\operatorname{Diff}(f)$ denote the set of $t$ such that $f$ is differentiable at $t$. We say that a typical 1-Lipschitz function has a certain property if the set of those $f \in \operatorname{Lip}_{1}\left([0,1]^{d}\right)$ with this property is a residual subset of $\operatorname{Lip}_{1}\left([0,1]^{d}\right)$, i.e. its complement is meagre (in other words, is of first category).

We refer to a set $S \subseteq(0,1)^{d}$ as a typical differentiability set if a typical 1-Lipschitz function has points of differentiability in $S$, i.e. $\operatorname{Diff}(f) \cap S \neq \emptyset$. Let us also refer to subsets of $(0,1)^{d}$ in which a typical 1-Lipschitz function has no points of differentiability as typical non-differentiability sets. A priori, a set $S \subseteq(0,1)^{d}$ may have exactly one of these two properties, or none; we show in Corollary 2.3 that for analytic $S$, 'none' is impossible.

We would like to add that a very recent advance in this area, primarily for vector-valued Lipschitz mappings to Euclidean spaces of at least the same dimension, was made by Merlo [17].

It is worth mentioning further specific details of the aforementioned works [22] and [17] that are of relevance to the present paper. Recall that [22] characterises typical non-differentiability sets in $[0,1]$ as those sets that can be covered by countably many closed sets of measure zero. It also gives a sufficient condition for a set to be a typical differentiability set via the property of having 'every portion of positive measure'. We now give a definition of this notion and its higher-dimensional analogue. 


\section{Definition 1.1.}

(i) We say that a set $F \subseteq \mathbb{R}$ has every portion of positive measure if for every open set $U \subseteq \mathbb{R}$ with $U \cap F \neq \emptyset$, we have that $\mathcal{L}(U \cap F)$ is positive.

(ii) We say that a set $\mathscr{F} \subseteq \mathbb{R}^{d}$ has every portion of positive cone width if for every open set $U \subseteq \mathbb{R}^{d}$ with $U \cap \mathscr{F} \neq \emptyset$, there exists a $C^{1}$-smooth curve $v_{U}:[0,1] \rightarrow \mathbb{R}^{d}$ with nowhere zero derivative such that $\mathcal{L}\left(v_{U}^{-1}(U \cap \mathscr{F})\right)$ is positive.

Remark 1.2. If a set $\mathscr{F}$ has every portion of positive cone width and $a>0$, then the curve $v_{U}$ may always be chosen so that it additionally satisfies $\left\|v_{U}^{\prime}(t)\right\|=a$ for all $t \in(0,1)$.

Also, in Section 4, we introduce the notation $\Gamma_{\mathscr{F}}(U)$ to denote the collection of all $C^{1}$-smooth curves $\gamma$ with codomain $U$ and $\mathcal{L}\left(\gamma^{-1}(\mathscr{F})\right)>0$. We may note here that if $\mathscr{F}$ has every portion of positive cone width, the set $U$ is open with $U \cap \mathscr{F} \neq \emptyset$, and $a>0$, then there exists $v_{U} \in \Gamma_{\mathscr{F}}(U)$ such that $\left\|v_{U}^{\prime}(t)\right\|=a$ for all $t$.

Note that the two notions (i) and (ii) coincide in dimension $d=1$. Petruska [18, Theorem 1] proves that analytic subsets of $[0,1]$ not coverable by a union of countably many closed, measure zero sets can be characterised as those sets $E \subseteq[0,1]$ for which there exists a closed set $F \subseteq[0,1]$ having every portion of positive measure such that $E \cap F$ is relatively residual in $F$.

Definition 1.3. We will use the term Lipschitz curve to refer to a Lipschitz mapping $\gamma: I \rightarrow \mathbb{R}^{d}$, where $I \subseteq \mathbb{R}$ is a closed interval, with the property that the derivative $\gamma^{\prime}$ is bounded away in magnitude from zero almost everywhere.

A set $P \subseteq \mathbb{R}^{d}$ is said to be purely unrectifiable if for every Lipschitz curve $\gamma:[0,1] \rightarrow \mathbb{R}^{d}$, the set $\gamma^{-1}(P)$ has Lebesgue measure zero.

The class of purely unrectifiable sets is widely regarded as the most exceptional in relation to differentiability of Lipschitz functions. Moreover, recently Máthe has announced that, within the class of Borel sets, purely unrectifiable sets coincide with the formally smaller class of uniformly purely unrectifiable sets (see [15], Definition 1.4 and Remark 1.7). Alberti, Csörnyei, and Preiss prove in [1] that any uniformly purely unrectifiable set $P \subseteq \mathbb{R}^{d}$ admits a Lipschitz function $f: \mathbb{R}^{d} \rightarrow \mathbb{R}$ that fails to have any directional derivatives in the set $P$. A strengthening of this is proved by Maleva and Preiss in [15, Theorem 1.13]: such a function $f$ may be constructed so that at all $x \in P$, the function $f$ is non-differentiable at $x$ in the strongest possible sense:

$$
\liminf _{r \rightarrow 0} \sup _{\|y\| \leq r} \frac{|f(x+y)-f(x)-\langle e, y\rangle|}{r}=0
$$

for every $e \in \mathbb{R}^{d}$ with $\|e\| \leq 1$. This condition expresses that every linear mapping $\mathbb{R}^{d} \rightarrow \mathbb{R}$ of norm at most one behaves as the derivative of $f$ along a certain subsequence approaching $x$. In Section 5, we show that the results of [15] are extremely relevant to typical non-differentiability; see Theorem 2.7 .

To find a characterisation of typical differentiability sets in higher-dimensional Euclidean spaces, one might seek higher-dimensional analogues of interval subsets not coverable by unions of countably many closed null sets. However, as explained earlier, the same notion cannot work, in particular because there are closed, null universal differentiability sets. We verify that countable unions of closed purely unrectifiable sets, which coincide with countable unions of closed null sets in the case $d=1$, are the fitting choice; see the characterisation given in Theorems 2.1 and 2.2. Merlo [17] also proposes that the correct higher-dimensional analogues of typical non-differentiability sets for vector-valued Lipschitz mappings are those subsets of $[0,1]^{d}$ that can be covered by a union of countably many closed, purely unrectifiable sets. 


\section{Main results}

\subsection{Statement of main results}

In the present section, we set out the structure of the proof of our main results, Theorems 2.1 and 2.2:

Theorem 2.1. Let $d \geq 1$. The following are equivalent for an analytic set $\mathscr{A} \subseteq(0,1)^{d}$ :

(a) The set $\mathscr{A}$ cannot be covered by an $F_{\sigma}$, purely unrectifiable set.

(b) A typical $f \in \operatorname{Lip}_{1}\left([0,1]^{d}\right)$ has points of differentiability in $\mathscr{A}$, i.e. $\mathscr{A}$ is a typical differentiability subset of $(0,1)^{d}$.

Theorem 2.2. Let $d \geq 1$. The following are equivalent for an analytic set $\mathscr{A} \subseteq(0,1)^{d}$ :

(i) The set $\mathscr{A}$ is contained in an $F_{\sigma}$, purely unrectifiable set.

(ii) A typical $f \in \operatorname{Lip}_{1}\left([0,1]^{d}\right)$ is nowhere differentiable in $\mathscr{A}$, i.e. $\mathscr{A}$ is a typical non-differentiability subset of $(0,1)^{d}$.

We caution again that Theorems 2.1 and 2.2 are not formally equivalent statements, i.e. the negation of (ii) is not formally the same as (b). Thus, the following dichotomy is also a new result that follows from Theorems 2.1 and 2.2.

Corollary 2.3. Let $d \geq 1$. Each analytic subset $\mathscr{A} \subseteq(0,1)^{d}$ belongs to exactly one of the following two classes: typical differentiability or typical non-differentiability sets.

Remark 2.4. Note that a typical differentiability set $\mathscr{A}$ may be purely unrectifiable. As an example, we may take $\mathscr{A}$ as a 1 -dimensional, Lebesgue null, $G_{\delta}$ set dense in $[0,1]$, embedded in $[0,1]^{d}$. Although by [15, Theorem 1.13], there is a Lipschitz function non-differentiable in $\mathscr{A}$ in the strongest possible sense, Theorem 2.1 guarantees that a typical Lipschitz function has differentiability points in $\mathscr{A}$.

Theorems 2.1 and 2.2 in dimension $d=1$ coincide with the results proved by Preiss and Tišer in [22]; in this paper, we provide a proof of the two statements for all dimensions $d \geq 1$. Also, as a corollary of the proof of Theorem 2.1, we obtain a strengthening of their typical differentiability result; see Remark 2.9.

Since conditions (a) of Theorem 2.1 and (i) Theorem 2.2 are mutually exclusive, it is enough to prove only the implications (a) $\Rightarrow$ (b) in Theorem 2.1 and (i) $\Rightarrow$ (ii) in Theorem 2.2. For convenience, we restate these as two new statements. Moreover, we include in these two statements additional details concerning special forms of differentiability and non-differentiability that, for simplicity, are omitted from Theorems 2.1 and 2.2.

Theorem 2.5. Let $d \geq 1$. If an analytic set $\mathscr{A} \subseteq(0,1)^{d}$ cannot be covered by an $F_{\sigma}$, purely unrectifiable set, then a typical $f \in \operatorname{Lip}_{1}\left([0,1]^{d}\right)$ has points of differentiability in $\mathscr{A}$. Such points $x \in \mathscr{A}$ may additionally be taken so that the gradient $\nabla f(x)$ of $f$ at $x$ has magnitude one.

In Theorem 2.7, we show that the non-differentiability of Theorem 2.2 may be taken in a stronger sense. Namely, we prove that for each typical non-differentiability set $\mathscr{A}$, a typical function $f \in$ $\operatorname{Lip}_{1}\left([0,1]^{d}\right)$ has no directional derivatives at every $x \in \mathscr{A}$; moreover, its derived set $\mathcal{D} f(x, v)$, defined below, coincides with $[-1,1]$ for each $\|v\|=1$.

Definition 2.6. Suppose that $f:[0,1]^{d} \rightarrow \mathbb{R}$ is a function and $x \in(0,1)^{d}, v \in \mathbb{S}^{d-1}$ are two vectors. The derived set of $f$ at the point $x$ in the direction of $v$ is defined as the set $\mathcal{D} f(x, v)$ of all existing limits $\lim _{n \rightarrow \infty}\left(f\left(x+t_{n} v\right)-f(x)\right) / t_{n}$, where $t_{n} \searrow 0$.

Theorem 2.7. Let $d \geq 1$. If a set $\mathscr{A} \subseteq(0,1)^{d}$ can be covered by an $F_{\sigma}$, purely unrectifiable set, then a typical $f \in \operatorname{Lip}_{1}\left([0,1]^{d}\right)$ has no directional derivatives at every point of $\mathscr{A} ;$ moreover, for a typical $f \in \operatorname{Lip}_{1}\left([0,1]^{d}\right)$, it holds that $\mathcal{D} f(x, v)=[-1,1]$ for every $x \in \mathscr{A}$ and every $v \in \mathbb{S}^{d-1}$.

To conclude, note that [17] provides a statement analogous to Theorem 2.5 in spaces of vector-valued Lipschitz mappings $\mathbb{R}^{d} \rightarrow \mathbb{R}^{m}$, with the restriction $m \geq d$, and with only directional differentiability instead of full differentiability. Although this statement might appear similar in spirit, we show in 
Section 6 that projection arguments do not allow one to lower the codomain dimension to 1 , as we achieve in Theorem 2.5. On the other hand, parts of the argument employed in [17] apply to Lipschitz mappings without restriction on the dimension of the codomain, and therefore Theorem 2.7 is proved implicitly there. However, in Section 5 of the present article, we provide an independent shorter proof of Theorem 2.7, using results of [15].

\subsection{Strategy of the proof of typical differentiability}

The proof of the 'typical differentiability' Theorem 2.5 roughly divides into two halves, proved in Sections 3 and 4. In the first part, we prove the statement for the special case where $\mathscr{A}$ (or $\gamma(E)$ in the statement below) is a subset of a Lipschitz curve with unique tangents at all points in $\mathscr{A}$.

Theorem 2.8. Let $\emptyset \neq F \subseteq[0,1]$ be a closed set with every portion of positive measure, and let $E$ be a relatively residual subset of $F$. Let $\gamma:[0,1] \rightarrow(0,1)^{d}$ be a Lipschitz curve with Lipschitz constant 1 , such that $\gamma$ is differentiable with derivative of magnitude one at each $t \in E$. Then the set $S$ of those functions $f \in \operatorname{Lip}_{1}\left([0,1]^{d}\right)$ for which there exists $t \in E$ such that $f$ is differentiable with derivative of magnitude one at $\gamma(t)$ is residual in $\operatorname{Lip}_{1}\left([0,1]^{d}\right)$.

Remark 2.9. In the setting of Theorem 2.8, it is possible to obtain the stronger conclusion that there is a residual subset $R$ of $\operatorname{Lip}_{1}\left([0,1]^{d}\right)$ for which every function $f \in R$ is differentiable at $\gamma(t)$ for residually many $t \in F$ (or, equivalently, for residually many $t \in E$ ). Loosely rephrased, a typical $f \in \operatorname{Lip}_{1}\left([0,1]^{d}\right)$ is differentiable at a typical point of $\gamma(F)$ (or a typical point of $\gamma(E)$ ). For further details, see Remark 3.18.

Importantly, this is a new observation even in dimension $d=1$, where it asserts a stronger property of one-dimensional typical differentiability sets than that proved in [22]; in particular, it strengthens [22, Lemma 2]. Indeed, we may state the following extension of the results of [22]:

If an analytic set $\mathscr{A} \subseteq[0,1]$ cannot be covered by a one-dimensional Lebesgue null $F_{\sigma}$ set, then there exists a non-empty closed set $F \subseteq[0,1]$ with every portion of positive measure and a residual set of functions $f \in \operatorname{Lip}_{1}\left([0,1]^{d}\right)$ for which $\mathscr{A} \cap \operatorname{Diff}(f)$ is a residual subset of $F$. The same conclusion holds for any non-empty closed set $F \subseteq[0,1]$ with every portion of positive measure such that $\mathscr{A} \cap F$ is residual in $F$.

Theorem 2.8 is proved in Section 3. Then, in Section 4, we show that the general statement of Theorem 2.5 reduces to the special case of Theorem 2.8. Put differently, we show that any set $\mathscr{A} \subseteq(0,1)^{d}$ satisfying the hypotheses of Theorem 2.5 intersects some $\operatorname{Lipschitz}$ curve $\gamma$, with $\operatorname{Lip}(\gamma) \leq 1$, in the particular manner required by Theorem 2.8. To achieve this, we make important use of the following proposition, which follows from [24]; compare with [17, Theorem 2.8]. It shows that analytic sets that cannot be covered by a countable union of closed purely unrectifiable sets may be approximated by closed sets having every portion of positive cone width; see Definition 1.1 (ii).

Proposition 2.10. If an analytic set $\mathscr{A} \subseteq(0,1)^{d}$ cannot be covered by a countable union of closed purely unrectifiable sets, then there exists a closed set $\mathscr{F} \subseteq[0,1]^{d}$ such that $\mathscr{A} \cap \mathscr{F}$ is residual in $\mathscr{F}$ and $\mathscr{F}$ has every portion of positive cone width.

Proof. We apply [24, Remark (2), page 1024] to the collection $I$ of all closed, purely unrectifiable sets and set $\mathscr{A}$. We see that if $\mathscr{A}$ cannot be covered by a countable union of closed, purely unrectifiable sets, i.e. $\mathscr{A} \notin I_{\text {ext }}$, then there is a non-empty closed set $\mathscr{F}=C$ such that $\mathscr{A} \cap \mathscr{F}$ contains a $G_{\delta}$ set dense in $\mathscr{F}$ (implying that $\mathscr{A} \cap \mathscr{F}$ is residual in $\mathscr{F}$ ), and such that for any open set $V$ with $V \cap \mathscr{F} \neq \emptyset$, it holds that $\overline{V \cap \mathscr{F}} \notin I$. In other words, $\overline{V \cap \mathscr{F}}$ is not a purely unrectifiable set, which implies that there exists a $C^{1}$-smooth curve $\gamma$ such that $\mathcal{L}\left(\gamma^{-1}(\overline{V \cap \mathscr{F}})\right)>0$, implying $\mathcal{L}\left(\gamma^{-1}(\bar{V} \cap \mathscr{F})\right)>0$. Let $U$ be an open set with $U \cap \mathscr{F} \neq \emptyset$, and let $x \in U \cap \mathscr{F}$. Choose $r>0$ such that $V=B(x, r) \subseteq \bar{V} \subseteq U$. If we take $v=\gamma$ as above, the condition of Definition 1.1 (ii) is satisfied for $U$, and the statement follows.

With Proposition 2.10 at hand, the reduction to the 'special case' described above is completed by the next theorem. 
Theorem 2.11. Let $d \geq 1$ and $\mathscr{F} \subseteq[0,1]^{d}$ be a non-empty, closed set having every portion of positive cone width. Let $\mathscr{A} \subseteq(0,1)^{d}$ be an analytic set such that $\mathscr{A} \cap \mathscr{F}$ is relatively residual in $\mathscr{F}$. Then there exists a 1-Lipschitz curve $\gamma:[0,1] \rightarrow(0,1)^{d}$ and sets $E \subseteq F \subseteq[0,1]$ with the following properties:

(i) $F$ is non-empty and closed and has every portion of positive measure.

(ii) $E$ is residual in $F$.

(iii) $\gamma$ is differentiable at every point $t \in E$ with $\left\|\gamma^{\prime}(t)\right\|=1$.

(iv) For every $t \in E$, we have

$$
\lim _{\delta \rightarrow 0} \operatorname{osc}_{\gamma^{\prime}}([t-\delta, t+\delta])=0 .
$$

(v) $\gamma(E) \subseteq \mathscr{A}$.

The quantity osc $\gamma_{\gamma^{\prime}}([t-\delta, t+\delta])$ of (iv) should be understood in the natural way; for a more precise definition, see Section 4, (4.1).

Remark 2.12. We point out that Theorem 2.11 and Proposition 2.10 combine to give the following statement, which may be viewed as a generalisation of the one-dimensional result of [18] to all higher dimensions:

An analytic set $\mathscr{A} \subseteq(0,1)^{d}$ cannot be covered by a countable union of closed, purely unrectifiable sets if and only if there exists a 1 -Lipschitz curve $\gamma:[0,1] \rightarrow(0,1)^{d}$ and a non-empty, closed set $F \subseteq[0,1]$ having every portion of positive measure such that $\gamma^{-1}(\mathscr{A}) \cap \operatorname{Diff}(\gamma)$ intersects $F$ in a relatively residual set.

To prove Theorem 2.11, we construct a sequence $\left(\gamma_{k}\right)_{k=1}^{\infty}$ of Lipschitz curves $\gamma_{k}$ converging uniformly to the desired curve $\gamma$. We postpone this construction until Section 4. For now, let us present a proof of Theorem 2.5 based on Theorems 2.8 and 2.11 and Proposition 2.10.

Proof of Theorem 2.5. By Proposition 2.10, there exists a closed set $\mathscr{F} \subseteq[0,1]^{d}$ such that $\mathscr{A}$ and $\mathscr{F}$ satisfy the conditions of Theorem 2.11. Let $\gamma, E$, and $F$ be given by the conclusion of Theorem 2.11. Then $\gamma, E$, and $F$ satisfy the conditions of Theorem 2.8. Applying Theorem 2.8, we conclude that a typical $f \in \operatorname{Lip}_{1}\left([0,1]^{d}\right)$ has points of differentiability where the derivative has magnitude one in $\gamma(E) \subseteq \mathscr{A}$.

\subsection{Application in universal differentiability set theory}

Recall $[1,15]$ that purely unrectifiable sets fail badly to have the universal differentiability property. However, there are examples that show that such sets may provide surprisingly many differentiability points of some Lipschitz functions. Csörnyei, Preiss, and Tišer construct in [4] a universal differentiability set $E \subseteq \mathbb{R}^{2}$, a purely unrectifiable subset $P \subseteq E$, and a Lipschitz function $h: \mathbb{R}^{2} \rightarrow \mathbb{R}$ such that all differentiability points of $h$ in the universal differentiability set $E$ are captured by $P$, that is,

$$
\operatorname{Diff}(h) \cap E \subseteq P .
$$

In the new paper [6], Dymond shows that by a modification of this construction, the set $P$ may additionally capture all differentiability points in $E$ of a typical Lipschitz function in the shifted Lip 1 space $X=h+\operatorname{Lip}_{1}\left([0,1]^{2}\right)$. In other words, (2.1) holds not just for $h$, but also for a typical $f \in X$. This naturally invites the question of whether it is possible to find $E$ and $P$ so that (2.1) holds for a typical $f$ in the natural space $\operatorname{Lip}_{1}\left([0,1]^{d}\right)$ without any shift. As an application of the dichotomy between typical differentiability and typical non-differentiability sets (see Theorems 2.1 and 2.2 and Corollary 2.3), we establish that this is not possible. Although Theorem 2.13 shows that purely unrectifiable sets cannot capture all points of differentiability of a typical Lipschitz function within a given universal differentiability set, the main result of [6] asserts that purely unrectifiable sets may nonetheless capture 'equivalently' large sets of differentiability points of a typical Lipschitz function. 
Theorem 2.13. Let $U \subseteq[0,1]^{d}$ be a universal differentiability set and $V \subseteq U$ be a subset with the property that

$$
\operatorname{Diff}(f) \cap U \subseteq V
$$

for a typical $f \in \operatorname{Lip}_{1}\left([0,1]^{d}\right)$. Then $V$ is not a purely unrectifiable set.

Proof. By assumption, the set $U \backslash V$ is a typical non-differentiability set. Hence, Theorem 2.2 implies that the set $U \backslash V$ is purely unrectifiable. If we assume that $V$ is also purely unrectifiable, we conclude that their union $U$ is purely unrectifiable and hence a cone unrectifiable set; see [15, Definition 1.7 and Remark 1.8]. Applying [15, Theorem 1.1] to the set $U$, we obtain a Lipschitz function $g$ that is non-differentiable everywhere in $U$, contrary to $U$ being a universal differentiability set.

\section{Typical differentiability inside Lipschitz curves}

In this section, we prove Theorem 2.8 .

Definition 3.1. Let $\gamma:[0,1] \rightarrow(0,1)^{d}$ be a Lipschitz curve and $F \subseteq[0,1]$ be a closed set. We say that $\gamma$ is affine modulo $F$ if $\gamma$ is affine on each component of $[0,1] \backslash F$.

The next lemma allows us to assume that the Lipschitz curve given by the hypothesis of Theorem 2.8 is affine modulo $F$.

Lemma 3.2. If $\gamma:[0,1] \rightarrow(0,1)^{d}$ is a Lipschitz curve, $F \subseteq[0,1]$ is a closed set, $E \subseteq F$ is a relatively residual subset of $F$, and $\gamma^{\prime}(t)$ exists for every $t \in E$, then we may redefine $\gamma$ and $E$ as $\gamma_{1}$ and $E_{1}$ in such a way that $E_{1} \subseteq E$ is a relatively residual subset of $F, \gamma_{1}:[0,1] \rightarrow(0,1)^{d}$ is a Lipschitz curve with $\operatorname{Lip}\left(\gamma_{1}\right) \leq \operatorname{Lip}(\gamma), \gamma_{1}(t)=\gamma(t)$ for $t \in E_{1}, \gamma_{1}$ is differentiable at every $t \in E_{1}$ with $\gamma_{1}^{\prime}(t)=\gamma^{\prime}(t)$, and $\gamma_{1}$ is affine modulo $F$.

Proof. Note that $(0,1) \backslash F$ is an open set; hence it is equal to the union $\bigcup_{n=1}^{\infty}\left(a_{n}, b_{n}\right)$ of open, disjoint intervals. Let $E_{1}=E \backslash \bigcup_{n \geq 1}\left\{a_{n}, b_{n}\right\}$; redefine $\gamma$ on each of $\left(a_{n}, b_{n}\right)$ in an affine way, and call the new curve $\gamma_{1}$. Note that $E_{1}$ is a relatively residual subset of $F$ and that $\gamma_{1}:[0,1] \rightarrow(0,1)^{d}$ is a Lipschitz curve with $\operatorname{Lip}\left(\gamma_{1}\right) \leq \operatorname{Lip}(\gamma)$ and $\gamma_{1}(t)=\gamma(t)$ for all $t \in E_{1}$.

To check that $\gamma_{1}$ is differentiable on $E_{1}$, let us fix any $t \in E_{1}$ and $\varepsilon>0$. As $\gamma$ is differentiable at $t$, let $v \in \mathbb{R}^{d}$ and $\delta>0$ be such that $\|\gamma(t+h)-\gamma(t)-h v\| \leq \varepsilon|h|$ for all $|h|<\delta$. Let $N=$ $\left\{n \geq 1:\left(b_{n}-a_{n}\right) \geq \delta / 2\right\}$. Note that the set $N$ is finite, and $t$ has positive distance from the set $U=\bigcup_{n \in N}\left(a_{n}, b_{n}\right)$. Let $\delta_{1}=\min (\operatorname{dist}(t, U), \delta / 2)$, and assume $|h|<\delta_{1}$. If $t+h \notin \bigcup_{n=1}^{\infty}\left(a_{n}, b_{n}\right)$, then $\gamma_{1}(t+h)=\gamma(t+h)$ and $\gamma_{1}(t)=\gamma(t)$, so that

$$
\left\|\gamma_{1}(t+h)-\gamma_{1}(t)-h v\right\| \leq \varepsilon|h| .
$$

If $n \geq 1$ is such that $t+h \in\left(a_{n}, b_{n}\right)$, then $n \notin N$, i.e. $\left(b_{n}-a_{n}\right)<\delta / 2$. Hence, using $|h|<\delta / 2$, we get $\left|a_{n}-t\right|,\left|b_{n}-t\right|<\delta$. We thus have, using $\gamma_{1}\left(a_{n}\right)=\gamma\left(a_{n}\right)$ and $\gamma_{1}\left(b_{n}\right)=\gamma\left(b_{n}\right)$, that

$$
\left\|\gamma_{1}\left(a_{n}\right)-\gamma_{1}(t)-\left(a_{n}-t\right) v\right\| \leq \varepsilon\left|a_{n}-t\right| \text { and }\left\|\gamma_{1}\left(b_{n}\right)-\gamma_{1}(t)-\left(b_{n}-t\right) v\right\| \leq \varepsilon\left|b_{n}-t\right| .
$$

As $t \notin\left[a_{n}, b_{n}\right]$, we have that either both $\left(a_{n}-t\right)$ and $\left(b_{n}-t\right)$ are positive or both are negative. Thus if $t+h=\alpha a_{n}+(1-\alpha) b_{n}$, for $\alpha \in(0,1)$, then

$$
\begin{aligned}
& \left\|\gamma_{1}(t+h)-\gamma_{1}(t)-h v\right\|=\left\|\alpha \gamma_{1}\left(a_{n}\right)+(1-\alpha) \gamma_{1}\left(b_{n}\right)-\gamma_{1}(t)-h v\right\| \\
& \leq \alpha\left\|\gamma_{1}\left(a_{n}\right)-\gamma_{1}(t)-\left(a_{n}-t\right) v\right\|+(1-\alpha)\left\|\gamma_{1}\left(b_{n}\right)-\gamma_{1}(t)-\left(b_{n}-t\right) v\right\| \\
& \leq \varepsilon\left|\alpha\left(a_{n}-t\right)+(1-\alpha)\left(b_{n}-t\right)\right|=\varepsilon|h|,
\end{aligned}
$$

verifying (3.1). 
Definition 3.3. Let $\gamma:[0,1] \rightarrow(0,1)^{d}$ be a Lipschitz curve, $I \subseteq[0,1]$ be an interval, $u \in \mathbb{S}^{d-1}$, and $\theta>0$. We say that $\gamma$ is $\theta$-flat in direction $u$ around $I$ if for all $t_{1}, t_{2} \in[0,1]$ with $\operatorname{dist}\left(t_{i}, I\right)<\mathcal{L}(I)$, it holds that

$$
\left\|\gamma\left(t_{1}\right)-\gamma\left(t_{2}\right)-\left(t_{1}-t_{2}\right) u\right\| \leq \theta\left|t_{1}-t_{2}\right|
$$

There are many cases when we do not need to keep information about the vector $u$. Thus we will often write simply that $\gamma$ is $\theta$-flat around $I$ to signify that $\gamma$ is $\theta$-flat around $I$ in some direction $u \in \mathbb{S}^{d-1}$.

Remark 3.4. Condition (3.2) is equivalent to the following: there exists $w_{t_{1}, t_{2}} \in \mathbb{R}^{d}$ with $\left\|w_{t_{1}, t_{2}}\right\| \leq 1$ such that

$$
\gamma\left(t_{1}\right)-\gamma\left(t_{2}\right)=\left(t_{1}-t_{2}\right)\left(u+\theta w_{t_{1}, t_{2}}\right) .
$$

Remark 3.5. It is not important whether the interval $I$ in the definition of $\theta$-flatness is open or closed: for $I_{1}=(a, b)$ and $I_{2}=[a, b]$, the values of $\mathcal{L}\left(I_{j}\right)$ and the sets of $t \in[0,1]$ such that $\operatorname{dist}\left(t, I_{j}\right)<\mathcal{L}\left(I_{j}\right)$ are the same.

Trivially, the flatness property passes to sub-intervals.

Notation. Given $t \in \mathbb{R}$ and $\delta>0$, we let

$$
I_{\delta}(t):=(t-\delta, t+\delta)
$$

Definition 3.6. Let $t \in \mathbb{R}, F \subseteq \mathbb{R}$, and $\varepsilon>0$. We say that $I_{\delta}(t)$ is an $\varepsilon$-density interval for $F$ if

$$
\mathcal{L}\left(I_{r}(t) \backslash F\right)<2 r \varepsilon \quad \text { for every } r \in(0, \delta] .
$$

Remark 3.7. Suppose $Y \subseteq \mathbb{R}$ is open and $X \subseteq Y$ has positive measure, and let $\varepsilon>0$. Then for almost all $t \in X$, there is an $\varepsilon$-density interval $I_{\delta}(t)$ for $X$ such that $I_{\delta}(t) \subseteq Y$. This follows from the Lebesgue Density theorem; see [16, Corollary 2.14 (1)].

Lemma 3.8. Let $\gamma:[0,1] \rightarrow(0,1)^{d}$ and $E \subseteq F \subseteq[0,1]$ satisfy the hypotheses of Theorem 2.8, and suppose that $\gamma$ is affine modulo $F$. Then for every interval $(a, b) \subseteq[0,1]$ for which $(a, b) \cap F \neq \emptyset$ and $\theta \in(0,1)$, there exist $u \in \mathbb{S}^{d-1}$ and an open interval $I \subseteq(a, b)$ such that $\gamma$ is $\theta$-flat in direction $u$ around $I$ and $I \cap F \neq \emptyset$.

Proof. Let $(a, b) \subseteq[0,1]$ with $(a, b) \cap F \neq \emptyset$. Choose a set $\left\{u_{k}\right\}$ of unit vectors, dense in the unit sphere $\mathbb{S}^{d-1}$, and consider a family of sets

$$
E_{k, m}=\left\{r \in[a, b]:\left\|\frac{\gamma(s)-\gamma(r)}{s-r}-u_{k}\right\| \leq \theta \quad \text { for all } s \in[a, b] \text { with } 0<|s-r|<1 / m\right\} .
$$

Note that each $E_{k, m}$ is closed and $\bigcup_{k, m} E_{k, m} \supseteq E \cap[a, b]$.

Since $E \cap[a, b]$ is relatively residual in $F \cap[a, b]$, there is a pair $(k, m)$ and a non-degenerate open interval $J \subseteq(a, b)$ such that $E_{k, m} \supseteq J \cap F \neq \emptyset$. Let $u=u_{k}, t \in J \cap F$, and choose $\Delta>0$ small enough that $I_{\Delta}(t) \subset J$. Let $0<\delta<\min (1 /(6 m), \Delta / 3)$. We show $I:=I_{\delta}(t)$ fulfils the assertions of the lemma. Since $t \in I \cap F$, we have $I \cap F \neq \emptyset$. We now verify the flatness of $\gamma$ around $I$ in direction $u$. Let $t_{1}, t_{2} \in[0,1]$ be such that $\operatorname{dist}\left(t_{i}, I\right)<\mathcal{L}(I)=2 \delta$. Then $\left|t_{1}-t_{2}\right|<6 \delta<1 / m$ and $t_{1}, t_{2} \in I_{\Delta}(t)$. If $t_{1} \in F$, then $t_{1} \in I_{\Delta}(t) \cap F \subseteq J \cap F \subseteq E_{k, m}$. Hence (3.2) is satisfied. Assume now $t_{1} \notin F$, and consider the decomposition of $I_{3 \delta}(t) \backslash F$ into the union of countably many disjoint open intervals $V_{n}=\left(a_{n}, b_{n}\right)$. We therefore have that $t_{1} \in V_{n}$ for some $n \geq 1$. If $t_{2} \in V_{n}$ too, then the affineness of $\gamma$ on $V_{n}$ and the fact that the endpoints of $V_{n}$ belong to $I_{3 \delta}(t) \cap F \subseteq J \cap F \subseteq E_{k, m}$ imply that (3.2) is satisfied. If $t_{2} \notin V_{n}$, then as $t_{2} \in I_{3 \delta}(t)$ and $V_{n} \subseteq I_{3 \delta}(t)$, we conclude that both $\left|a_{n}-t_{i}\right|$ and $\left|b_{n}-t_{i}\right|$ for $i=1,2$ are less than $6 \delta \leq 1 / m$. Hence, using $a_{n}, b_{n} \in I_{3 \delta}(t) \cap F \subseteq J \cap F \subseteq E_{k, m}$, we may write inequality (3.4) with $t_{2}$ and endpoints of $V_{n}$ to get (3.2) for $t_{1}, t_{2}$. 
Notation. Suppose $\gamma:[0,1] \rightarrow(0,1)^{d}$ is a 1-Lipschitz curve, $I \subseteq[0,1]$ is an interval, $P \subseteq I$ is finite, and $f:[0,1]^{d} \rightarrow \mathbb{R}$ is Lipschitz. Let $\sigma, \tau>0$, and consider the set $Y_{\sigma, I, P}=\{y \in$ $\left.[0,1]^{d}: \operatorname{dist}(y, \gamma(I)) \geq \sigma\right\} \cup \gamma(P)$. Denote

$$
\Phi_{\gamma, f, I, P, \sigma, \tau}(x)=\inf _{y \in Y_{\sigma, I, P}}(f(y)+\tau\|x-y\|), \quad x \in[0,1]^{d},
$$

and call $\Phi_{\gamma, f, I, P, \sigma, \tau}:[0,1]^{d} \rightarrow \mathbb{R}$ a conical function. If $\alpha \in(0,1)$ is a parameter and $\tau>1-\alpha$, we call $\Phi_{\gamma, f, I, P, \sigma, \tau}$ an $\alpha$-conical function.

Lemma 3.9. Let $f:[0,1]^{d} \rightarrow \mathbb{R}$ be a Lipschitz function, $\emptyset \neq Y \subseteq[0,1]^{d}$, and $\tau \geq \operatorname{Lip}(f)$. Then the conical function $\Phi(x)=\inf _{y \in Y}(f(y)+\tau\|x-y\|)$ is $\tau$-Lipschitz, and $\Phi(x)=f(x)$ for $x \in Y$.

Proof. For any $y \in Y$ and any $x \in[0,1]^{d}$, we have $f(y)-f(x) \geq-\operatorname{Lip}(f)\|x-y\|$, implying $f(y)+\tau\|x-y\| \geq f(x)+(\tau-\operatorname{Lip}(f))\|x-y\| \geq f(x)$, which means, for all $x \in[0,1]^{d}$,

$$
\Phi(x) \geq f(x) .
$$

In particular, the values of $\Phi$ are finite. As for each $y \in Y$, the function

$$
\varphi_{y}(x)=f(y)+\tau\|x-y\|
$$

is $\tau$-Lipschitz, and we conclude that $\Phi$ is $\tau$-Lipschitz, too. Note that additionally, for $x \in Y$, it trivially holds that $\Phi(x) \leq \varphi_{x}(x)=f(x)$. Thus $\Phi=f$ on $Y$.

Lemma 3.10. Let $\gamma:[0,1] \rightarrow(0,1)^{d}$ be a 1-Lipschitz curve that is $\theta$-flat around an interval $I \subseteq[0,1]$ in direction $u \in \mathbb{S}^{d-1}$, where $\theta \in(0,1 / 3)$. Let $\varepsilon>0$ and $f:[0,1]^{d} \rightarrow \mathbb{R}$ be a Lipschitz function with $\operatorname{Lip}(f)<1$. Then for every $\alpha \in(0,1)$ there is an $\alpha$-conical function, which we denote by $f_{\varepsilon, I}$, and $a$ closed, null set $N=N_{f, \varepsilon, I} \subseteq$ I with the following properties:

(i) $\operatorname{Lip}\left(f_{\varepsilon, I}\right)<1$ and $\left\|f_{\varepsilon, I}-f\right\|_{\infty}<\varepsilon$.

(ii) There is $\tau \in(1-\alpha, 1)$ such that for every component $J$ of $I \backslash N$ there is $p=p_{J} \in N$ such that

$$
f_{\varepsilon, I}(x)=f(\gamma(p))+\tau\|x-\gamma(p)\| \text { for all } x \in \gamma(J)
$$

and the function $f_{\varepsilon, I}$ is continuously differentiable on an open neighbourhood $U_{f}(\gamma(J))$ of $\gamma(J)$ with

$$
\nabla f_{\varepsilon, I}(x)=\tau \frac{x-\gamma(p)}{\|x-\gamma(p)\|} \quad \text { for all } x \in U_{f}(\gamma(J))
$$

Remark. Note that the conical function $f_{\varepsilon, I}$ and associated set $N_{f, \varepsilon, I}$ given by the conclusion of Lemma 3.10 depend on the value of $\tau$ and the curve $\gamma$. Since we will only ever consider conical functions with respect to a single fixed curve $\gamma$, we suppress this dependency on $\gamma$ in the notation. The value of $\tau$ will eventually be important for us, but we suppress it for now to keep the notation tidier.

Proof of Lemma 3.10. Set $\eta=\frac{\varepsilon(1-\operatorname{Lip}(f))}{64}, \sigma=\frac{\varepsilon}{8}$, fix any finite $\eta$-net $P$ of $I$, and let

$$
\tau \in\left(\max \left\{\frac{\operatorname{Lip}(f)+1}{2}, 1-\alpha\right\}, 1\right)
$$

be arbitrary. We define $f_{\mathcal{\varepsilon}, I}$ as the conical function $\Phi_{\gamma, f, I, P, \sigma, \tau}$ of (3.5). We will show that part (i) holds without further restriction on $\tau$, and that part (ii) holds with a suitable additional condition on $\tau$. 
By Lemma 3.9, the function $f_{\varepsilon, I}$ has Lipschitz constant less than or equal to $\tau<1$. If $x \in \mathbb{R}^{d}$ is such that $\operatorname{dist}(x, \gamma(I))<\sigma$, find $y \in \gamma(P)$ with $\|x-y\|<\sigma+\operatorname{Lip}(\gamma) \eta \leq \sigma+\eta$; then by Lemma 3.9, it follows that $f_{\varepsilon, I}(y)=f(y)$, so that

$$
\left|f_{\varepsilon, I}(x)-f(x)\right| \leq\left|f_{\varepsilon, I}(x)-f_{\varepsilon, I}(y)\right|+|f(y)-f(x)| \leq(\tau+1)(\sigma+\eta)<2 \frac{\varepsilon}{4}=\frac{\varepsilon}{2} .
$$

Hence, again using Lemma 3.9, we get $\left\|f_{\varepsilon, I}-f\right\|_{\infty}<\varepsilon$, completing (i) for all $\tau$ satisfying (3.8).

We now determine an additional mild restriction on $\tau$ satisfying (3.8), under which part (ii) is valid. Note first that (3.8) implies $\eta<\frac{\varepsilon(\tau-\operatorname{Lip}(f))}{32}$, from which it follows that $\sigma=\frac{\varepsilon}{8}>\frac{4 \tau \eta}{\tau-\operatorname{Lip}(f)}$. Consider any $x, y \in \mathbb{R}^{d}$ such that $\operatorname{dist}(x, \gamma(I))<\eta$ and $\operatorname{dist}(y, \gamma(I)) \geq \sigma$. Find $z \in \gamma(P)$ such that $\|x-z\| \leq \eta+\operatorname{Lip}(\gamma) \eta \leq 2 \eta$. Then, using additionally $\operatorname{Lip}(f)-\tau<0$ and $\|y-z\| \geq \sigma$, we get

$$
\begin{gathered}
f(z)-f(y)+\tau\|x-z\|-\tau\|x-y\| \leq \operatorname{Lip}(f)\|y-z\|+2 \tau \eta-\tau(\|y-z\|-2 \eta) \\
=(\operatorname{Lip}(f)-\tau)\|y-z\|+4 \tau \eta \leq(\operatorname{Lip}(f)-\tau) \sigma+4 \tau \eta<0
\end{gathered}
$$

so that $f(y)+\tau\|x-y\| \geq f(z)+\tau\|x-z\|$. Using the definition (3.5) of the conical function $f_{\varepsilon, I}=$ $\Phi_{\gamma, f, I, P, \sigma, \tau}$, we conclude that

$$
f_{\varepsilon, I}(x)=\min _{y \in \gamma(P)}(f(y)+\tau\|x-y\|), \quad \text { for all } x \in B(\operatorname{Im}(\gamma), \eta)
$$

Let $\Gamma=\{(y, z): y, z \in \gamma(P)$ and $y \neq z\}$ (a finite set). Fix a pair $(y, z) \in \Gamma$; then $y=\gamma(p) \neq z=\gamma(q)$, implying $p \neq q$, and let

$$
M_{y, z, \tau}=\{t \in I: f(y)+\tau\|\gamma(t)-y\|=f(z)+\tau\|\gamma(t)-z\|\} .
$$

Each $M_{y, z, \tau}$ is a closed subset of $I$. Note that the set $S_{y, z}$ of solutions $t \in I$ of $\|\gamma(t)-y\|=\|\gamma(t)-z\|$ cannot contain more than one point. Indeed, if $t_{1}, t_{2} \in S_{y, z}$ are distinct, then, as both $\gamma\left(t_{i}\right)$ are equidistant from $y$ and $z$, we get that $\gamma\left(t_{1}\right)-\gamma\left(t_{2}\right)$ is orthogonal to $y-z=\gamma(p)-\gamma(q)$. Hence, applying (3.3) with $\|u\|=1$ and $\theta$ to $\gamma\left(t_{1}\right)-\gamma\left(t_{2}\right)$ and $\gamma(p)-\gamma(q)$, we get

$$
\left(t_{1}-t_{2}\right)(p-q)\left\langle u+\theta w_{t_{1}, t_{2}}, u+\theta w_{p, q}\right\rangle=0
$$

which is impossible as $t_{1} \neq t_{2}, p \neq q$, and $3 \theta \in(0,1)$. Finally, use that for $\tau_{1} \neq \tau_{2}$, the sets $M_{y, z, \tau_{1}} \backslash S_{y, z}$ and $M_{y, z, \tau_{2}} \backslash S_{y, z}$ are disjoint to conclude, as $M_{y, z, \tau} \subseteq I$ for all $\tau$, that there is a countable set $T_{y, z}$ of such $\tau$, satisfying (3.8), for which the Lebesgue measure of $M_{y, z, \tau}$ is positive. Let $T=\bigcup_{(y, z) \in \Gamma} T_{y, z}$. This is a countable set. In addition to (3.8), we now prescribe that $\tau$ lies outside of the countable set $T$. Let $N=N_{f, \varepsilon, I}:=P \cup \bigcup_{(y, z) \in \Gamma} M_{y, z, \tau}$. Then $N$ is a null, closed subset of $I$. Recall that the function $f_{\varepsilon, I}$ is given on $\operatorname{Im}(\gamma)$ by (3.9). By the Intermediate Value Theorem, for any two points $x_{i}=\gamma\left(t_{i}\right) \in \gamma(I)$, $t_{i} \in I, i=1,2$ and $t_{1}<t_{2}$, for which the minimum in the formula (3.9) for $f_{\varepsilon, I}\left(x_{i}\right)$ is attained at different $y=y_{i} \in \gamma(P), i=1,2$, there has to be a point $t_{3} \in\left[t_{1}, t_{2}\right]$ with $t_{3} \in M_{y_{1}, y_{2}, \tau} \subseteq N$. Therefore, the first assertion of (ii) is valid.

For the second assertion of (ii), it remains to note that the set

$$
C=\bigcup_{(y, z) \in \Gamma}\left\{x \in \mathbb{R}^{d}: f(y)+\tau\|x-y\|=f(z)+\tau\|x-z\|\right\}
$$

is closed, and for each open component $J$ of $I \backslash N$, there exists an open component $U_{f}(\gamma(J))$ of $B(\operatorname{Im}(\gamma), \eta) \backslash C$ that contains $\gamma(J)$. Thus, $\left.f_{\varepsilon, I}\right|_{U_{f}(\gamma(J))}=\left.\varphi_{\gamma\left(p_{J}\right)}\right|_{U_{f}(\gamma(J))}$, and (3.7) holds. 
Lemma 3.11. Let $\gamma:[0,1] \rightarrow(0,1)^{d}$ be a 1 -Lipschitz curve that is $\theta$-flat around an interval $R_{0} \subseteq[0,1]$ in direction $u \in \mathbb{S}^{d-1}$, where $\theta \in(0,1 / 3)$. Let $(a, b) \subseteq R_{0}, q \in R_{0} \backslash(a, b)$, and $r \in \mathbb{R}$, $\tau>0$, and let $h:[0,1]^{d} \rightarrow \mathbb{R}$ be a Lipschitz function with

$$
h(x)=r+\tau\|x-\gamma(q)\| \quad \text { for } x \in\{\gamma(a), \gamma(b)\} .
$$

Then

$$
\begin{array}{ll}
|(h \circ \gamma)|_{a}^{b}-\tau(b-a) \mid \leq 3 \theta \tau(b-a) & \text { if } q \leq a \leq b, \text { and } \\
|(h \circ \gamma)|_{a}^{b}-\tau(a-b) \mid \leq 3 \theta \tau(b-a) & \text { if } a \leq b \leq q,
\end{array}
$$

where $\left.(h \circ \gamma)\right|_{a} ^{b}=(h \circ \gamma)(b)-(h \circ \gamma)(a)$.

Proof. In what follows, we adopt the notation of Remark 3.4 and, in particular, make use of the identity (3.3) for points $t_{1}, t_{2} \in R_{0}$. Observe that

$$
\begin{aligned}
\gamma(a)-\gamma(q) & =(a-q) u+\theta(a-q) w_{a, q} \text { and } \\
\gamma(b)-\gamma(q) & =\gamma(a)-\gamma(q)+(b-a) u+\theta(b-a) w_{b, a} \\
& =(b-q) u+\theta(b-q) w_{a, q}+\theta(b-a)\left(w_{b, a}-w_{a, q}\right) .
\end{aligned}
$$

Hence

$$
\begin{aligned}
& \|\gamma(a)-\gamma(q)\|=|a-q|\left\|u+\theta w_{a, q}\right\| \quad \text { and } \\
& |\|\gamma(b)-\gamma(q)\|-| b-q\left|\left\|u+\theta w_{a, q}\right\|\right| \leq 2 \theta(b-a) .
\end{aligned}
$$

Hence, if $q \leq a \leq b$, then

$$
\begin{aligned}
& |(h \circ \gamma)|_{a}^{b}-\tau(b-a) \mid \\
& =\tau|(\|\gamma(b)-\gamma(q)\|-\|\gamma(a)-\gamma(q)\|)-(b-a)| \\
& =\tau\left|\|\gamma(b)-\gamma(q)\|-(a-q)\left\|u+\theta w_{a, q}\right\|-(b-a)\right| \\
& =\tau\left|\|\gamma(b)-\gamma(q)\|-(b-q)\left\|u+\theta w_{a, q}\right\|+(b-a)\left\|u+\theta w_{a, q}\right\|-(b-a)\right| \\
& \leq 2 \theta \tau(b-a)+\tau(b-a)||\left|u+\theta w_{a, q} \|-1\right| \leq 3 \theta \tau(b-a) .
\end{aligned}
$$

If $a \leq b \leq q$, then

$$
\begin{gathered}
|(h \circ \gamma)|_{a}^{b}-\tau(a-b) \mid \\
=\tau|(\|\gamma(b)-\gamma(q)\|-\|\gamma(a)-\gamma(q)\|)-(a-b)| \\
=\tau\left|\|\gamma(b)-\gamma(q)\|-(q-a)\left\|u+\theta w_{a, q}\right\|-(a-b)\right| \\
=\tau\left|\|\gamma(b)-\gamma(q)\|-(q-b)\left\|u+\theta w_{a, q}\right\|+(a-b)\left\|u+\theta w_{a, q}\right\|-(a-b)\right| \\
\leq 2 \theta \tau(b-a)+\tau(b-a)\left|\left\|u+\theta w_{a, q}\right\|-1\right| \leq 3 \theta \tau(b-a) .
\end{gathered}
$$


Lemma 3.12. If $f:[a, b] \rightarrow \mathbb{R}$ is a Lipschitz function; $N \subseteq[a, b]$ is a closed, null set; and $(a, b) \backslash N=$ $\bigcup_{n=1}^{\infty}\left(a_{n}, b_{n}\right)$ is a union of disjoint, open components; then $f(b)-f(a)=\sum_{n \geq 1}\left(f\left(b_{n}\right)-f\left(a_{n}\right)\right)$.

Proof. Observe that

$$
f(b)-f(a)=\int_{a}^{b} f^{\prime}(t) d t=\sum_{n \geq 1} \int_{a_{n}}^{b_{n}} f^{\prime}(t) d t=\sum_{n \geq 1}\left(f\left(b_{n}\right)-f\left(a_{n}\right)\right) .
$$

Lemma 3.13. Let $\gamma:[0,1] \rightarrow(0,1)^{d}$ and $E \subseteq F \subseteq[0,1]$ satisfy the hypotheses of Theorem 2.8, and suppose that $\gamma$ is affine modulo $F$. Suppose $f \in \operatorname{Lip}_{1}\left([0,1]^{d}\right)$ is such that $\operatorname{Lip}(f)<1$. Assume an open set $U \subseteq[0,1]$ such that $U \cap F$ is dense in $F$ is given, $0<\theta^{\prime} \leq \theta<1 / 250^{3}$, and $\varepsilon \in(0,1)$. Suppose $R \subseteq R_{0} \subseteq[0,1]$ are open intervals such that $\gamma$ is $\theta$-flat around $R_{0}$ and $R \cap F \neq \emptyset$. Suppose further that $f_{\varepsilon, R_{0}}$ is a $\theta$-conical function given by Lemma 3.10 .

Then there is an open interval $R_{1} \subseteq R \cap U$ such that $\gamma$ is $\theta^{\prime}$-flat around $R_{1}$, and the following statement holds:

Approximation property 3.13a: Let $g \in \operatorname{Lip}_{1}\left([0,1]^{d}\right)$ with

$$
\operatorname{Lip}(g)<1 \quad \text { and } \quad\left\|g-f_{\varepsilon, R_{0}}\right\|_{\infty}<\theta \mathcal{L}\left(R_{1}\right) / 4,
$$

$\varepsilon^{\prime} \in\left(0, \theta \mathcal{L}\left(R_{1}\right) / 4\right)$ and $g_{\varepsilon^{\prime}, R_{1}}$ be a $\theta^{\prime}$-conical function given by Lemma 3.10. Then there exists an open interval $V$ such that

(i) $\bar{V} \subseteq R_{1} \cdot \mathcal{L}(V) \leq \mathcal{L}\left(R_{1}\right) / 2$, and $V \cap F \neq \emptyset$;

(ii) $g_{\mathcal{E}^{\prime}, R_{1}}$ is continuously differentiable on an open neighbourhood of $\gamma(V)$; for points $x$ from this neighbourhood its gradient $\nabla g_{\varepsilon^{\prime}, R_{1}}(x)$ is given by the formula $(3.7)$ with $\tau \in\left(1-\theta^{\prime}, 1\right)$ and $p \in R_{1}$.

(iii) For every $t \in V$ and $s \in[0,1]$ it holds

$$
\left|\left(f_{\varepsilon, R_{0}}(\gamma(s))-f_{\varepsilon, R_{0}}(\gamma(t))\right)-\left(g_{\mathcal{E}^{\prime}, R_{1}}(\gamma(s))-g_{\mathcal{E}^{\prime}, R_{1}}(\gamma(t))\right)\right| \leq 2 \theta^{1 / 3}|s-t| .
$$

Proof. Consider the closed, null set $N=N_{f, \varepsilon, R_{0}} \subseteq R_{0}$ defined by Lemma 3.10 for the function $f_{\varepsilon, R_{0}}$. Since $\mathcal{L}(N)=0, R \cap F \neq \emptyset$ and $F$ has every portion of positive measure, we have $R \cap F \nsubseteq N$. Hence, we may choose one open component $J_{0}$ of $R_{0} \backslash N$ for which $J_{0} \cap R \cap F \neq \emptyset$. As $U \cap F$ is dense in $F$ and $J_{0} \cap R$ is open, we conclude $U \cap J_{0} \cap R \cap F \neq \emptyset$. Find then an open interval $J^{\prime} \subseteq J_{0} \cap R \cap U$ such that $J^{\prime} \cap F \neq \emptyset$. Apply Lemma 3.8 to get an open interval $J^{\prime \prime} \subseteq J^{\prime}$ such that $\gamma$ is $\theta^{\prime}$-flat around $J^{\prime \prime}$ and $J^{\prime \prime} \cap F \neq \emptyset$. By Remark 3.7, find a $\theta$-density interval $I_{\Delta}\left(t_{0}\right)$ for $F$ such that $I_{\Delta}\left(t_{0}\right) \subseteq J^{\prime \prime}$. Let $R_{1}=I_{\Delta}\left(t_{0}\right)$. Then, using Remark 3.5 for the latter statement, we get that

$$
R_{1} \subseteq J^{\prime \prime} \subseteq J^{\prime} \subseteq J_{0} \cap R \cap U \subseteq R \cap U \quad \text { and } \quad \gamma \text { is } \theta^{\prime} \text {-flat around } R_{1} .
$$

Note that all assertions of the lemma for the interval $R_{1}$, apart from those contained in the Approximation property $3.13 \mathrm{a}$, are already verified. We turn our attention to proving $3.13 \mathrm{a}$ (i)-(iii).

Let $g \in \operatorname{Lip}_{1}\left([0,1]^{d}\right)$ be given according to (3.10), and let

$$
\varepsilon^{\prime} \in\left(0, \theta \mathcal{L}\left(R_{1}\right) / 4\right)
$$

Let $g_{\varepsilon^{\prime}, R_{1}}$ be a $\theta^{\prime}$-conical function given by the hypothesis of $3.13 \mathrm{a}$ and $N^{\prime}=N_{g, \varepsilon^{\prime}, R_{1}}$ be the corresponding closed null set, as given by Lemma 3.10. For brevity, denote $\hat{f}=f_{\varepsilon, R_{0}}$ and $\hat{g}=g_{\varepsilon^{\prime}, R_{1}}$.

As $R_{1} \subseteq J_{0} \subseteq R_{0} \backslash N$, there is, by Lemma 3.10 (ii), a point $p \in N \subseteq R_{0} \backslash R_{1}$ and a constant $\tau \in(1-\theta, 1)$ satisfying the formula $\hat{f}(x)=f(\gamma(p))+\tau\|x-\gamma(p)\|$ for each $x \in \gamma\left(R_{1}\right) \subseteq \gamma\left(J_{0}\right)$. Note that $p \notin R_{1}$ allows us, without loss of generality, to assume that $p$ is to the left of the interval $R_{1}$. Let $U_{f}\left(\gamma\left(J_{0}\right)\right)$ be the open neighbourhood of $\gamma\left(J_{0}\right)$ guaranteed by Lemma 3.10 (ii) such that $\hat{f}$ is continuously differentiable on $U_{f}\left(\gamma\left(J_{0}\right)\right)$. 
Consider all open components $C$ of $R_{1} \backslash N^{\prime}$, and enumerate them as $C_{n}=\left(a_{n}, b_{n}\right)$. We will assume the more complicated case when there are infinitely many such components so that every natural number $n$ is assigned bijectively to a component $C_{n}$. Then Lemma 3.10 (ii) similarly provides $p_{n} \in N^{\prime} \subseteq R_{1}$ and $\tau^{\prime} \in\left(1-\theta^{\prime}, 1\right)$, with respect to which $\hat{g}(x)=g\left(\gamma\left(p_{n}\right)\right)+\tau^{\prime}\left\|x-\gamma\left(p_{n}\right)\right\|$ for all $x \in \gamma\left(C_{n}\right)$. Let $U_{g}\left(\gamma\left(C_{n}\right)\right)$ be the open neighbourhood of $\gamma\left(C_{n}\right)$ such that $\hat{g}$ is continuously differentiable on $U_{g}\left(\gamma\left(C_{n}\right)\right)$.

Hence, for each $n \geq 1$,

$$
W_{n}:=U_{f}\left(\gamma\left(J_{0}\right)\right) \cap U_{g}\left(\gamma\left(C_{n}\right)\right)
$$

is an open neighbourhood of $\gamma\left(C_{n}\right)$ such that both $\left.\hat{f}\right|_{W_{n}}$ and $\left.\hat{g}\right|_{W_{n}}$ are continuously differentiable, so that for every $t \in R_{1} \backslash N^{\prime}$, the gradients $\nabla \hat{f}(\gamma(t))$ and $\nabla \hat{g}(\gamma(t))$ are well-defined. Moreover, for every $n \geq 1$, the functions $\left.\hat{f}\right|_{\gamma\left(C_{n}\right)}$ and $\left.\hat{g}\right|_{\gamma\left(C_{n}\right)}$ satisfy the conditions of Lemma 3.11 for $h$. The only difference will be that for all $n \geq 1$, the functions $\left.\hat{f}\right|_{\gamma\left(C_{n}\right)}$ will use the same $q=p \in N$, whilst the functions $\left.\hat{g}\right|_{\gamma\left(C_{n}\right)}$ may use different $q=p_{n} \in N^{\prime}$. Moreover, by our assumption, we have that $p<a_{n}<b_{n}$ for any $n \geq 1$, but we may have $p_{n}<a_{n}<b_{n}$ for some $n \geq 1$ and $a_{n}<b_{n}<p_{n}$ for others. Let

$$
\begin{array}{ll}
\mathbb{G}=\left\{n \geq 1: p_{n}<a_{n}<b_{n}\right\}, & G=\bigcup_{n \in \mathbb{G}}\left(a_{n}, b_{n}\right) \quad \text { (good sets), } \\
\mathbb{B}=\left\{n \geq 1: a_{n}<b_{n}<p_{n}\right\}, \quad B=\bigcup_{n \in \mathbb{B}}\left(a_{n}, b_{n}\right) \quad \text { (bad sets), }
\end{array}
$$

and note for future reference that $\mathbb{G} \cup \mathbb{B}=\mathbb{N}$ and $G \cup B=\cup_{n \geq 1} C_{n}=R_{1} \backslash N^{\prime}$.

Write $R_{1}=(a, b)$, and denote by $u \in \mathbb{S}^{d-1}$ the vector such that $\gamma$ is $\theta$-flat around $R_{0}$ in direction $u$. Consider the following sets:

$$
\begin{aligned}
X_{0}= & N^{\prime} \cup\{a, b\}, \\
X_{1}= & \left\{t \in \bar{R}_{1} \backslash X_{0}:|\langle\nabla \hat{f}(\gamma(t)), u\rangle-\langle\nabla \hat{g}(\gamma(t)), u\rangle| \geq \theta^{1 / 3}\right\}, \\
X_{2}= & \left\{t \in \bar{R}_{1} \backslash\left(X_{0} \cup X_{1}\right): \exists s \in \bar{R}_{1} \backslash\{t\}\right. \text { such that } \\
& \left.\quad|(\hat{f}(\gamma(s))-\hat{f}(\gamma(t)))-(\hat{g}(\gamma(s))-\hat{g}(\gamma(t)))| \geq 2 \theta^{1 / 3}|s-t|\right\} .
\end{aligned}
$$

We now show that the union $X_{0} \cup X_{1} \cup X_{2}$ is closed. As an intermediate step, we first prove that $X_{0} \cup X_{1}$ is closed. To see this, recall that for each $n \geq 1$, we have that both $\hat{f}$ and $\hat{g}$ are continuously differentiable on $\gamma\left(C_{n}\right) \subseteq U_{f}\left(\gamma\left(J_{0}\right)\right) \cap U_{g}\left(\gamma\left(C_{n}\right)\right)$. Therefore, $X_{1}$ intersects each $C_{n}$ in a relatively closed set: that is, there is a closed set $K_{n} \subseteq \bar{R}_{1}$ such that $X_{1} \cap C_{n}=K_{n} \cap C_{n}$. Hence, $X_{1}=\cup_{n=1}^{\infty}\left(K_{n} \cap C_{n}\right)$. Let $\left(t_{i}\right)_{i=1}^{\infty}$ be a sequence in $X_{1} \cup X_{0}=X_{0} \cup \bigcup_{n=1}^{\infty}\left(K_{n} \cap C_{n}\right)$ such that $t_{i} \rightarrow t \in \bar{R}_{1}$. We need to to verify that $t \in X_{1} \cup X_{0}$. We distinguish two cases: if there exists $n_{0} \in \mathbb{N}$ such that $t \in C_{n_{0}}$, then there is $m_{0} \in \mathbb{N}$ such that $t_{i} \in\left(X_{1} \cup X_{0}\right) \cap C_{n_{0}}=K_{n_{0}} \cap C_{n_{0}}$ for all $i \geq m_{0}$. Since $K_{n_{0}}$ is closed, we conclude that $t=\lim t_{i} \in K_{n_{0}}$. Hence $t \in K_{n_{0}} \cap C_{n_{0}} \subseteq X_{1}$. In the remaining case, we have that $t \in \bar{R}_{1} \backslash \bigcup_{n=1}^{\infty} C_{n} \subseteq X_{0}$.

Now we proceed to show that $X_{0} \cup X_{1} \cup X_{2}$ is closed. Given that $X_{0} \cup X_{1}$ is closed, it suffices to check that the limit of any convergent sequence in $X_{2}$ belongs to $X_{0} \cup X_{1} \cup X_{2}$. Let $\left(t_{i}\right)_{i=1}^{\infty}$ be a convergent sequence in $X_{2}$ with limit $t \in \bar{R}_{1}$. For each $i \in \mathbb{N}$, we may choose $s_{i} \in \bar{R}_{1}$, witnessing that $t_{i} \in X_{2}$; and, by passing to a subsequence if necessary, we may assume that the sequence $\left(s_{i}\right)_{i=1}^{\infty}$ converges to a point $s \in \bar{R}_{1}$. We distinguish two cases: if $s \neq t$, then taking limits as $i \rightarrow \infty$ in (3.15) for $s_{i}$ and $t_{i}$ implies $t \in X_{2}$. Assume now $s=t \notin X_{0}$. Then there exists $n_{0} \geq 1$ such that $s=t \in C_{n_{0}}$, and $t_{i}, s_{i} \in$ $C_{n_{0}} \subseteq R_{1}$ for all $i$ sufficiently large, say $i \geq m_{0}$. Recall that $\gamma$ is $\theta$-flat around $R_{0} \supseteq R_{1}$ in direction $u$. 
Thus,

$$
\begin{aligned}
& \left|\frac{\hat{f}\left(\gamma\left(s_{i}\right)\right)-\hat{f}\left(\gamma\left(t_{i}\right)\right)}{s_{i}-t_{i}}-\frac{\hat{f}\left(\gamma\left(t_{i}\right)+u\left(s_{i}-t_{i}\right)\right)-\hat{f}\left(\gamma\left(t_{i}\right)\right)}{s_{i}-t_{i}}\right| \\
\leq & \operatorname{Lip}(\hat{f})\left|\frac{\gamma\left(s_{i}\right)-\left(\gamma\left(t_{i}\right)+u\left(s_{i}-t_{i}\right)\right)}{s_{i}-t_{i}}\right| \leq \theta
\end{aligned}
$$

and, similarly,

$$
\left|\frac{\hat{g}\left(\gamma\left(s_{i}\right)\right)-\hat{g}\left(\gamma\left(t_{i}\right)\right)}{s_{i}-t_{i}}-\frac{\hat{g}\left(\gamma\left(t_{i}\right)+u\left(s_{i}-t_{i}\right)\right)-\hat{g}\left(\gamma\left(t_{i}\right)\right)}{s_{i}-t_{i}}\right| \leq \theta .
$$

Hence from (3.15), we get, for all $i \geq m_{0}$,

$$
\left|\frac{\left(\hat{f}\left(\gamma\left(t_{i}\right)+u\left(s_{i}-t_{i}\right)\right)-\hat{f}\left(\gamma\left(t_{i}\right)\right)\right)}{s_{i}-t_{i}}-\frac{\left.\left(\hat{g}\left(\gamma\left(t_{i}\right)+u\left(s_{i}-t_{i}\right)\right)\right)-\hat{g}\left(\gamma\left(t_{i}\right)\right)\right)}{s_{i}-t_{i}}\right| \geq 2 \theta^{1 / 3}-2 \theta
$$

For each $i \in \mathbb{N}$, we let

$$
v_{i}(\hat{f}):=\hat{f}\left(\gamma\left(t_{i}\right)+u\left(s_{i}-t_{i}\right)\right)-\hat{f}\left(\gamma\left(t_{i}\right)\right)-\left(s_{i}-t_{i}\right)\left\langle\nabla \hat{f}\left(\gamma\left(t_{i}\right)\right), u\right\rangle
$$

and define $v_{i}(\hat{g})$ similarly. Note that $\lim _{i \rightarrow \infty} \frac{v_{i}(h)}{s_{i}-t_{i}}=0$ for $h=\hat{f}, \hat{g}$. To see this, denote

$$
D_{h}(r, \rho)= \begin{cases}\frac{h(\gamma(r)+\rho u)-h(\gamma(r))}{\rho}-\langle\nabla h(\gamma(r)), u\rangle, & \text { if } \rho \neq 0 \\ 0, & \text { if } \rho=0\end{cases}
$$

for $r \in C_{n_{0}}$ and $\rho \in \mathbb{R}$, where, for the purposes of this formula, we extend the functions $h=\hat{f}, \hat{g}$ arbitrarily outside of $[0,1]^{d}$. We now show that the two functions $D_{\hat{f}}, D_{\hat{g}}: C_{n_{0}} \times \mathbb{R} \rightarrow \mathbb{R}$ are continuous at the points $(r, 0)$. Let $r_{0} \in C_{n_{0}}$; choose positive $\delta_{0}$ and $\rho_{0}$ small enough that $I_{2 \delta_{0}}\left(r_{0}\right) \subseteq C_{n_{0}}$ and $B\left(\gamma\left(I_{\delta_{0}}\left(r_{0}\right)\right), \rho_{0}\right) \subseteq W_{n_{0}}$, where $W_{n_{0}} \supseteq \gamma\left(C_{n_{0}}\right)$ is the open set defined by (3.13) on which both $\hat{f}$ and $\hat{g}$ are continuously differentiable. Then, given $r \in I_{\delta_{0}}\left(r_{0}\right)$ and $|\rho|<\rho_{0}$, we have that the segment $[\gamma(r), \gamma(r)+\rho u]$ is contained in $W_{n_{0}}$. Therefore, $\nabla h$ is well-defined (and continuous) along this segment, and we may apply the Mean Value Theorem to write

$$
D_{h}(r, \rho)=\left\langle\nabla h\left(\gamma(r)+\eta_{\rho} \rho u\right), u\right\rangle-\langle\nabla h(\gamma(r)), u\rangle \quad \text { for some } \eta_{\rho} \in(0,1) .
$$

Since $r \in I_{\delta_{0}}\left(r_{0}\right)$ and $|\rho|<\rho_{0}$ were arbitrary, we may let $r \rightarrow r_{0}$ and $\rho \rightarrow 0$ in the formula above. Using the continuity of $\nabla h$ in $W_{n_{0}}$, we get $\lim _{r \rightarrow r_{0}, \rho \rightarrow 0} D_{h}(r, \rho)=D_{h}\left(r_{0}, 0\right)=0$, verifying the continuity of $D_{h}$ at $\left(r_{0}, 0\right)$ and, in particular, $\frac{v_{i}(\hat{f})}{s_{i}-t_{i}}=D_{\hat{f}}\left(t_{i}, s_{i}-t_{i}\right) \rightarrow 0$ and $\frac{v_{i}(\hat{g})}{s_{i}-t_{i}}=D_{\hat{g}}\left(t_{i}, s_{i}-t_{i}\right) \rightarrow 0$.

After substituting $v_{i}(\hat{f})$ and $v_{i}(\hat{g})$ into (3.16) and choosing $m_{1} \geq m_{0}$ large enough that $\left|\frac{v_{i}(h)}{s_{i}-t_{i}}\right|<\theta / 2$ for both $h=\hat{f}, \hat{g}$ and $i \geq m_{1}$, we derive

$$
\left|\left\langle\nabla \hat{f}\left(\gamma\left(t_{i}\right)\right), u\right\rangle-\left\langle\nabla \hat{g}\left(\gamma\left(t_{i}\right)\right), u\right\rangle\right| \geq 2 \theta^{1 / 3}-2 \theta-\frac{\left|v_{i}(\hat{f})\right|}{\left|s_{i}-t_{i}\right|}-\frac{\left|v_{i}(\hat{g})\right|}{\left|s_{i}-t_{i}\right|} \geq 2 \theta^{1 / 3}-3 \theta>\theta^{1 / 3}
$$

for all $i \geq m_{1}$. Letting $i \rightarrow \infty$ in the above, and using that both $\hat{f}$ and $\hat{g}$ are continuously differentiable on $\gamma\left(C_{n_{0}}\right)$, by Lemma 3.10 (ii), we prove that $t \in X_{1}$. This finishes the proof that $X_{0} \cup X_{1} \cup X_{2}$ is closed.

We will now find an upper bound for the Lebesgue measure of $X_{0} \cup X_{1} \cup X_{2} \subseteq \bar{R}_{1}$, showing that it is much smaller than $\mathcal{L}\left(R_{1}\right)$; see (3.30) for the precise bound. It is clear that $\mathcal{L}\left(X_{0}\right)=0$; let us proceed 
to get estimates of the Lebesgue measure of $X_{1}$ and $X_{2}$. Recall the definition (3.14) of the sets $\mathbb{G}$ and $\mathbb{B}$ and the notation introduced in Lemma 3.11. We assert that

$$
\begin{array}{cc}
|(\hat{f} \circ \gamma-\hat{g} \circ \gamma)|_{a_{n}}^{b_{n}} \leq 7 \theta\left(b_{n}-a_{n}\right), & \text { if } n \in \mathbb{G}, \\
\left.(\hat{f} \circ \gamma-\hat{g} \circ \gamma)\right|_{a_{n}} ^{b_{n}} \geq\left(b_{n}-a_{n}\right), & \text { if } n \in \mathbb{B} .
\end{array}
$$

Indeed, recall that $C_{n}=\left(a_{n}, b_{n}\right)$ is an open component of $R_{1} \backslash N^{\prime} \subseteq R_{0} \backslash N, \gamma$ is $\theta$-flat around $R_{0}$, $p, p_{n} \in R_{0} \backslash C_{n}$, and both $\hat{f}$ and $\hat{g}$ have the special form of Lemma 3.10 (ii) on $\gamma\left(C_{n}\right)$ with respect to the points $p$ and $p_{n}$ and scalars $\tau \in(1-\theta, 1)$ and $\tau^{\prime} \in\left(1-\theta^{\prime}, 1\right)$, respectively. Therefore, we may apply Lemma 3.11 to get

$$
\begin{aligned}
& |(\hat{f} \circ \gamma)|_{a_{n}}^{b_{n}}-\tau\left(b_{n}-a_{n}\right) \mid \leq 3 \theta \tau\left(b_{n}-a_{n}\right) \leq 3 \theta\left(b_{n}-a_{n}\right), \quad n \in \mathbb{N}, \\
& |(\hat{g} \circ \gamma)|_{a_{n}}^{b_{n}}-\tau^{\prime}\left(b_{n}-a_{n}\right) \mid \leq 3 \theta \tau^{\prime}\left(b_{n}-a_{n}\right) \leq 3 \theta\left(b_{n}-a_{n}\right), \quad n \in \mathbb{G}, \\
& |(\hat{g} \circ \gamma)|_{a_{n}}^{b_{n}}-\tau^{\prime}\left(a_{n}-b_{n}\right) \mid \leq 3 \theta \tau^{\prime}\left(b_{n}-a_{n}\right) \leq 3 \theta\left(b_{n}-a_{n}\right), \quad n \in \mathbb{B} .
\end{aligned}
$$

This immediately implies the first inequality of (3.17): as both $\hat{f}$ and $\hat{g}$ are $\theta$-conical, we have $\left|\tau^{\prime}-\tau\right| \leq \theta$ and $\tau^{\prime}+\tau \geq 2-2 \theta$. Hence, for any $n \in \mathbb{G}$

$$
|(\hat{f} \circ \gamma-\hat{g} \circ \gamma)|_{a_{n}}^{b_{n}}|\leq| \tau^{\prime}-\tau \mid\left(b_{n}-a_{n}\right)+6 \theta\left(b_{n}-a_{n}\right) \leq 7 \theta\left(b_{n}-a_{n}\right) .
$$

To see the second inequality of (3.17), we note that if $n \in \mathbb{B}$, then

$$
|(\hat{f} \circ \gamma-\hat{g} \circ \gamma)|_{a_{n}}^{b_{n}}-\left(\tau+\tau^{\prime}\right)\left(b_{n}-a_{n}\right) \mid \leq 6 \theta\left(b_{n}-a_{n}\right) .
$$

Hence $\left.(\hat{f} \circ \gamma-\hat{g} \circ \gamma)\right|_{a_{n}} ^{b_{n}} \geq\left(\tau+\tau^{\prime}-6 \theta\right)\left(b_{n}-a_{n}\right)>b_{n}-a_{n}$, using $\tau^{\prime}+\tau-6 \theta \geq 2-8 \theta$ and $\theta<1 / 10$.

Using Lemma 3.12, $\mathbb{B} \cup \mathbb{G}=\mathbb{N}$, and (3.17), we deduce

$$
\left.(\hat{f} \circ \gamma-\hat{g} \circ \gamma)\right|_{a} ^{b}=\left.\sum_{n \in \mathbb{G}}(\hat{f} \circ \gamma-\hat{g} \circ \gamma)\right|_{a_{n}} ^{b_{n}}+\left.\sum_{n \in \mathbb{B}}(\hat{f} \circ \gamma-\hat{g} \circ \gamma)\right|_{a_{n}} ^{b_{n}} \geq\left.\sum_{n \in \mathbb{G}}(\hat{f} \circ \gamma-\hat{g} \circ \gamma)\right|_{a_{n}} ^{b_{n}}+\mathcal{L}(B),
$$

where $B$ is defined along with $G$ in (3.14). Note that the absolute value of the first summand can be estimated using (3.17) as

$$
\left|\sum_{n \in \mathbb{G}}(\hat{f} \circ \gamma-\hat{g} \circ \gamma)\right|_{a_{n}}^{b_{n}} \mid \leq 7 \theta \sum_{n \in \mathbb{G}}\left(b_{n}-a_{n}\right)=7 \theta \mathcal{L}(G) \leq 7 \theta \mathcal{L}\left(R_{1}\right) .
$$

In addition, using $\hat{g}=g_{\varepsilon^{\prime}, R_{1}}$, Lemma 3.10 (i), (3.10), and (3.12), we get

$$
\|\hat{f}-\hat{g}\|_{\infty} \leq\|\hat{g}-g\|_{\infty}+\|g-\hat{f}\|_{\infty}<\varepsilon^{\prime}+\theta \mathcal{L}\left(R_{1}\right) / 4 \leq \theta \mathcal{L}\left(R_{1}\right) / 2
$$

Hence $|(\hat{f} \circ \gamma-\hat{g} \circ \gamma)|_{a}^{b} \mid \leq \theta \mathcal{L}\left(R_{1}\right)$, and we conclude that

$$
\mathcal{L}(B) \leq \theta \mathcal{L}\left(R_{1}\right)+7 \theta \mathcal{L}\left(R_{1}\right)=8 \theta \mathcal{L}\left(R_{1}\right)
$$

We now show that for $t \in C_{n}$ with $n \in \mathbb{G}$, the gradients $\nabla \hat{f}(\gamma(t))$ and $\nabla \hat{g}(\gamma(t))$ differ in norm by less than the threshold $\theta^{1 / 3}$ defining the set $X_{1}$; see (3.25). This will imply $X_{1} \subseteq B \cup N^{\prime} \cup\{a, b\}$ so that

$$
\mathcal{L}\left(X_{1}\right) \leq \mathcal{L}(B) \leq 8 \theta \mathcal{L}\left(R_{1}\right)<\theta^{1 / 3} \mathcal{L}\left(R_{1}\right) .
$$


Indeed, to estimate the norm of the difference between $\nabla \hat{f}(\gamma(t))$ and $\nabla \hat{g}(\gamma(t))$, we use (3.7) of Lemma 3.10 (ii) to write, for $x=\gamma(t) \in \gamma\left(C_{n}\right)$ and $p^{\prime}=p_{n}$,

$$
\begin{aligned}
\|\nabla \hat{g}(\gamma(t))-\nabla \hat{f}(\gamma(t))\| & =\left\|\tau^{\prime} \frac{x-\gamma\left(p^{\prime}\right)}{\left\|x-\gamma\left(p^{\prime}\right)\right\|}-\tau \frac{x-\gamma(p)}{\|x-\gamma(p)\|}\right\| \\
& \leq\left|\tau^{\prime}-\tau\right|+\tau\left\|\frac{x-\gamma\left(p^{\prime}\right)}{\left\|x-\gamma\left(p^{\prime}\right)\right\|}-\frac{x-\gamma(p)}{\|x-\gamma(p)\|}\right\| \\
& \leq \theta+\left\|\frac{x-\gamma\left(p^{\prime}\right)}{\left\|x-\gamma\left(p^{\prime}\right)\right\|}-\frac{x-\gamma(p)}{\|x-\gamma(p)\|}\right\| .
\end{aligned}
$$

Let

$$
v_{1}=x-\gamma\left(p^{\prime}\right)=\gamma(t)-\gamma\left(p^{\prime}\right) \quad \text { and } \quad v_{2}=x-\gamma(p)=\gamma(t)-\gamma(p)
$$

Note that as $n \in \mathbb{G}$ and $t \in\left(a_{n}, b_{n}\right)$, we have $t>p^{\prime}$. Note also that $p<p^{\prime}$ as $p$ is to the left of $R_{1}$,

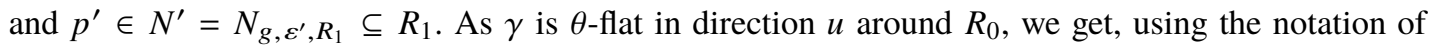
Remark 3.4, for $p<p^{\prime}<t$,

$$
\begin{aligned}
& v_{1}=\left(t-p^{\prime}\right)\left(u+\theta w_{t, p^{\prime}}\right) ; \text { hence }\left\|v_{1}\right\|=\left(t-p^{\prime}\right) q_{t, p^{\prime}} \text { with } q_{t, p^{\prime}} \in(1-\theta, 1+\theta), \\
& v_{2}=(t-p)\left(u+\theta w_{t, p}\right) ; \text { hence }\left\|v_{2}\right\|=(t-p) q_{t, p} \text { with } q_{t, p} \in(1-\theta, 1+\theta) .
\end{aligned}
$$

Therefore, we have

$$
\frac{v_{1}}{\left\|v_{1}\right\|}=\frac{u+\theta w_{t, p^{\prime}}}{q_{t, p^{\prime}}}, \quad \frac{v_{2}}{\left\|v_{2}\right\|}=\frac{u+\theta w_{t, p}}{q_{t, p}} .
$$

Note that both $\frac{1}{q_{t, p}}$ and $\frac{1}{q_{t, p^{\prime}}}$ are at least $\frac{1}{1+\theta} \geq 1-\theta$ and are at most $\frac{1}{1-\theta} \leq 1+2 \theta \leq 2$, as $\theta<1 / 2$. Hence $\left|\frac{1}{q_{t, p^{\prime}}}-\frac{1}{q_{t, p}}\right| \leq 3 \theta$, and their sum is at most 4 , so that

$$
\begin{aligned}
\left\|\frac{v_{1}}{\left\|v_{1}\right\|}-\frac{v_{2}}{\left\|v_{2}\right\|}\right\| & \leq\left\|u\left(\frac{1}{q_{t, p^{\prime}}}-\frac{1}{q_{t, p}}\right)\right\|+\theta\left(\frac{1}{q_{t, p^{\prime}}}+\frac{1}{q_{t, p}}\right) \\
& \leq\left|\frac{1}{q_{t, p^{\prime}}}-\frac{1}{q_{t, p}}\right|+4 \theta \leq 7 \theta .
\end{aligned}
$$

Together with (3.21), this gives

$$
\|\nabla \hat{f}(\gamma(t))-\nabla \hat{g}(\gamma(t))\| \leq \theta+\left\|\frac{v_{1}}{\left\|v_{1}\right\|}-\frac{v_{2}}{\left\|v_{2}\right\|}\right\| \leq 8 \theta<\theta^{1 / 3} .
$$

Having verified the bound (3.20) on the measure of $X_{1}$, we turn our attention to $X_{2}$. Let $\bar{f}(t):=(\hat{f} \circ \gamma)(t)$ and $\bar{g}(t):=(\hat{g} \circ \gamma)(t)$. Then (3.25) and $\operatorname{Lip}(\gamma) \leq 1$ imply

$$
\int_{G}\left|\bar{f}^{\prime}(s)-\bar{g}^{\prime}(s)\right| d s \leq 8 \theta \mathcal{L}(G) \leq 8 \theta(b-a) .
$$

Consider the following variant of the uncentred Hardy-Littlewood maximal function $\mathbb{M} \varphi$ (see [10]), defined for Lebesgue measurable $\varphi: \mathbb{R} \rightarrow \mathbb{R}$ such that $\varphi \in L_{\text {loc }}^{1}(\mathbb{R})$ :

$$
\mathbb{M} \varphi(t)=\sup _{s \in \mathbb{R} \backslash\{t\}} \frac{1}{|s-t|} \int_{[s, t]}|\varphi(r)| d r .
$$


We will use that for any $q>1$, the maximal function satisfies the following inequality, which follows from [10, Theorem 21.76]:

$$
\int_{\mathbb{R}}(\mathbb{M} \varphi(t))^{q} d t \leq 2\left(\frac{q}{q-1}\right)^{q} \int_{\mathbb{R}}|\varphi(t)|^{q} d t .
$$

We will use this inequality with $q=2$ and $\varphi \in L_{\text {loc }}^{1}(\mathbb{R})$ defined by $\varphi:=\left(\bar{f}^{\prime}-\bar{g}^{\prime}\right) \chi_{R_{1}}$, which trivially satisfies

$$
|\varphi(r)| \leq 2 \text { for almost all } r \in \mathbb{R} .
$$

Let $t \in X_{2} \subseteq \bar{R}_{1}$, and choose $s$ according to (3.15). Then $[s, t] \subseteq \bar{R}_{1}$, so that the equality $\left.\varphi\right|_{[s, t]}=$ $\left.\left(\bar{f}^{\prime}-\bar{g}^{\prime}\right)\right|_{[s, t]}$ holds in $L^{1}$, implying

$$
\begin{aligned}
\mathbb{M} \varphi(t) \geq \frac{1}{|s-t|} \int_{[s, t]}|\varphi(r)| d r \geq \frac{1}{|s-t|}\left|\int_{[s, t]} \varphi(r) d r\right| \\
\quad=\frac{1}{|s-t|}\left|\int_{[s, t]}\left(\bar{f}^{\prime}(r)-\bar{g}^{\prime}(r)\right) d r\right|=\frac{|(\bar{f}-\bar{g})|_{s}^{t} \mid}{|s-t|} \geq 2 \theta^{1 / 3},
\end{aligned}
$$

where the last inequality comes from (3.15) for $s$ and $t$. Since $t \in X_{2}$ was arbitrary, we use (3.27) with $q=2$ to derive

$$
\begin{aligned}
\theta^{2 / 3} \mathcal{L}\left(X_{2}\right) & \leq \frac{1}{4} \int_{\mathbb{R}}(\mathbb{M} \varphi(s))^{2} d s \leq 2 \int_{\mathbb{R}}|\varphi(s)|^{2} d s \leq 4 \int_{\mathbb{R}}|\varphi(s)| d s=4 \int_{R_{1}}|\varphi(s)| d s \\
& =4 \int_{B}|\varphi(s)| d s+4 \int_{G}|\varphi(s)| d s \leq 8 \mathcal{L}(B)+32 \theta(b-a) \leq 96 \theta(b-a) .
\end{aligned}
$$

Here we also used (3.28) for all $s \in \mathbb{R}$, followed by (3.26) and (3.19). Hence

$$
\mathcal{L}\left(X_{2}\right) \leq 96 \theta^{1 / 3} \mathcal{L}\left(R_{1}\right) .
$$

Together with (3.20), this implies

$$
\mathcal{L}\left(X_{0} \cup X_{1} \cup X_{2}\right)<100 \theta^{1 / 3} \mathcal{L}\left(R_{1}\right) .
$$

Recall that $R_{1}=I_{\Delta}\left(t_{0}\right)=\left(t_{0}-\Delta, t_{0}+\Delta\right)$ is a $\theta$-density interval for $F$ and that $\theta<1 / 250^{3}$, which implies $1-\theta>240 \theta^{1 / 3}$. Then for $R_{1}^{\prime}=I_{\Delta / 2}\left(t_{0}\right)=\left(t_{0}-\Delta / 2, t_{0}+\Delta / 2\right)$, it holds that the open set $V^{\prime}=R_{1}^{\prime} \backslash\left(X_{0} \cup X_{1} \cup X_{2}\right)$ is of measure bounded below by $\mathcal{L}\left(R_{1}^{\prime}\right)-100 \theta^{1 / 3} \mathcal{L}\left(R_{1}\right)$, whereas $\mathcal{L}\left(R_{1}^{\prime} \cap F\right) \geq(1-\theta) \mathcal{L}\left(R_{1}^{\prime}\right)=\frac{1}{2}(1-\theta) \mathcal{L}\left(R_{1}\right)>100 \theta^{1 / 3} \mathcal{L}\left(R_{1}\right)$. This implies that $V^{\prime} \cap F \neq \emptyset$. Choose an open interval $V$ such that $V \subseteq V^{\prime}$ and $V \cap F \neq \emptyset$. Using that $F$ has every portion of positive measure and $N^{\prime} \subseteq R_{1}$ is a closed set of measure zero, we deduce that there is an open interval $V \subseteq V^{\prime} \backslash N^{\prime}$ with $V \cap F \neq \emptyset$. Part (ii) of the Approximation property 3.13a now follows from $V \subseteq R_{1} \backslash N^{\prime}$ and Lemma 3.10 (ii).

We also have $\bar{V} \subseteq \overline{R_{1}^{\prime}} \subseteq R_{1}$ and $\mathcal{L}(V) \leq \mathcal{L}\left(R_{1}^{\prime}\right)=\mathcal{L}\left(R_{1}\right) / 2$. Now all assertions of part (i) of the Approximation property $3.13 \mathrm{a}$ are established.

To check its remaining part (iii) and (3.11), we can immediately see that for any $t \in V \subseteq R_{1} \backslash\left(X_{0} \cup\right.$ $\left.X_{1} \cup X_{2}\right)$ and $s \in R_{1}$, we have (3.11); see (3.15) and the definition of $X_{2}$. If $t \in V$ and $s \in[0,1] \backslash R_{1}$. Then $t \in V \subseteq R_{1}^{\prime}$ implies $|s-t| \geq \mathcal{L}\left(R_{1}\right) / 4$. Therefore, using $\|\hat{f}-\hat{g}\|_{\infty} \leq \theta \mathcal{L}\left(R_{1}\right) / 2$ from (3.18), we get

$$
|(\hat{f}(\gamma(s))-\hat{f}(\gamma(t)))-(\hat{g}(\gamma(s))-\hat{g}(\gamma(t)))| \leq \theta \mathcal{L}\left(R_{1}\right) \leq 4 \theta|s-t|<2 \theta^{1 / 3}|s-t| .
$$

This proves (3.11) for all $t \in V$ and $s \in[0,1]$, and thus part (iii) of the Approximation property 3.13a. 
We will prove Theorem 2.8 using the Banach-Mazur game. We presently state a short description of the Banach-Mazur game; for more details, see [11].

Definition 3.14. Let $X$ be a non-empty topological space and $S \subseteq X$ its subset, which we refer to as a target set. We define the Banach-Mazur game $G_{B M}(S)$ on $X$ as follows. Players I and II choose alternatively non-empty open sets $G_{i}$ (choices of Player I) and $H_{i}$ (choices of Player II) such that $G_{k} \supseteq H_{k} \supseteq G_{k+1}$ for each $k \geq 1$, and Player II is declared the winner if $\bigcap H_{k} \subseteq S$.

The main result of the Banach-Mazur game that will be useful to us is the following theorem; see [11, Theorem 8.33].

Theorem 3.15. Let $X$ be a non-empty topological space. Then $S \subseteq X$ is residual in $X$ if and only if Player II has a winning strategy in $G_{B M}(S)$.

We may immediately observe that in the case of metric spaces, with topology defined by the metric, we may check the residuality of $S$ in a slightly easier way.

Theorem 3.16. Let $X$ be a non-empty metric space. If Player II has a winning strategy in $G_{B M \text {,balls }}(S)$, the Banach-Mazur game with the restriction that both players may supply only non-empty, open balls as their choices of open sets, then $S$ is residual in $X$.

Proof. We show that Player II has a winning strategy in $G_{B M}(S)$. Assume Player I supplies non-empty open sets $G_{k}$. For each $k \geq 1$, Player II picks $\varphi_{k} \in G_{k}$ and finds $r_{k}>0$ such that $B\left(\varphi_{k}, r_{k}\right) \subseteq G_{k}$, then gives a response $H_{k}=B\left(\psi_{k}, \rho_{k}\right)$, via their strategy in $G_{B M}$, balls to $B\left(\varphi_{k}, r_{k}\right)$. Note that $H_{k}$ is an open set and $H_{k} \subseteq G_{k}$, so the sequence of open sets $\left(G_{k}, H_{k}\right)$ satisfies Definition 3.14. Moreover, since Player II's winning strategy in $G_{B M}$,balls $(S)$ guarantees that $\bigcap H_{k} \subseteq S$, it also provides a winning strategy for Player II in $G_{B M}(S)$. By Theorem 3.15, this implies that $S$ is residual in $X$.

Another simple fact we will need is the following lemma, in which $C^{1}(H)$ denotes the set of continuous functions $\varphi:[0,1]^{d} \rightarrow \mathbb{R}$ for which $\left.\varphi\right|_{\operatorname{Int}(H)}$ is $C^{1}$.

Lemma 3.17. Let $f:[0,1]^{d} \rightarrow \mathbb{R}$ be a Lipschitz function with $\operatorname{Lip}(f) \leq 1$. Then for every $\varepsilon>0$, there exists $g:[0,1]^{d} \rightarrow \mathbb{R}$ such that $\operatorname{Lip}(g)<1$ and $\|f-g\|_{\infty}<\varepsilon$. If, moreover, $f \in C^{1}(H)$ for some $H \subseteq[0,1]^{d}$, then the function $g$ may also be chosen to be in $C^{1}(H)$.

Proof. If $\|f\|_{\infty} \neq 0$, let $g=r f$, with $r \in\left(\max \left(0,1-\frac{\varepsilon}{\|f\|_{\infty}}\right), 1\right)$.

We are now ready to give a proof of Theorem 2.8, the statement of which we repeat here for the reader's convenience.

Theorem 2.8. Let $\emptyset \neq F \subseteq[0,1]$ be a closed set with every portion of positive measure, and let $E$ be a relatively residual subset of $F$. Let $\gamma:[0,1] \rightarrow(0,1)^{d}$ be a Lipschitz curve with Lipschitz constant 1 , such that $\gamma$ is differentiable with derivative of magnitude one at each $t \in E$. Then the set $S$ of those functions $f \in \operatorname{Lip}_{1}\left([0,1]^{d}\right)$ for which there exists $t \in E$ such that $f$ is differentiable with derivative of magnitude one at $\gamma(t)$ is residual in $\operatorname{Lip}_{1}\left([0,1]^{d}\right)$.

Proof. We prove Theorem 2.8 by describing a winning strategy for Player II in the Banach-Mazur game $G_{B M}$,balls $(S)$ in $\operatorname{Lip}_{1}\left([0,1]^{d}\right)$, in which Player I's choices are balls $B\left(\varphi_{k}, r_{k}\right)$ and Player II's choices are balls $B\left(\psi_{k}, \rho_{k}\right)$.

By Lemma 3.2, we may assume that $\gamma$ is affine modulo $F$. Let $(0,1)=U_{0} \supseteq U_{1} \supseteq U_{2} \supseteq \ldots$ be a sequence of open sets such that $U_{n} \cap F$ is dense in $F$ for each $n \geq 1$, and $\left(\cap_{n=0}^{\infty} U_{n}\right) \cap F \subseteq E$. Fix a strictly decreasing sequence of positive numbers $\theta_{k}$ such that $\theta_{1}<1 / 250^{3}$ and $\sum_{k=1}^{\infty} \theta_{k}^{1 / 3}$ converges; for example, let $\theta_{k}=2^{-3 k} / 250^{3}$. For most of the proof, we will only use that $\theta_{k} \downarrow 0$; the convergence property of the series will be used only at the very end of the proof (see (3.36)). In addition to defining $\psi_{k} \in \operatorname{Lip}_{1}\left([0,1]^{d}\right)$ and $\rho_{k}>0$ for each $k \geq 1$, Player II also defines the following additional 
objects: sequences of positive numbers $\varepsilon_{k}$, unit vectors $u_{k}$, open intervals $I_{k}, J_{k} \subseteq[0,1]$, and functions $\varphi_{k}^{(1)} \in \operatorname{Lip}_{1}\left([0,1]^{d}\right)$. These objects have the following properties, for each $k \geq 1$ :

(A) $\operatorname{Lip}\left(\varphi_{k}^{(1)}\right)<1$ and $\varphi_{k}^{(1)} \in B\left(\varphi_{k}, r_{k} / 4\right)$.

(B) $\psi_{k}=\left(\varphi_{k}^{(1)}\right)_{\varepsilon_{k}, J_{k}} \in \operatorname{Lip}_{1}\left([0,1]^{d}\right)$ is a $\theta_{k}$-conical function given by Lemma 3.10.

(C) (i) $I_{k} \subseteq J_{k} \subseteq I_{k-1} \cap U_{k-1}$.

(ii) $\bar{I}_{k} \subseteq I_{k-1}$, and $\mathcal{L}\left(I_{k}\right) \leq \mathcal{L}\left(I_{k-1}\right) / 2$.

(iii) $I_{k} \cap F \neq \emptyset$.

(iv) $\gamma$ is $\theta_{k}$-flat in direction $u_{k}$ around both $J_{k}$ and $I_{k}$.

(D) For $k \geq 2$, the function $\psi_{k}$ is continuously differentiable on an open neighbourhood of $\gamma\left(I_{k}\right)$; for points $x$ from this neighbourhood, its gradient $\nabla \psi_{k}(x)$ is given by the right-hand side of (3.7) with $\tau>1-\theta_{k}$ and $p \in J_{k}$.

(E) For $k \geq 2$,

$$
\left|\left(\psi_{k}(\gamma(s))-\psi_{k}(\gamma(t))\right)-\left(\psi_{k-1}(\gamma(s))-\psi_{k-1}(\gamma(t))\right)\right| \leq 2 \theta_{k-1}^{1 / 3}|s-t|
$$

for all $t \in I_{k}$ and $s \in[0,1]$.

(F)

(i) $\varepsilon_{k} \in\left(0, \min \left\{\frac{r_{k}}{2}, \frac{\theta_{k} \mathcal{L}\left(J_{k}\right)}{4}\right\}\right)$.

(ii) For $k \geq 2, \rho_{k-1}<\theta_{k-1} \mathcal{L}\left(J_{k}\right) / 4$, and $\bar{B}\left(\psi_{k-1}, \rho_{k-1}\right) \subseteq B\left(\varphi_{k-1}, r_{k-1}\right)$.

Consider Player I's first move $B\left(\varphi_{1}, r_{1}\right)$. Use Lemma 3.17 to find $\varphi_{1}^{(1)} \in B\left(\varphi_{1}, r_{1} / 4\right)$ such that $\operatorname{Lip}\left(\varphi_{1}^{(1)}\right)<1$; this establishes (A) for $k=1$. Apply Lemma 3.8 with $\theta=\theta_{1}$ to find an open interval $J_{1} \subseteq[0,1]$ and $u_{1} \in \mathbb{S}^{d-1}$ such that $\gamma$ is $\theta_{1}$-flat in direction $u_{1}$ around $J_{1}$ and $J_{1} \cap F \neq \emptyset$. Let $\varepsilon_{1}$ be chosen arbitrarily subject to (Fi) for $k=1$, and let $\psi_{1}:=\left(\varphi_{1}^{(1)}\right)_{\varepsilon_{1}, J_{1}}$ be a $\theta_{1}$-conical function given by Lemma 3.10, verifying (B) for $k=1$. We declare $\psi_{1}$ as the first function played by Player II.

Let $I_{1} \subseteq J_{1}$ be an open interval such that $\bar{I}_{1} \subseteq(0,1), I_{1} \cap F \neq \emptyset$, and $\mathcal{L}\left(I_{1}\right) \leq 1 / 2$. Setting $I_{0}=(0,1)$, we see that all parts of (C) are satisfied with $k=1$.

We have thus verified all properties (A)-(F) for $k=1$, including (D), (E), and (Fii), for which there is nothing to verify in the case $k=1$.

Let $n \geq 2$. On Step $n$, Player II does the following main actions:

- Defines $\rho_{n-1}$ so that (Fii) is satisfied with $k=n$.

- Accepts Player I's choice of $\left(\varphi_{n}, r_{n}\right)$ such that $B\left(\varphi_{n}, r_{n}\right) \subseteq B\left(\psi_{n-1}, \rho_{n-1}\right)$.

- Defines $\psi_{n} \in B\left(\varphi_{n}, r_{n}\right)$.

Let $f:=\varphi_{n-1}^{(1)}, U:=U_{n-1}, \theta:=\theta_{n-1}, \theta^{\prime}:=\theta_{n}, \varepsilon:=\varepsilon_{n-1}, R:=I_{n-1}, R_{0}:=J_{n-1}$, and $f_{\varepsilon, R_{0}}:=\psi_{n-1}$.

These objects satisfy the conditions of Lemma 3.13, namely

- $\operatorname{Lip}(f)<1$, by (A) for $k=n-1$.

- $R \subseteq R_{0}$, by (Ci) for $k=n-1$.

- $R \cap F \neq \emptyset$, by (Ciii) for $k=n-1$.

- $\gamma$ is $\theta$-flat around $R_{0}$, by (Civ) for $k=n-1$.

- $f_{\varepsilon, R_{0}}$ is a $\theta$-conical function given by Lemma 3.10, due to (B) with $k=n-1$.

Let

$$
J_{n}:=R_{1} \subseteq R \cap U=I_{n-1} \cap U_{n-1}
$$

be the open interval given by Lemma 3.13 applied with these settings. This verifies the second inclusion of (Ci) with $k=n$.

From (B) with $k=n-1$ and Lemma 3.10 (i), it follows that $\left\|\psi_{n-1}-\varphi_{n-1}^{(1)}\right\|_{\infty}<\varepsilon_{n-1}$. Therefore, by (A) and (Fi) with $k=n-1$, we have $\psi_{n-1} \in B\left(\varphi_{n-1}, r_{n-1}\right)$. Define now a positive number $\rho_{n-1}$ arbitrarily so as to establish (Fii) with $k=n$. 
Assume Player I's $n$th move is an open ball $B\left(\varphi_{n}, r_{n}\right) \subseteq B\left(\psi_{n-1}, \rho_{n-1}\right)$, and make a choice of $\varepsilon_{n}$ and $\varphi_{n}^{(1)} \in \operatorname{Lip}_{1}\left([0,1]^{d}\right)$ verifying $(\mathrm{Fi})$ and $(\mathrm{A})$ for $k=n$, using Lemma 3.17 for the second choice. We declare $\psi_{n}$, defined according to (B) for $k=n$, as the $n$th function of Player II.

We are now ready to apply the Approximation property $3.13 \mathrm{a}$ of $f_{\varepsilon, R_{0}}$. Let $g:=\varphi_{n}^{(1)}, \varepsilon^{\prime}:=\varepsilon_{n}$, and $g_{\varepsilon^{\prime}, R_{1}}:=\psi_{n}$. These objects fit the framework of Lemma 3.13 and satisfy the hypotheses of the Approximation property 3.13a, namely

- $\operatorname{Lip}(g)=\operatorname{Lip}\left(\varphi_{n}^{(1)}\right)<1$ by (A) for $k=n$,

- $\left\|g-f_{\varepsilon, R_{0}}\right\|_{\infty}=\left\|\varphi_{n}^{(1)}-\psi_{n-1}\right\|_{\infty}<\rho_{n-1}<\theta_{n-1} \mathcal{L}\left(J_{n}\right) / 4=\theta \mathcal{L}\left(R_{1}\right) / 4$,

which derives from $\varphi_{n}^{(1)} \in B\left(\varphi_{n}, r_{n}\right) \subseteq B\left(\psi_{n-1}, \rho_{n-1}\right)$, and (Fii) with $k=n$,

- $\varepsilon^{\prime} \in\left(0, \theta \mathcal{L}\left(R_{1}\right) / 4\right)$, due to $(\mathrm{Fi})$ for $k=n$, and

- $g_{\varepsilon^{\prime}, R_{1}}=\psi_{n}$ is a $\theta^{\prime}$-conical function given by Lemma 3.10.

Let

$$
I_{n}:=V \subseteq J_{n}
$$

be the open interval given by the Approximation property 3.13a, applied with the settings above. We then have that (Civ), (Ciii), (D), the remaining inclusion of (Ci), (Cii), and (E) are satisfied with $k=n$.

This verifies all properties (A)-(F) for $k=n$.

Note that (Fii) implies that $\bar{B}\left(\psi_{n}, \rho_{n}\right) \subseteq B\left(\varphi_{n}, r_{n}\right) \subseteq B\left(\psi_{n-1}, \rho_{n-1}\right)$ for each $n \geq 2$, whilst (Fii) and $\theta_{n} \rightarrow 0$ imply $\rho_{n} \rightarrow 0$ as $n \rightarrow \infty$. Hence the intersection of balls $B\left(\psi_{n}, \rho_{n}\right)$ is a single function

$$
f \in \bigcap_{n=1}^{\infty} B\left(\psi_{n}, \rho_{n}\right) \subseteq \operatorname{Lip}_{1}\left([0,1]^{d}\right)
$$

From (Cii), we derive that the intersection of all $I_{n}$ is a single point $t^{*} \in \bigcap_{n=1}^{\infty} I_{n} \subseteq[0,1]$. Moreover, from (Ciii) and (Cii), it follows that $t^{*}$ is a limit point of $F$ and so $t^{*} \in F$. By (Ci), we have $t^{*} \in I_{n} \subseteq U_{n-1}$ for all $n$. Therefore, $t^{*} \in F \cap \bigcap_{n=1}^{\infty} U_{n} \subseteq E$, implying that $\gamma^{\prime}\left(t^{*}\right)$ exists and $\left\|\gamma^{\prime}\left(t^{*}\right)\right\|=1$.

We now show that $f$ is differentiable at $\gamma\left(t^{*}\right)$ in the direction of $\gamma^{\prime}\left(t^{*}\right)$, and this directional derivative is equal to 1 or -1 . Since $f$ is 1-Lipschitz, this will imply that $f$ is (fully) differentiable at $\gamma\left(t^{*}\right)$ (see [9, Corollary 2.6]) and $\left\|\nabla f\left(\gamma\left(t^{*}\right)\right)\right\|=1$.

Let $\varepsilon \in(0,1 / 5)$. Consider any $n \geq 1$. From (Civ), we find a sufficiently small $\delta_{n}>0$ such that for all $s \in\left(I_{n} \backslash\left\{t^{*}\right\}\right) \cap\left(t^{*}-\delta_{n}, t^{*}+\delta_{n}\right)$, it holds

$$
\left\|u_{n}-\gamma^{\prime}\left(t^{*}\right)\right\| \leq \frac{\left\|\gamma(s)-\gamma\left(t^{*}\right)-\left(s-t^{*}\right) u_{n}\right\|+\left\|\gamma(s)-\gamma\left(t^{*}\right)-\left(s-t^{*}\right) \gamma^{\prime}\left(t^{*}\right)\right\|}{\left|s-t^{*}\right|} \leq 2 \theta_{n} .
$$

Notice that the left- and right-hand sides of the above do not depend on $s$. Hence, $\theta_{n} \rightarrow 0$ implies $u_{n} \rightarrow \gamma^{\prime}\left(t^{*}\right)$.

By (D), we have that $\psi_{n}$ is continuously differentiable on an open neighbourhood of $\gamma\left(t^{*}\right)$ with $\nabla \psi_{n}\left(\gamma\left(t^{*}\right)\right)$ given by (3.7) with $\tau=\tau_{n}>1-\theta_{n}$ and $p=p_{n} \in J_{n}$. Thus, there is a $\delta_{n}^{\prime}>0$ such that if $0<\left|s-t^{*}\right|<\delta_{n}^{\prime}$, then

$$
\begin{aligned}
& \left|\frac{\psi_{n}\left(\gamma\left(t^{*}\right)+\left(s-t^{*}\right) u_{n}\right)-\psi_{n}\left(\gamma\left(t^{*}\right)\right)}{s-t^{*}}-\left\langle\nabla \psi_{n}\left(\gamma\left(t^{*}\right)\right), u_{n}\right\rangle\right| \\
= & \left|\frac{\left.\psi_{n}\left(\gamma\left(t^{*}\right)+\left(s-t^{*}\right) u_{n}\right)\right)-\psi_{n}\left(\gamma\left(t^{*}\right)\right)}{s-t^{*}}-\tau_{n}\left\langle\frac{\gamma\left(t^{*}\right)-\gamma\left(p_{n}\right)}{\left\|\gamma\left(t^{*}\right)-\gamma\left(p_{n}\right)\right\|}, u_{n}\right\rangle\right|<\varepsilon / 2 .
\end{aligned}
$$


Also, using (Civ), we get for all $s \in I_{n} \backslash\left\{t^{*}\right\}$,

$$
\left|\frac{\psi_{n}(\gamma(s))-\psi_{n}\left(\gamma\left(t^{*}\right)+\left(s-t^{*}\right) u_{n}\right)}{s-t^{*}}\right| \leq \operatorname{Lip}\left(\psi_{n}\right) \theta_{n} \leq \theta_{n} .
$$

Let $n_{1}>1$ be such that $\theta_{n_{1}}<\varepsilon / 4$ and let $n \geq n_{1}$. Then

$$
\left|\frac{\psi_{n}(\gamma(s))-\psi_{n}\left(\gamma\left(t^{*}\right)\right)}{s-t^{*}}-\tau_{n}\left\langle\frac{\gamma\left(t^{*}\right)-\gamma\left(p_{n}\right)}{\left\|\gamma\left(t^{*}\right)-\gamma\left(p_{n}\right)\right\|}, u_{n}\right\rangle\right|<\varepsilon
$$

for $s \in\left(t^{*}-\delta_{n}^{\prime}, t^{*}+\delta_{n}^{\prime}\right) \cap I_{n} \backslash\left\{t^{*}\right\}$ and $\tau_{n}, p_{n}$, as above.

Recall that $u_{n} \rightarrow \gamma^{\prime}\left(t^{*}\right)$ by (3.33) and $\tau_{n} \geq 1-\theta_{n}$, so $\tau_{n} \rightarrow 1$. Note also that $p_{n}, t^{*} \in J_{n} \subseteq I_{n-1}$ for every $n$, by (Ci), and $\mathcal{L}\left(I_{n}\right) \rightarrow 0$ from (Cii). This implies $\left|p_{n}-t^{*}\right| \rightarrow 0$, and we deduce that

$$
\lim _{n \rightarrow \infty}\left|\tau_{n}\left\langle\frac{\gamma\left(t^{*}\right)-\gamma\left(p_{n}\right)}{\left\|\gamma\left(t^{*}\right)-\gamma\left(p_{n}\right)\right\|}, u_{n}\right\rangle\right|=\left\langle\gamma^{\prime}\left(t^{*}\right), \gamma^{\prime}\left(t^{*}\right)\right\rangle=\left\|\gamma^{\prime}\left(t^{*}\right)\right\|^{2}=1 .
$$

Thus, there is $n_{2} \geq n_{1}$ such that for each $n \geq n_{2}$, there is $\sigma_{n} \in\{-1,+1\}$ with

$$
\left|\tau_{n}\left\langle\frac{\gamma\left(t^{*}\right)-\gamma\left(p_{n}\right)}{\left\|\gamma\left(t^{*}\right)-\gamma\left(p_{n}\right)\right\|}, u_{n}\right\rangle-\sigma_{n}\right|<\varepsilon
$$

However, (E) and (3.34) imply that for $n>n_{2}$,

$$
\left|\tau_{n-1}\left\langle\frac{\gamma\left(t^{*}\right)-\gamma\left(p_{n-1}\right)}{\left\|\gamma\left(t^{*}\right)-\gamma\left(p_{n-1}\right)\right\|}, u_{n-1}\right\rangle-\tau_{n}\left\langle\frac{\gamma\left(t^{*}\right)-\gamma\left(p_{n}\right)}{\left\|\gamma\left(t^{*}\right)-\gamma\left(p_{n}\right)\right\|}, u_{n}\right\rangle\right|<2 \varepsilon+2 \theta_{n-1}^{1 / 3} .
$$

Therefore, choosing $n_{3}>n_{2}$ so that $\theta_{n_{3}}<1 / 1000$, we get that for all $n>n_{3}$,

$$
\left|\sigma_{n-1}-\sigma_{n}\right| \leq 4 \varepsilon+2 \theta_{n-1}^{1 / 3}<1
$$

Hence the sign $\sigma_{n}$ of $\tau_{n}\left\langle\frac{\gamma\left(t^{*}\right)-\gamma\left(p_{n}\right)}{\left\|\gamma\left(t^{*}\right)-\gamma\left(p_{n}\right)\right\|}, u_{n}\right\rangle$ does not change for $n>n_{3}$, and so

$$
\lim _{n \rightarrow \infty} \tau_{n}\left\langle\frac{\gamma\left(t^{*}\right)-\gamma\left(p_{n}\right)}{\left\|\gamma\left(t^{*}\right)-\gamma\left(p_{n}\right)\right\|}, u_{n}\right\rangle
$$

exists and is equal to 1 or -1 . Assume, without loss of generality, that

$$
\lim _{n \rightarrow \infty} \tau_{n}\left\langle\frac{\gamma\left(t^{*}\right)-\gamma\left(p_{n}\right)}{\left\|\gamma\left(t^{*}\right)-\gamma\left(p_{n}\right)\right\|}, u_{n}\right\rangle=1,
$$

and choose $n_{4}>n_{3}$ so that

$$
\left|\tau_{n}\left\langle\frac{\gamma\left(t^{*}\right)-\gamma\left(p_{n}\right)}{\left\|\gamma\left(t^{*}\right)-\gamma\left(p_{n}\right)\right\|}, u_{n}\right\rangle-1\right|<\varepsilon
$$

for all $n \geq n_{4}$, and $\sum_{n=n_{4}}^{\infty} \theta_{n}^{1 / 3}<\varepsilon / 2$. Let $n \geq n_{4}$ and $s \in[0,1] \backslash\left\{t^{*}\right\}$. We claim that

$$
\alpha_{n}:=\left|\frac{f(\gamma(s))-f\left(\gamma\left(t^{*}\right)\right)}{s-t^{*}}-\frac{\psi_{n}(\gamma(s))-\psi_{n}\left(\gamma\left(t^{*}\right)\right)}{s-t^{*}}\right|<\varepsilon .
$$


Indeed, using $\left\|\psi_{k}-f\right\|_{\infty} \rightarrow 0,(\mathrm{E})$, and (Fi), we get

$$
\alpha_{n} \leq \sum_{k=n}^{\infty}\left|\frac{\psi_{k+1}(\gamma(s))-\psi_{k+1}\left(\gamma\left(t^{*}\right)\right)}{s-t^{*}}-\frac{\psi_{k}(\gamma(s))-\psi_{k}\left(\gamma\left(t^{*}\right)\right)}{s-t^{*}}\right| \leq \sum_{k=n}^{\infty} 2 \theta_{k}^{1 / 3}<\varepsilon .
$$

Hence, whenever $0<\left|s-t^{*}\right|<\delta_{n}^{\prime}$, we have, using (3.34), (3.35), and (3.36), that

$$
\left|\frac{f(\gamma(s))-f\left(\gamma\left(t^{*}\right)\right)}{s-t^{*}}-1\right|<3 \varepsilon .
$$

Thus, $f \circ \gamma$ is differentiable at $t^{*}$ with $(f \circ \gamma)^{\prime}\left(t^{*}\right)=1$. Since $\gamma$ is also differentiable with derivative of magnitude one at $t^{*} \in E$, it follows that $f$ is differentiable with derivative of magnitude one at $\gamma\left(t^{*}\right)$; thus $f \in S$, so Player II wins.

Remark 3.18. The proof of Theorem 2.8 given above may be slightly modified to obtain a proof of the stronger statement referred to in Remark 2.9: namely, that in the setting of Theorem 2.8, a typical function $f \in \operatorname{Lip}_{1}\left([0,1]^{d}\right)$ is differentiable with derivative of magnitude one at $\gamma(t)$ for typical $t \in F$. We describe the necessary additional details:

Firstly, we modify the proof of Theorem 2.8 to show that the set of pairs

$$
S_{\times}=\left\{(f, t): f \in \operatorname{Lip}_{1}\left([0,1]^{d}\right), t \in F, f \text { is differentiable at } \gamma(t),\|\nabla f(\gamma(t))\|=1\right\}
$$

is residual in $\operatorname{Lip}_{1}\left([0,1]^{d}\right) \times F$. For this, we define the Banach-Mazur game in $\operatorname{Lip}_{1}\left([0,1]^{d}\right) \times F$, where on each turn, each of the two players supplies a direct product of an open ball around a 1-Lipschitz function and an open interval with non-empty intersection with $F$. Assuming that Player I supplies $B\left(f_{n}, r_{n}\right) \times G_{n}$ on their $n$th turn, define $J_{n} \subseteq I_{n-1} \cap U_{n-1} \cap G_{n}$ (compare with (3.31)). Then the reply $B\left(\psi_{n}, \rho_{n}\right) \times I_{n}$ from Player II will guarantee that Player II wins the game in $\operatorname{Lip}_{1}\left([0,1]^{d}\right) \times F$ with target $S_{\times}$(here, $I_{n}$ is defined by (3.32)).

Having established that $S_{\times}$is residual in $\operatorname{Lip}_{1}\left([0,1]^{d}\right) \times F$, by Theorem 3.15, it only remains to apply the Kuratowski-Ulam theorem (see, for example, [11, Theorem 8.41 (iii)]). As $E$ is a relatively residual subset of $F$, a typical function $f \in \operatorname{Lip}_{1}\left([0,1]^{d}\right)$ is differentiable with derivative of magnitude one at $\gamma(t)$ for typical $t \in E$.

\section{Curve detection of non-coverable sets}

In this section, we prove Theorem 2.11.

Notation and convention. We introduce some notation designed for $L^{\infty}$ mappings $\varphi: I \rightarrow \mathbb{R}^{d}$, where $I \subseteq \mathbb{R}$ is a closed interval. In what follows, $\varphi$ will be either a $C^{1}$-smooth or a Lipschitz mapping or the derivative of such. We use the notation $I(\varphi)$ to denote its domain $I$ and $\operatorname{Im}(\varphi)$ to denote the set of all its values, $\operatorname{Im}(\varphi)=\varphi(I(\varphi))=\varphi(I)$.

For a subset $U \subseteq I$, we consider the quantity

$$
\operatorname{osc}_{\varphi}(U):=\operatorname{ess} \sup \{\|\varphi(s)-\varphi(t)\|: s, t \in U\}
$$

which corresponds to the oscillation of $\varphi$ on the set $U$.

Recall that we call a Lipschitz or a $C^{1}$-smooth mapping $\gamma: I \rightarrow \mathbb{R}^{d}$ a curve if the magnitude of its derivative is bounded away from zero almost everywhere. Moreover, given a $C^{1}$-smooth mapping $\gamma: I \rightarrow \mathbb{R}^{d}$ defined on a closed interval $I$, we interpret the derivative $\gamma^{\prime}$ at the endpoints of $I$ as the one-sided derivative so that $\gamma^{\prime}$ is a well-defined mapping $I \rightarrow \mathbb{R}^{d}$.

Given sets $\mathscr{F}, U \subseteq \mathbb{R}^{d}$ with $U$ open, we define $\Gamma_{\mathscr{F}}(U)$ as the collection of all $C^{1}$-smooth curves $\gamma: I \rightarrow \mathbb{R}^{d}$ with $\mathcal{L}\left(\gamma^{-1}(\mathscr{F})\right)>0$ and $\operatorname{Im}(\gamma) \subseteq U$. 
We let $\theta: \mathbb{R}^{d} \backslash\{0\} \rightarrow \mathbb{S}^{d-1}$ denote standard spherical projection

$$
\theta(x)=\frac{x}{\|x\|}, \quad x \in \mathbb{R}^{d} \backslash\{0\} .
$$

Definition 4.1. For each $n \geq 1$, consider the set $D_{n}$ of $(n-1)$-tuples $\beta=\left(i_{1}, \ldots, i_{n-1}\right)$, where each $i_{j} \in \mathbb{N}$ satisfies $1 \leq i_{j} \leq 2^{d}$. The set $D_{1}$ should be interpreted as a singleton set containing the empty sequence $\emptyset$. For $\beta=\left(i_{1}, \ldots, i_{n-1}\right) \in D_{n}$, let $|\beta|=n$; and for each $m \leq n$, let $\left.\beta\right|_{m}=\left(i_{1}, \ldots, i_{m-1}\right) \in D_{m}$. Define the order on each $D_{n}$ according to the lexicographical order, and extend this to an ordering on $\bigcup_{n=1}^{\infty} D_{n}$ via the following rule: if $\left|\beta^{\prime}\right|<|\beta|$, then let $\beta^{\prime}<\beta$ if $\beta^{\prime} \leq\left.\beta\right|_{\left|\beta^{\prime}\right|}$ and $\beta^{\prime}>\beta$ otherwise.

For each $n \geq 1$, let $\left\{Q_{\beta}\right\}_{\beta \in D_{n}}$ be the standard dyadic partition of $[-1,1]^{d}$ into $2^{(n-1) d}$ closed cubes with side $2^{-n+2}$, such that $Q_{\beta} \subseteq Q_{\beta^{\prime}}$ iff $\left|\beta^{\prime}\right| \leq|\beta|$ and $\left.\beta\right|_{\left|\beta^{\prime}\right|}=\beta^{\prime}$. For each $n \geq 1$ and $\beta \in D_{n}$, let $S_{\beta}=Q_{\beta} \cap \mathbb{S}^{d-1}$. Define

$$
T_{n}=\left\{\beta \in D_{n}: \text { Int } S_{\beta} \neq \emptyset\right\}
$$

where the interior is taken with respect to the subspace topology on $\mathbb{S}^{d-1}$. Note that for any $\beta \in T_{n}$, we have

$$
S_{\beta}=\bigcup\left\{S_{\beta^{\prime}}: \beta^{\prime} \in T_{n+1},\left.\beta^{\prime}\right|_{n}=\beta\right\},
$$

and for any $n \geq 1$,

$$
\mathbb{S}^{d-1}=\bigcup_{\beta \in T_{n}} S_{\beta}
$$

In particular, note that $\mathbb{S}^{d-1}=S_{\emptyset}=\bigcup_{\beta \in T_{1}} S_{\beta}$. For each $\rho>0, n \geq 1$, and $\beta \in T_{n}$, we will also denote by $B\left(S_{\beta}, \rho\right)$ the open $\rho$-neighbourhood of $S_{\beta}$, considered as a subset of $\mathbb{S}^{d-1}$, with respect to the induced topology and Euclidean metric $\|\cdot-\cdot\|_{2}$ from $\mathbb{R}^{d}$.

For $1 \leq m \leq n$ and $\beta^{\prime} \in T_{m}, \beta \in T_{n}$, we let

$$
\zeta\left(\beta^{\prime}, \beta\right)=\max \left\{\|x-y\|_{2}: x \in S_{\beta^{\prime}}, y \in S_{\beta}\right\} .
$$

In this way, $\zeta(\beta, \beta)$ is the Euclidean diameter of $S_{\beta}$. We note for future reference that $\zeta(\beta, \beta) \rightarrow 0$ as $|\beta| \rightarrow \infty$.

For each $k \in \mathbb{N}$, we let $\mathcal{P}_{k}$ denote the collection of open intervals in $[0,1]$ with consecutive $(k-1)$ th level dyadic endpoints. That is,

$$
\mathcal{P}_{k}:=\left\{\left(\frac{i-1}{2^{k-1}}, \frac{i}{2^{k-1}}\right): i=1,2, \ldots, 2^{k-1}\right\} .
$$

Further, we let $\mathcal{D}_{k}$ denote the set of $(k-1)$ th-level dyadic numbers in $[0,1]$; that is,

$$
\mathcal{D}_{k}:=\left\{\frac{i}{2^{k-1}}: i=0,1,2, \ldots, 2^{k-1}\right\}=\bigcup_{I \in \mathcal{P}_{k}} \partial I .
$$

Finally, for a subset $Y$ of $[0,1]$, we will use the notation $Y^{\text {comp }}$ to denote its complement, $[0,1] \backslash Y$.

For the reader's convenience, we repeat the statement to be proved:

Theorem 2.11. Let $d \geq 1$ and $\mathscr{F} \subseteq[0,1]^{d}$ be a non-empty, closed set having every portion of positive cone width. Let $\mathscr{A} \subseteq(0,1)^{d}$ be an analytic set such that $\mathscr{A} \cap \mathscr{F}$ is relatively residual in $\mathscr{F}$. Then there exists a 1-Lipschitz curve $\gamma:[0,1] \rightarrow(0,1)^{d}$ and sets $E \subseteq F \subseteq[0,1]$ with the following properties:

(i) $F$ is non-empty and closed and has every portion of positive measure.

(ii) $E$ is residual in $F$. 
(iii) $\gamma$ is differentiable at every point $t \in E$ with $\left\|\gamma^{\prime}(t)\right\|=1$.

(iv) For every $t \in E$, we have

$$
\lim _{\delta \rightarrow 0} \operatorname{osc}_{\gamma^{\prime}}([t-\delta, t+\delta])=0 .
$$

(v) $\gamma(E) \subseteq \mathscr{A}$.

The proof of Theorem 2.11 occupies the entire remainder of the present section and contains several lemmata, the hypotheses of which should be understood as the current setting in which the statement appears in the proof. Thus, each such statement refers to objects previously constructed.

By hypothesis, there are open sets $O_{1}, O_{2}, \ldots \subseteq \mathbb{R}^{d}$ such that for each $n \in \mathbb{N}$, the set $O_{n} \cap \mathscr{F}$ is a dense subset of $\mathscr{F}$ and

$$
\mathscr{F} \cap \bigcap_{n=1}^{\infty} O_{n} \subseteq \mathscr{A}
$$

We may assume that $O_{1}=\mathbb{R}^{d}$ and $O_{n+1} \subseteq O_{n}$ for all $n \geq 1$.

Iterative construction. Let $L_{1}=c_{1}=1$ and

$$
L_{k}=L_{k-1}+2^{-k}, \quad c_{k}=c_{k-1}-2^{-k}, \quad k \geq 2 .
$$

Remark 4.2. Note that $1 \leq L_{k} \leq 2$ and $1 / 2 \leq c_{k} \leq 1$ for all $k \in \mathbb{N}$. In fact, we could have chosen any strictly decreasing sequence $c_{n}$ and strictly increasing sequence $L_{n}$ with $0<c_{\infty}=\inf _{n \geq 1} c_{n}<c_{1}=$ $1=L_{1}<\sup _{n \geq 1} L_{n}=L_{\infty}<\infty$. This would have changed constants in estimates for derivatives of $\gamma_{k}$ in (A) and (H) below, and hence also in estimates for derivatives of the limit curve $\gamma_{\infty}$; see Lemma 4.3. However, a particular choice of $c_{\infty}$ and $L_{\infty}$ does not affect the strength of the result we prove.

Below, we construct sequences of

- Piecewise $C^{1}$-smooth, Lipschitz curves $\gamma_{k}:[0,1] \rightarrow \mathbb{R}^{d}$,

- Numbers $\alpha_{k}, \lambda_{k}, r_{k}, \rho_{k}, \psi_{k}>0, p_{k} \in \mathbb{N}$,

- Sets

$$
G_{k}=\bigcup_{j=1}^{p_{k}} G_{k, j} \subseteq[0,1], \quad H_{k}=\bigcup_{j=1}^{p_{k}} H_{k, j} \subseteq[0,1]
$$

as finite unions of closed intervals $G_{k, j}$ and $H_{k, j}$,

- Open sets

$$
U_{k}=\bigcup_{j=1}^{p_{k}} U_{k, j} \subseteq(0,1)^{d}
$$

as finite unions of open sets $U_{k, j}$,

- Sets $M_{k}, W_{k} \subseteq[0,1]$ with $M_{k}$ finite and $W_{k} \supseteq M_{k}$ being a finite union of closed intervals,

- Functions $\beta_{k}: H_{k} \rightarrow T_{k}$,

such that the following conditions are satisfied for each $k \geq 1$ :

(A) $\frac{1}{2} \leq c_{k} \leq\left\|\gamma_{k}^{\prime}(t)\right\| \leq L_{k} \leq 2$ for all $t \in[0,1] \backslash M_{k}$.

(B) For any interval $I \in \mathcal{P}_{k}$, either

$$
\mathcal{L}\left(I \cap \gamma_{k}^{-1}\left(\mathscr{F} \cap O_{k}\right) \cap \bigcap_{i=1}^{k-1} W_{i}^{\mathrm{comp}}\right) \geq \alpha_{k} \quad \text { or } \quad I \cap \gamma_{k}^{-1}(\mathscr{F}) \cap \bigcap_{i=1}^{k-1} W_{i}^{\mathrm{comp}}=\emptyset .
$$

(C) If $k \geq 2, I \in \mathcal{P}_{k}$, and $I \cap \gamma_{k-1}^{-1}(\mathscr{F}) \cap \bigcap_{i=1}^{k-1} W_{i}^{\text {comp }}=\emptyset$, then $\gamma_{k}(t)=\gamma_{k-1}(t)$ for all $t \in I$. 
(D) If $k \geq 2$, then

(i) $\left\|\gamma_{k}(t)-\gamma_{k-1}(t)\right\| \leq \psi_{k-1} / 2$ for all $t \in[0,1]$, and

(ii) $\psi_{k} \in\left(0, \psi_{k-1} / 2\right)$.

(E) If $k \geq 2$, then

(i) $\mathcal{L}\left(\left\{t \in[0,1]: \gamma_{k}(t) \neq \gamma_{k-1}(t)\right\}\right)<\frac{\alpha_{k-1}}{4}$, and

(ii) $0<\alpha_{k} \leq 2^{-k} \alpha_{k-1}$.

(F) $H_{k}$ is the union of finitely many pairwise disjoint, closed intervals $H_{k, j}, j=1, \ldots, p_{k}$. These sets have the following properties:

(i) If $k \geq 2, I \in \mathcal{P}_{k}$, and $\gamma_{k-1}^{-1}(\mathscr{F}) \cap I \cap \bigcap_{i=1}^{k-1} W_{i}^{\text {comp }} \neq \emptyset$, then there exists an index $j \in$ $\left\{1, \ldots, p_{k}\right\}$ such that $H_{k, j} \subseteq I$.

(ii) $H_{k} \subseteq \bigcap_{i=1}^{k-1} W_{i}^{\text {comp }}$ and $\mathcal{L}\left(H_{k, j} \cap \gamma_{k}^{-1}\left(\mathscr{F} \cap O_{k}\right)\right) \geq \alpha_{k}$ for each $j=1, \ldots, p_{k}$.

(iii) For all $1 \leq l<k$, the components of $H_{l}$ and $H_{k}$ are either nested or disjoint. More precisely, for all $l \in\{1, \ldots, k-1\}, j \in\left\{1, \ldots, p_{k}\right\}$, and $i \in\left\{1, \ldots, p_{l}\right\}$, we have

$$
H_{l, i} \cap H_{k, j}=\emptyset \quad \text { or } \quad H_{k, j} \subseteq \operatorname{Int}\left(H_{l, i}\right) .
$$

(iv) $\bar{B}\left(\gamma_{k}\left(H_{k, j}\right), \psi_{k}\right) \subseteq U_{k, j} \subseteq O_{k}$ for all $j \in\left\{1, \ldots, p_{k}\right\}$.

(v) $\left.\beta_{k}\right|_{H_{k, j}} \in T_{k}$ is constant with value

$$
\beta_{k, j}:=\min \left\{\beta \in T_{k}: \exists \gamma \in \Gamma_{\mathscr{F}}\left(U_{k, j}\right) \text { s.t. } \operatorname{Im}\left(\theta\left(\gamma^{\prime}\right)\right) \subset B\left(S_{\beta}, 2^{-k}\right)\right\}
$$

(vi) If $t \in H_{k} \cap H_{l}$ with $1 \leq l<k$, then $\beta_{k}(t)>\beta_{l}(t)$.

(G) (Throw away sets.)

(i) $M_{k}=M_{k-1} \cup \mathcal{D}_{k} \cup \bigcup_{j=1}^{p_{k}}\left(\partial H_{k, j} \cup \partial G_{k, j}\right) \cup \bigcup_{i=1}^{k-1} \partial W_{i}$ is a finite set, and the restriction of $\gamma_{k}$ to each component of $[0,1] \backslash M_{k}$ is $C^{1}$-smooth.

(ii) $W_{k}$ is a finite union of closed sub-intervals of $[0,1]$, $M_{k} \subseteq$ Int $W_{k} \cup\{0,1\} \subseteq W_{k}$, and $\mathcal{L}\left(W_{k}\right) \leq 2^{-k} \alpha_{k}$.

(H) (Convergence of derivatives.)

(i) If $t \in[0,1] \backslash\left(H_{k} \cup M_{k}\right)$, then $\left\|\gamma_{k}^{\prime}(t)-\gamma_{k-1}^{\prime}(t)\right\| \leq 2^{-k}$.

(ii) If $k \geq 2$ and $t \in H_{k} \backslash M_{k}$, then $\left\|\left|\gamma_{k}^{\prime}(t)\|-\| \gamma_{k-1}^{\prime}(t) \|\right| \leq 2^{-k}\right.$.

(iii) The mapping $t \mapsto\left\|\gamma_{k}^{\prime}(t)\right\|$ is constant on each component of $[0,1] \backslash M_{k}$.

(iv) If $t \in H_{k} \backslash M_{k}$, then $\theta\left(\gamma_{k}^{\prime}(t)\right) \in B\left(S_{\beta_{k}(t)}, 2^{-k}\right)$.

Let $M_{0}=W_{0}=\emptyset$. Use Remark 1.2 to find a $C^{1}$-smooth curve $\gamma_{1}:[0,1] \rightarrow(0,1)^{d}$ with $\left\|\gamma_{1}^{\prime}(t)\right\|=1$ for all $t \in[0,1]$ and

$$
\alpha_{1}:=\mathcal{L}\left(\gamma_{1}^{-1}(\mathscr{F})\right)>0
$$

Choose $\psi_{1}>0$ small enough that $\bar{B}\left(\operatorname{Im}\left(\gamma_{1}\right), \psi_{1}\right) \subseteq(0,1)^{d}$. Further, set

$$
\begin{array}{lrl}
p_{1}=r_{1}=\rho_{1}=\lambda_{1}=1, \quad M_{1}=\{0,1\}, & W_{1}=\left[0, \frac{\alpha_{1}}{4}\right] \cup\left[1-\frac{\alpha_{1}}{4}, 1\right], \\
G_{1}=G_{1,1}=H_{1}=H_{1,1}=[0,1], & \text { and } & U_{1}=U_{1,1}=(0,1)^{d} \subseteq O_{1} .
\end{array}
$$

Define $\beta_{1}: H_{1} \rightarrow T_{1}=\{\emptyset\}$ as the (only possible) constant function, and set $\beta_{1,1}=\emptyset \in T_{1}$. Then for $k=1$, all conditions $(\mathrm{A})-(\mathrm{H})$ are either trivially satisfied or void.

Assume now that $n \geq 2$ and the conditions (A)-(H) are satisfied for $k=1, \ldots, n-1$. The $n$th step of the construction proceeds as follows. Indeed, let $I_{n, 1}, \ldots, I_{n, p_{n}}$ be an enumeration of those intervals $I \in \mathcal{P}_{n}$ for which $\gamma_{n-1}^{-1}(\mathscr{F}) \cap I \cap \bigcap_{i=1}^{n-1} W_{i}^{\text {comp }} \neq \emptyset$. For each $j=1, \ldots, p_{n}$, we nominate a point $t_{n, j} \in \gamma_{n-1}^{-1}(\mathscr{F}) \cap I_{n, j} \cap \bigcap_{i=1}^{n-1} W_{i}^{\text {comp }}$. As $t_{n, j} \in I_{n, j} \cap \bigcap_{i=1}^{n-1} W_{i}^{\text {comp }}$, and the latter is an open set, we may 
choose $\lambda_{n}>0$ sufficiently small that for all $j=1, \ldots, p_{n}$,

$$
G_{n, j}:=\left[t_{n, j}-\lambda_{n}, t_{n, j}+\lambda_{n}\right] \subseteq I_{n, j} \cap \bigcap_{i=1}^{n-1} W_{i}^{\mathrm{comp}} \quad \text { and } \quad \operatorname{osc}_{\gamma_{n-1}^{\prime}}\left(G_{n, j}\right) \leq 2^{-(n+1)}
$$

The second condition of (4.6) can be achieved due to the fact, coming from (Gi) for $k=n-1$, that $\gamma_{n-1}$ restricted to each component of $[0,1] \backslash M_{n-1}$ is $C^{1}$-smooth. We also impose a further condition on $\lambda_{n}$, as follows:

$$
\lambda_{n} \in\left(0, \frac{1}{8} \min \left(\alpha_{n-1} / p_{n}, \psi_{n-1}\right)\right)
$$

Observe that

$$
\gamma_{n-1}\left(H_{l, i}\right) \subseteq U_{l, i} \quad \text { whenever } l \in\{1, \ldots, n-1\} \text { and } i \in\left\{1, \ldots, p_{l}\right\} .
$$

If $n=2$, this is clear. For $n>2$, we argue as follows. Given indices $l \in\{1, \ldots, n-2\}, i \in\left\{1, \ldots, p_{l}\right\}$, and $t \in H_{l, i}$, we may use (Di) for $l+1 \leq k \leq n-1$ and $\psi_{k} \in\left(0, \psi_{k-1} / 2\right)$ from (Fiv) to deduce that

$$
\left\|\gamma_{n-1}(t)-\gamma_{l}(t)\right\| \leq \sum_{k=l+1}^{n-1} \frac{\psi_{k-1}}{2}<\psi_{l}
$$

In case $l=n-1$, the above inequality is trivially satisfied. Together with (Fiv) for $k \leq n-1$, this verifies (4.8). Now, let $r_{n}>0$ be chosen sufficiently small that

$$
B\left(\gamma_{n-1}\left(H_{l, i}\right), r_{n}\right) \subseteq U_{l, i} \quad \text { whenever } l \in\{1, \ldots, n-1\} \text { and } i \in\left\{1, \ldots, p_{l}\right\}
$$

and

$$
r_{n} \in\left(0,2^{-(n+3)} \lambda_{n}\right)
$$

For each $j=1, \ldots, p_{n}$, we set

$$
U_{n, j}:=B\left(\gamma_{n-1}\left(t_{n, j}\right), r_{n}\right) \cap O_{n}
$$

Note that $U_{n, j}$ is open and has a non-empty intersection with $\mathscr{F}$ due to the density of $\mathscr{F} \cap O_{n}$ in $\mathscr{F}$, and $\gamma_{n-1}\left(t_{n, j}\right) \in \mathscr{F}$. Let

$$
\beta_{n, j}:=\min \left\{\beta \in T_{n}: \exists \gamma \in \Gamma_{\mathscr{F}}\left(U_{n, j}\right) \text { s.t. } \operatorname{Im}\left(\theta\left(\gamma^{\prime}\right)\right) \subset B\left(S_{\beta}, 2^{-n}\right)\right\} .
$$

The hypothesis that $\mathscr{F}$ has every portion of positive cone width guarantees that the set for which the minimum in the definition of $\beta_{n, j}$ is considered is non-empty. For each $j=1, \ldots, p_{n}$, we choose, using Remark 1.2, a $C^{1}$-curve $v_{n, j} \in \Gamma_{\mathscr{F}}\left(U_{n, j}\right)$ such that

$$
\operatorname{Im}\left(\theta\left(v_{n, j}^{\prime}\right)\right) \subseteq B\left(S_{\beta_{n, j}}, 2^{-n}\right) \quad \text { and } \quad\left\|v_{n, j}^{\prime}(t)\right\|=\left\|\gamma_{n-1}^{\prime}\left(t_{n, j}\right)\right\|
$$

for all $t \in I\left(v_{n, j}\right)$. By choosing $\rho_{n}>0$ sufficiently small, in particular

$$
\rho_{n} \in\left(0,2^{-(n+4)} \lambda_{n}\right)
$$

and restricting each $v_{n, j}$ to a smaller and shifted interval and reparameterising if necessary, we may assume that for $j=1, \ldots, p_{n}$, each $v_{n, j}$ is defined on the interval

$$
I\left(v_{n, j}\right)=H_{n, j}:=\left[t_{n, j}-\rho_{n}, t_{n, j}+\rho_{n}\right] \subseteq \operatorname{Int}\left(G_{n, j}\right) .
$$


Note, for future reference, that for each $j=1, \ldots, p_{n}$,

$$
\mathcal{L}\left(v_{n, j}^{-1}\left(\mathscr{F} \cap O_{n}\right)\right)>0 .
$$

We now verify properties (A)-(H) for $k=n$. We start by checking various parts of (F). By definition of $t_{n, j}$ and $H_{n, j}$, we have that (Fi) with $k=n$ is satisfied. Moreover, (Fiii) with $k=n$ is readily verified. We note that $H_{n, j}$ is a subset of a connected component of $\bigcap_{i=1}^{n-1} W_{i}^{\text {comp }} \subseteq[0,1] \backslash M_{n-1}$, whereas $\partial H_{l, i} \subseteq M_{l} \subseteq M_{n-1}$ by (Gi) with $k \leq n-1$. Thus, it is clear that if $H_{l, i} \cap H_{n, j} \neq \emptyset$, then $\operatorname{Int}\left(H_{l, i}\right) \supseteq H_{n, j}$, establishing (Fiii) with $k=n$.

Let $G_{n}$ and $H_{n}$ be defined according to (4.4). Then the first condition of (Fii) with $k=n$ is satisfied. Define $\beta_{n}: H_{n} \rightarrow T_{n}$ by

$$
\beta_{n}(t)=\beta_{n, j}, \quad t \in H_{n, j},
$$

in accordance with $(\mathrm{Fv}), k=n$.

We are now ready to verify (Fvi) with $k=n$. Suppose $t \in H_{n, j} \cap H_{l, i} \neq \emptyset$ for some $l \in\{1, \ldots, n-1\}$, $j \in\left\{1, \ldots, p_{n}\right\}$, and $i \in\left\{1, \ldots, p_{l}\right\}$. Then $H_{n, j} \subseteq H_{l, i}$ by (Fiii), which we already verified for $k=n$. In particular, we have $t_{n, j} \in H_{l, i}$, and therefore $U_{n, j} \subseteq B\left(\gamma_{n-1}\left(t_{n, j}\right), r_{n}\right) \subseteq U_{l, i}$ by (4.11) and (4.9). This trivially implies

$$
\Gamma_{\mathscr{F}}\left(U_{n, j}\right) \subseteq \Gamma_{\mathscr{F}}\left(U_{l, i}\right) .
$$

We will use this inclusion together with the following basic facts, readily verifiable from Definition 4.1:

$$
\begin{aligned}
B\left(S_{\beta}, 2^{-n}\right) & \subseteq B\left(S_{\left.\beta\right|_{l}}, 2^{-l}\right), & & \beta \in T_{n}, \\
\beta & >\left.\beta\right|_{l}, & & \beta \in T_{n},
\end{aligned}
$$

$\left\{\left.\beta\right|_{l}: \beta \in T_{n}\right\}=T_{l}$.

With these properties at hand, together with (4.12) and (4.16), we observe

$$
\begin{aligned}
\beta_{n}(t)=\beta_{n, j} & =\min \left\{\beta \in T_{n},: \exists \gamma \in \Gamma_{\mathscr{F}}\left(U_{n, j}\right) \text { s.t. } \operatorname{Im}\left(\theta\left(\gamma^{\prime}\right)\right) \subset B\left(S_{\beta}, 2^{-n}\right)\right\} \\
& \geq \min \left\{\beta \in T_{n}: \exists \gamma \in \Gamma_{\mathscr{F}}\left(U_{l, i}\right) \text { s.t. } \operatorname{Im}\left(\theta\left(\gamma^{\prime}\right)\right) \subset B\left(S_{\left.\beta\right|_{l}}, 2^{-l}\right)\right\} \\
& >\min \left\{\left.\beta\right|_{l}: \beta \in T_{n}, \exists \gamma \in \Gamma_{\mathscr{F}}\left(U_{l, i}\right) \text { s.t. } \operatorname{Im}\left(\theta\left(\gamma^{\prime}\right)\right) \subset B\left(S_{\left.\beta\right|_{l}}, 2^{-l}\right)\right\} \\
& =\min \left\{\beta \in T_{l}: \exists \gamma \in \Gamma_{\mathscr{F}}\left(U_{l, i}\right) \text { s.t. } \operatorname{Im}\left(\theta\left(\gamma^{\prime}\right)\right) \subset B\left(S_{\beta}, 2^{-l}\right)\right\}=\beta_{l, i}=\beta_{l}(t) .
\end{aligned}
$$

The first inequality above follows from (4.17) and (4.18), the second from (4.19), and the subsequent equality from (4.20). This completes the verification of (Fvi).

We define the new curve $\gamma_{n}:[0,1] \rightarrow \mathbb{R}^{d}$ by

$$
\gamma_{n}(t)= \begin{cases}\gamma_{n-1}(t) & \text { if } t \in[0,1] \backslash \bigcup_{j=1}^{p_{n}} \operatorname{Int}\left(G_{n, j}\right), \\ v_{n, j}(t) & \text { if } t \in H_{n, j}, j=1, \ldots, p_{n},\end{cases}
$$

and the condition that on each of the components of $\bigcup_{j=1}^{p_{n}}\left(G_{n, j} \backslash H_{n, j}\right)$, the curve $\gamma_{n}$ is affine, and hence $\left\|\gamma_{n}^{\prime}(t)\right\|$ is constant. Condition (C) with $k=n$ is clearly satisfied.

Since, for each $j=1, \ldots, p_{n}, \gamma_{n}\left(H_{n, j}\right)=v_{n, j}\left(H_{n, j}\right)$ is a compact subset of the open set $U_{n, j} \subseteq O_{n}$, we may choose $\psi_{n} \in\left(0, \psi_{n-1} / 2\right)$, establishing (Dii) and (Fiv) for $k=n$.

Note that

$$
\left\{t \in[0,1]: \gamma_{n}(t) \neq \gamma_{n-1}(t)\right\} \subseteq \bigcup_{j=1}^{p_{n}} \operatorname{Int}\left(G_{n, j}\right),
$$

and the latter set has measure precisely $2 p_{n} \lambda_{n}$. Therefore, we get (Ei) with $k=n$ by (4.7). From the fact (4.22) that $\gamma_{n}$ and $\gamma_{n-1}$ differ only on the pairwise disjoint intervals $G_{n, j}$ of length $2 \lambda_{n}$, it also follows, 
using again (4.7) and (A), that

$$
\left\|\gamma_{n}-\gamma_{n-1}\right\|_{\infty} \leq\left(L_{n}+L_{n-1}\right) \lambda_{n}<4 \lambda_{n} \leq \psi_{n-1} / 2 .
$$

This verifies (Di) with $k=n$.

Recall (4.15) and (4.21), and set

$$
\alpha_{n}:=\min \left\{2^{-n} \alpha_{n-1}, \min _{1 \leq j \leq p_{n}} \mathcal{L}\left(v_{n, j}^{-1}(\mathscr{F})\right)\right\}>0,
$$

to obtain the remaining part of (Fii), and (Eii) for $k=n$. In particular, all parts of (F) are now established. From (Fii) for $k=n$, the choice of $I_{n, j} \supseteq G_{n, j} \supseteq H_{n, j}$, and (4.22), we derive (B) for $k=n$.

Define $M_{n}$ as in (Gi) with $k=n$. Then we see that the second condition of (Gi) with $k=n$ is satisfied, using (Gi) for $k=n-1,(4.22)$, and the way that $\left.\gamma\right|_{G_{n, j}}$ is defined for each $j \in\left\{1, \ldots, p_{n}\right\}$. For each point in $M_{n}$, we now nominate a small, relatively open interval around this point so that the total measure of the union of all such intervals is at most $2^{-n} \alpha_{n}$. We define $W_{n}$ as the union of closures of these intervals so that (Gii) with $k=n$ is satisfied.

The conditions (Hii), (Hiii), and (Hiv) are now easily verified via (4.21) and (4.13). For (Hii), we additionally use $t_{n, j} \in H_{n, j} \subseteq G_{n, j}$ and (4.6), whilst for (Hiii), we additionally recall (Gi) with $k=n$, (Hiii) for $k=n-1$, and (4.22).

If $t \in[0,1] \backslash\left(M_{n} \cup G_{n}\right)$, then by (4.22) and (Gi) for $k=n$, we have $\gamma_{n}(t)=\gamma_{n-1}(t)$ and $\gamma_{n}^{\prime}(t)=\gamma_{n-1}^{\prime}(t)$. Therefore, both (A) and (Hi) are satisfied for $t$. If $t \in G_{n} \backslash\left(M_{n} \cup H_{n}\right)$, then without loss of generality, $t$ belongs to an interval of the form $\left[t_{n, j}-\lambda_{n}, t_{n, j}-\rho_{n}\right]$, restricted to which $\gamma_{n}$ is affine. Hence,

$$
\begin{aligned}
\gamma_{n}^{\prime}(t) & =\frac{\gamma_{n}\left(t_{n, j}-\rho_{n}\right)-\gamma_{n}\left(t_{n, j}-\lambda_{n}\right)}{\lambda_{n}-\rho_{n}} \\
& =\frac{\gamma_{n-1}\left(t_{n, j}-\rho_{n}\right)-\gamma_{n-1}\left(t_{n, j}-\lambda_{n}\right)}{\lambda_{n}-\rho_{n}}+\frac{\nu_{n, j}\left(t_{n, j}-\rho_{n}\right)-\gamma_{n-1}\left(t_{n, j}-\rho_{n}\right)}{\lambda_{n}-\rho_{n}} .
\end{aligned}
$$

Further, since $\operatorname{Im}\left(v_{n, j}\right) \subseteq U_{n, j} \subseteq B\left(\gamma_{n-1}\left(t_{n, j}\right), r_{n}\right)$, by (4.11), we have

$$
\begin{aligned}
\left\|v_{n, j}\left(t_{n, j}-\rho_{n}\right)-\gamma_{n-1}\left(t_{n, j}-\rho_{n}\right)\right\| & \leq r_{n}+\left\|\gamma_{n-1}\left(t_{n, j}\right)-\gamma_{n-1}\left(t_{n, j}-\rho_{n}\right)\right\| \\
& \leq r_{n}+L_{n-1} \rho_{n} \leq r_{n}+2 \rho_{n} .
\end{aligned}
$$

We conclude that

$$
\left\|\gamma_{n}^{\prime}(t)-\gamma_{n-1}^{\prime}(t)\right\| \leq \operatorname{osc}_{\gamma_{n-1}^{\prime}}\left(G_{n, j}\right)+\frac{r_{n}+2 \rho_{n}}{\lambda_{n}-\rho_{n}} \leq 2^{-(n+1)}+2 \frac{r_{n}+2 \rho_{n}}{\lambda_{n}} \leq 2^{-n},
$$

using (4.6), (4.10), and (4.14). This verifies (Hi) for $k=n$. Moreover, (4.23), (A) for $k=n-1$, and (4.3) imply (A) for $t$ and $k=n$. To complete the verification of (A), note that for $t \in H_{n} \backslash M_{n}$, we can find $j \in\left\{1, \ldots, p_{n}\right\}$ such that $t \in H_{n, j}$, implying $\left\|\gamma_{n}^{\prime}(t)\right\|=\left\|v_{n, j}^{\prime}(t)\right\|=\left\|\gamma_{n-1}^{\prime}\left(t_{n, j}\right)\right\|$, and finally apply (A) for $k=n-1$. Thus, all conditions (A)-(H) hold for the objects of step $k=n$.

The limit curve $\gamma_{\infty}$. By (D), the sequence of mappings $\left(\gamma_{k}\right)_{k=1}^{\infty}$ converges in the supremum norm to a mapping $\gamma_{\infty}:[0,1] \rightarrow \mathbb{R}^{d}$.

Lemma 4.3. The limit curve $\gamma_{\infty}$ has the following properties:

(i) The mapping $\gamma_{\infty}$ is Lipschitz with $\operatorname{Lip}\left(\gamma_{\infty}\right) \leq 2$.

(ii) The mapping $\gamma_{\infty}$ may be viewed as a mapping $[0,1] \rightarrow(0,1)^{d}$, that is, with codomain $(0,1)^{d}$.

(iii) For almost every $t \in[0,1]$, all mappings $\gamma_{k}$ with $k \in \mathbb{N} \cup\{\infty\}$ are differentiable at $t$, and there exists $m=m(t) \in \mathbb{N}$ such that $\gamma_{\infty}^{\prime}(t)=\gamma_{k}^{\prime}(t)$ for all $k \geq m$. 
(iv) For almost every $t \in[0,1], \gamma_{\infty}$ is differentiable at $t$ with $\left\|\gamma_{\infty}^{\prime}(t)\right\| \geq 1 / 2$. Consequently $\gamma_{\infty}$ is a Lipschitz curve.

Proof. Part (i) is trivial, since $\gamma_{\infty}$ is the uniform limit of mappings $\gamma_{k}$, all of which satisfy $\operatorname{Lip}\left(\gamma_{k}\right) \leq 2$, by (A). For (ii), observe that (D) implies

$$
\left\|\gamma_{\infty}-\gamma_{1}\right\|_{\infty} \leq \sum_{k=1}^{\infty} \frac{\psi_{k}}{2}<\psi_{1}
$$

Recall that $\psi_{1}>0$ was chosen sufficiently small that $\bar{B}\left(\operatorname{Im}\left(\gamma_{1}\right), \psi_{1}\right) \subseteq(0,1)^{d}$. We conclude that $\operatorname{Im}\left(\gamma_{\infty}\right) \subseteq(0,1)^{d}$; that is, we may view $\gamma_{\infty}$ as a mapping $[0,1] \rightarrow(0,1)^{d}$. Moving on to (iii), we use (E) to infer

$$
\mathcal{L}\left(\bigcup_{n=m}^{\infty} B_{n}\right) \leq \sum_{n=m}^{\infty} \frac{\alpha_{n}}{4} \leq \frac{\alpha_{m}}{2}, \quad \text { with } B_{n}:=\left\{t \in[0,1]: \gamma_{\infty}(t) \neq \gamma_{n}(t)\right\},
$$

for all $m \geq 1$. Letting $C_{m}:=\bigcap_{n=m}^{\infty} B_{n}^{\text {comp }} \subseteq[0,1]$, we conclude that $\bigcup_{m=1}^{\infty} C_{m}$ has full measure in $[0,1]$. Moreover, for each $m \geq 1$ and almost every density point $t$ of $C_{m}$, we have that all mappings $\gamma_{k}$ with $k \in \mathbb{N} \cup\{\infty\}$ are differentiable at $t$ and $\gamma_{\infty}^{\prime}(t)=\gamma_{n}^{\prime}(t)$ for all $n \geq m$. The statement of (iii) follows. Finally, note that part (iv) follows immediately from (iii) and (A).

Let

$$
F_{\infty}:=\gamma_{\infty}^{-1}(\mathscr{F}) \cap \bigcap_{i=1}^{\infty} W_{i}^{\mathrm{comp}}, \quad H:=\bigcap_{n=1}^{\infty} \bigcup_{k=n}^{\infty} \operatorname{Int}\left(H_{k}\right) .
$$

Lemma 4.4. The sets $F_{\infty}$ and $H$ have the following properties:

(i) The set $H$ is $G_{\delta}$.

(ii) The derivative $\gamma_{k}^{\prime}(t)$ exists for every $t \in H$ and every $k \in \mathbb{N}$.

(iii) The set $F_{\infty}$ is closed.

(iv) $H \cap F_{\infty} \subseteq \gamma_{\infty}^{-1}(\mathscr{A})$.

(v) The set $F_{\infty} \subseteq[0,1]$ is non-empty and has every portion of positive Lebesgue measure.

(vi) For every $k \in \mathbb{N}$ and every component $H_{k, j}$ of $H_{k}$, we have

$$
\operatorname{Int}\left(H_{k, j}\right) \cap F_{\infty} \neq \emptyset \text {. }
$$

(vii) The set $H \cap F_{\infty}$ is a relatively residual subset of $F_{\infty}$.

Proof. The assertion (i) for $H$ is obvious, and the existence of $\gamma_{k}^{\prime}(t)$ in (ii) follows from (Gi), as $H \cap \bigcup_{i=1}^{\infty} M_{i} \neq \emptyset$ by (G) and (Fii). To see that $F_{\infty}$ is a closed subset of $[0,1]$, we argue that $\bigcup_{i=1}^{\infty} W_{i}$ is a relatively open subset of $[0,1]$. Indeed, by (G), we have that $\partial W_{i} \subseteq M_{i+1} \subseteq \operatorname{Int} W_{i+1} \cup\{0,1\}$ for each $i \geq 1$. Hence, as $0,1 \in W_{1}$,

$$
\{0,1\} \cup \bigcup_{i=1}^{\infty} \operatorname{Int} W_{i} \subseteq \bigcup_{i=1}^{\infty} W_{i} \subseteq\{0,1\} \cup \bigcup_{i=1}^{\infty} \operatorname{Int} W_{i} .
$$

It remains to note that

$$
\{0,1\} \cup \bigcup_{i=1}^{\infty} \operatorname{Int} W_{i}=\left(\{0,1\} \cup \operatorname{Int} W_{1}\right) \cup \bigcup_{i=2}^{\infty} \operatorname{Int} W_{i}
$$

is a union of relatively open sets in $[0,1]$. This proves (iii).

For (iv), it suffices to show that $H \cap F_{\infty} \subseteq \gamma_{\infty}^{-1}\left(\cap_{n=1}^{\infty} O_{n} \cap \mathscr{F}\right)$. Fix $t \in H \cap F_{\infty}$ and $n \in \mathbb{N}$. Since $t \in F_{\infty}$, we have $\gamma_{\infty}(t) \in \mathscr{F}$. Since $t \in H$, we may choose $k \geq n$ such that $t \in \operatorname{Int}\left(H_{k}\right)$. Now conditions 
(D) and (Fiv) guarantee that

$$
\gamma_{\infty}(t)=\lim _{l \rightarrow \infty} \gamma_{l}(t) \in \bar{B}\left(\gamma_{k}(t), \psi_{k}\right) \subseteq O_{k} \subseteq O_{n}
$$

Hence $\gamma_{\infty}(t) \in O_{n} \cap \mathscr{F}$.

Finally, we prove (v), (vi), and (vii) simultaneously. By (G), the set $F_{\infty}$ contains no dyadic numbers. Therefore, it suffices to verify the 'every portion of positive measure' condition of ( $v$ ) on all intervals $I \in \mathcal{P}_{k}$ for all $k \geq 2$. Further, to prove (vi), we may assume that $k \geq 2$, since $H_{1,1}=[0,1]$ is the only component of $H_{1}$ and contains all other $H_{k, j}$. Let $k \geq 2$ and $I \in \mathcal{P}_{k}$ be such that $I \cap F_{\infty} \neq \emptyset$. We claim that

$$
I \cap \gamma_{k-1}^{-1}(\mathscr{F}) \cap \bigcap_{i=1}^{k-1} W_{i}^{\mathrm{comp}} \neq \emptyset .
$$

Otherwise, applying (C) inductively for $k^{\prime} \geq k$ yields that $\left.\gamma_{\infty}\right|_{I}=\left.\gamma_{k^{\prime}}\right|_{I}=\left.\gamma_{k-1}\right|_{I}$ for all $k^{\prime} \geq k$. But this implies

$$
I \cap F_{\infty}=I \cap \gamma_{\infty}^{-1}(\mathscr{F}) \cap \bigcap_{i=1}^{\infty} W_{i}^{\mathrm{comp}} \subseteq I \cap \gamma_{k-1}^{-1}(\mathscr{F}) \cap \bigcap_{i=1}^{k-1} W_{i}^{\mathrm{comp}}=\emptyset,
$$

contrary to our assumption. This proves (4.26). By (Fi), there exists $j_{0} \in\left\{1, \ldots, p_{k}\right\}$ with $H_{k, j_{0}} \subseteq I$. For the proof of (vi), we write the next part of the argument for an arbitrary, fixed $j \in\left\{1, \ldots, p_{k}\right\}$. By (Fii), we have

$$
H_{k, j} \subseteq \bigcap_{i=1}^{k-1} W_{i}^{\mathrm{comp}} \text { and } \mathcal{L}\left(H_{k, j} \cap \gamma_{k}^{-1}\left(\mathscr{F} \cap O_{k}\right)\right) \geq \alpha_{k} .
$$

Applying (4.24), we infer

$$
\mathcal{L}\left(H_{k, j} \cap \gamma_{\infty}^{-1}\left(\mathscr{F} \cap O_{k}\right)\right) \geq \frac{\alpha_{k}}{2} .
$$

Finally, we apply (Gii) and (Eii) to derive

$$
\mathcal{L}\left(H_{k, j} \cap \gamma_{\infty}^{-1}\left(\mathscr{F} \cap O_{k}\right) \cap \bigcap_{i=1}^{\infty} W_{i}^{\mathrm{comp}}\right) \geq \frac{\alpha_{k}}{2}-\sum_{i=k}^{\infty} \mathcal{L}\left(W_{i}\right) \geq \frac{\alpha_{k}}{8}>0,
$$

which implies

$$
\mathcal{L}\left(F_{\infty} \cap \operatorname{Int}\left(H_{k, j}\right)\right)>0 .
$$

This proves (vi). Since $k \geq 2$ and $I \in \mathcal{P}_{k}$ were arbitrary and $H_{k, j_{0}} \subseteq I$, taking $j=j_{0}$ in (4.27) verifies (v) and further proves that the sets $\bigcup_{i=k}^{\infty} \operatorname{Int}\left(H_{i}\right) \cap F_{\infty}$ are dense in $F_{\infty}$ for all $k \in \mathbb{N}$. Hence, (vii) is also verified.

For each $t \in H$, let $\left(k_{n}(t)\right)_{n=1}^{\infty}$ be the increasing sequence of positive integers such that $t \in \operatorname{Int}\left(H_{k}\right)$ if and only if $k \in\left\{k_{n}(t): n \geq 1\right\}$. In other words, setting $k_{0}(t)=0$, we let

$$
k_{n}(t):=\min \left\{k>k_{n-1}(t): t \in \operatorname{Int}\left(H_{k}\right)\right\}, \quad t \in H, n \geq 1 .
$$

In places where the relevant point $t \in H$ is clear, we often shorten $k_{n}(t)$ to $k_{n}$.

Remark 4.5. Recall from (Fiii) that any two components $H_{k, j}, H_{l, i}$ of $H_{k}$ and $H_{l}$, respectively, with $k \neq l$ are either pairwise disjoint or strongly nested in the sense that one is contained in the interior of the other. This implies the following additional property, which we will use later: if $t \in H, k_{n}:=k_{n}(t)$ for $n \geq 1$, and $s \in \operatorname{Int}\left(H_{k_{m}, j_{m}}\right)$ for some $m \geq 1$, then

$$
k_{n}(s)=k_{n}(t)=k_{n} \text { for all } 1 \leq n \leq m .
$$


Let $t \in H$ and $k_{n}=k_{n}(t)$. By (Fvi), we have that $\beta_{k_{n}}(t)>\beta_{k_{n-1}}(t)$ for each $n \geq 2$. This implies that for each fixed $m \geq 1$, the sequence $\left.\beta_{k_{n}}(t)\right|_{m}$ eventually becomes constant. Define the infinite sequence $\beta(t)=\left(i_{m}\right)_{m=1}^{\infty}$ by the condition

$$
\left.\beta(t)\right|_{m}=\left.\lim _{n \rightarrow \infty} \beta_{k_{n}}(t)\right|_{m} \quad \text { for each } m \geq 1, \text { where } t \in H .
$$

Note for future reference that

$$
\left.\beta_{k_{n}}(t)\right|_{m} \leq\left.\beta(t)\right|_{m} \quad \text { for all } n, m \geq 1, \text { where } t \in H .
$$

Recall from Lemma 4.4, part (ii), that for each $t \in H$ and $k \in \mathbb{N}$, the derivative $\gamma_{k}^{\prime}(t)$ exists. The next lemma gives an estimate of how close the derivatives of $\gamma_{k}$ on $H$ are in terms of the function $\zeta$ defined in (4.2).

Lemma 4.6. Let $t \in H$ (see (4.25)) and $k_{n}:=k_{n}(t)$ be defined according to (4.28). Let $k_{1} \leq k \leq l$, and let $p, q \geq 1$ be maximal such that $k_{p} \leq k$ and $k_{q} \leq l$. Then

$$
\left\|\gamma_{l}^{\prime}(t)-\gamma_{k}^{\prime}(t)\right\| \leq 2 \zeta\left(\beta_{k_{q}}(t), \beta_{k_{p}}(t)\right)+7 \cdot 2^{-k_{p}} .
$$

Proof. Clearly, $1 \leq k_{p} \leq k_{q}$. By (Hi), we have

$$
\left\|\gamma_{l}^{\prime}(t)-\gamma_{k_{q}}^{\prime}(t)\right\| \leq \sum_{m=k_{q}+1}^{l} 2^{-m} \leq 2^{-k_{q}} \leq 2^{-k_{p}},
$$

and similarly

$$
\left\|\gamma_{k}^{\prime}(t)-\gamma_{k_{p}}^{\prime}(t)\right\| \leq 2^{-k_{p}}
$$

To obtain an estimate for $\left\|\gamma_{k_{q}}^{\prime}(t)-\gamma_{k_{p}}^{\prime}(t)\right\|$, we compare separately the magnitudes and directions of these vectors. By (Hi) and (Hii), the magnitudes differ by

$$
\left|\left\|\gamma_{k_{q}}^{\prime}(t)\right\|-\left\|\gamma_{k_{p}}^{\prime}(t)\right\|\right| \leq \sum_{m=k_{p}+1}^{k_{q}} 2^{-m} \leq 2^{-k_{p}},
$$

and with (Hiv), we can bound the difference of directions by

$$
\left\|\theta\left(\gamma_{k_{q}}^{\prime}(t)\right)-\theta\left(\gamma_{k_{p}}^{\prime}(t)\right)\right\| \leq \zeta\left(\beta_{k_{q}}(t), \beta_{k_{p}}(t)\right)+2 \cdot 2^{-k_{p}} .
$$

Combining the last two inequalities and using that $\left\|\gamma_{n}^{\prime}(t)\right\| \leq 2$, from (A), for all $n \geq 1$, we deduce

$$
\left\|\gamma_{k_{q}}^{\prime}(t)-\gamma_{k_{p}}^{\prime}(t)\right\| \leq 2^{-k_{p}}+2\left(\zeta\left(\beta_{k_{q}}, \beta_{k_{p}}(t)\right)+2 \cdot 2^{-k_{p}}\right) .
$$

The inequality of Lemma 4.6 now follows by the triangle inequality.

The previous lemma enables us to establish the convergence of the derivatives $\gamma_{k}^{\prime}(t)$ at points $t \in H$.

Lemma 4.7. Let $t \in H$. Then the sequence $\left(\gamma_{k}^{\prime}(t)\right)_{k=1}^{\infty}$ converges and

$$
\theta\left(\lim _{k \rightarrow \infty} \gamma_{k}^{\prime}(t)\right)=\bigcap_{n \geq 1} S_{\beta(t) \mid n},
$$

where $\beta(t)=\lim _{n \rightarrow \infty} \beta_{k_{n}}(t)$ and $k_{n}=k_{n}(t)$ are defined in (4.30) and (4.28), respectively. 
Proof. Given $\varepsilon>0$, choose $M \in \mathbb{N}$ such that $2^{-M+2} \sqrt{d}<\varepsilon$, i.e. the diameter $\zeta(\beta, \beta)$ of any $S_{\beta}$ with $\beta \in T_{n}, n \geq M$, is less than $\varepsilon$ (see Definition 4.1). Let $N>M$ be such that for any $n \geq N$, it holds that $\left.\beta_{k_{n}}(t)\right|_{M}=\left.\beta(t)\right|_{M}$.

Given $l>k \geq k_{N}$, we choose $p, q \in \mathbb{N}$ maximal so that $k_{p} \leq k$ and $k_{q} \leq l$. Then, by Lemma 4.6, we have

$$
\left\|\gamma_{l}^{\prime}(t)-\gamma_{k}^{\prime}(t)\right\| \leq 2 \zeta\left(\beta_{k_{q}}(t), \beta_{k_{p}}(t)\right)+7 \cdot 2^{-k_{p}}<2 \varepsilon+7 \varepsilon .
$$

Here, we used that $p, q \geq N$ to deduce $\left.\beta_{k_{p}}(t)\right|_{M}=\left.\beta_{k_{q}}(t)\right|_{M}=\left.\beta(t)\right|_{M}$, and subsequently

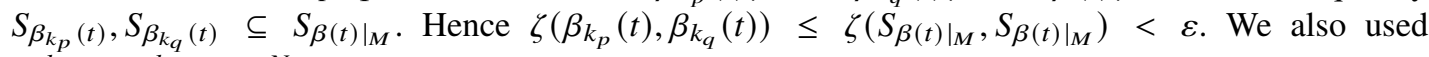
$2^{-k_{p}} \leq 2^{-k_{N}} \leq 2^{-N}<\varepsilon$.

We thus conclude that $\left(\gamma_{k}^{\prime}(t)\right)_{k=1}^{\infty}$ is a Cauchy sequence and hence converges. Moreover, for any $p \geq N$, we have, by (Hiv), that $\theta\left(\gamma_{k_{p}}^{\prime}(t)\right) \in B\left(S_{\beta_{k_{p}}(t)}, 2^{-k_{p}}\right) \subseteq B\left(S_{\left.\beta(t)\right|_{M}}, 2^{-k_{p}}\right)$. Letting $p \rightarrow \infty$, we conclude that the vector $x:=\theta\left(\lim _{k \rightarrow \infty} \gamma_{k}^{\prime}(t)\right)$ belongs to $S_{\left.\beta(t)\right|_{M}}$. Since $M \in \mathbb{N}$ could have been chosen arbitrarily large, this proves $x \in \bigcap_{n=1}^{\infty} S_{\left.\beta(t)\right|_{n}}$. It is clear that the latter has diameter 0 , and thus the statement of the lemma follows.

For each $k \geq 1$, let us recall (4.25) and define

$$
\Omega_{k}:=\left\{t \in H: \exists \sigma=\sigma(t, k)>0 \text { s.t. }\left.\beta(s)\right|_{k} \leq\left.\beta(t)\right|_{k} \text { for all } s \in[t-\sigma, t+\sigma] \cap H\right\}
$$

and

$$
E_{\infty}:=\bigcap_{k=1}^{\infty} \Omega_{k} .
$$

We now show that each of the sets $\Omega_{k}$ is non-empty and, moreover, that each $\Omega_{k} \cap F_{\infty}$ contains a relatively open and dense subset of $H \cap F_{\infty}$. Together with Lemma 4.4(vii), this will imply that $E_{\infty} \cap F_{\infty}$ is relatively residual in $F_{\infty}$.

Lemma 4.8. The sets $\Omega_{k}, E_{\infty}$ and $F_{\infty}$ defined in (4.33), (4.34), and (4.25) have the following properties:

(i) For each $k \geq 1$, the set $\Omega_{k} \cap F_{\infty}$ contains a relatively open and dense subset of $H \cap F_{\infty}$.

(ii) The set $E_{\infty} \cap F_{\infty}$ is a non-empty, relatively residual subset of $F_{\infty}$.

Proof. A subset $R$ of a topological space $X$ contains an open, dense set if and only if $R$ intersects every non-empty, open set in a set of non-empty interior. We prove part (i) by verifying this equivalent condition for the sets $R=\Omega_{k} \cap F_{\infty}$ and topological space $X=H \cap F_{\infty}$ with the subspace topology inherited from $[0,1]$. Thus, fixing $k \geq 1$ and an open interval $U \subseteq \mathbb{R}$ with $U \cap H \cap F_{\infty} \neq \emptyset$, our task is to find an open interval $V \subseteq U$ such that

$$
\emptyset \neq V \cap H \cap F_{\infty} \subseteq \Omega_{k} \cap F_{\infty}
$$

Since $U \cap H \neq \emptyset$, the set

$$
\left\{\left.\beta(r)\right|_{k}: r \in U \cap H\right\}
$$

is a finite, non-empty set. Therefore, there exists $t \in U \cap H$ such that

$$
\left.\beta(t)\right|_{k}=\max \left\{\left.\beta(r)\right|_{k}: r \in U \cap H\right\} .
$$

Note that a priori, we do not know whether $t$ belongs to $F_{\infty}$. Let $k_{n}=k_{n}(t)$ be defined by (4.28). We then have $\beta(t)=\lim _{n \rightarrow \infty} \beta_{k_{n}}(t)$; see (4.30). Therefore, we may choose $n_{0} \in \mathbb{N}$ large enough that $\left.\beta_{k_{n}}(t)\right|_{k}=\left.\beta(t)\right|_{k}$ is constant for all $n \geq n_{0}$. Fix $n \geq n_{0}$, and consider the component $H_{k_{n}, j_{n}}$ of $H_{k_{n}}$ containing $t$. We additionally take $n$ sufficiently large that $H_{k_{n}, j_{n}} \subseteq U$. Now we seek to verify (4.35) for $V:=\operatorname{Int}\left(H_{k_{n}, j_{n}}\right) \subseteq U$. First note that the set $V \cap H \cap F_{\infty}$ is non-empty. Indeed, by Lemma 4.4, part (vi), 
the set $V \cap F_{\infty}$ is a non-empty, relatively open subset of $F_{\infty}$. Therefore, by Lemma 4.4, part (vii), it has a non-empty intersection with $H$. Let $s \in V \cap H \cap F_{\infty}$. Then, by Remark 4.5, we have $k_{i}(s)=k_{i}(t)=k_{i}$ for $1 \leq i \leq n$. Hence, using (4.31) and the choice (4.36) of $t$, we get

$$
\left.\beta(s)\right|_{k} \geq\left.\beta_{k_{n}}(s)\right|_{k}=\left.\beta_{k_{n}}(t)\right|_{k}=\left.\beta(t)\right|_{k} \geq\left.\beta(s)\right|_{k} .
$$

We conclude that $\left.\beta(s)\right|_{k}=\left.\beta(t)\right|_{k}$. Taking $\sigma=\sigma(s, k)>0$ sufficiently small that $[s-\sigma, s+\sigma] \subseteq U$, and using (4.36), we verify that $s \in \Omega_{k}$. Hence $s \in \Omega_{k} \cap F_{\infty}$.

We turn our attention now to part (ii). From part (i), it follows that $E_{\infty} \cap F_{\infty}(:=Z)$ is a relatively residual subset of $H \cap F_{\infty}(:=Y)$. Recall, in addition, that $H \cap F_{\infty}$ is a relatively residual subset of $F_{\infty}(:=X)$ and that $F_{\infty}$ is closed (Lemma 4.4, parts (vii) and (iii)) and thus a Baire space in its own right. Therefore, to prove (ii), it suffices to recall the following general topological statement, which may be verified easily using [13, §10 IV Theorem 1].

Let $X$ be a topological space, $Y \subseteq X$ be a residual subset of $X$, and $Z \subseteq Y$ be a relatively residual subset of $Y$. Then $Z$ is a residual subset of $X$.

We are now ready to take an important step and verify that the limit curve $\gamma_{\infty}=\lim \gamma_{k}$ is differentiable everywhere in $E_{\infty}$ (4.34) and that its derivative is the limit of derivatives of $\gamma_{k}$.

Lemma 4.9. Let $t \in E_{\infty}$. Then the Lipschitz curve $\gamma_{\infty}$ is differentiable at $t$ with

$$
\gamma_{\infty}^{\prime}(t)=\lim _{k \rightarrow \infty} \gamma_{k}^{\prime}(t)
$$

Moreover, we have

$$
\lim _{\delta \rightarrow 0} \operatorname{osc}_{\gamma_{\infty}^{\prime}}([t-\delta, t+\delta])=0 .
$$

Proof. Fix $\varepsilon>0$. Let $N \in \mathbb{N}$ be sufficiently large that $2^{-N+2} \sqrt{d}<\varepsilon$ : i.e. the diameter of any $S_{\beta}$ with $\beta \in T_{n}, n \geq N$, is less than $\varepsilon$. As $t \in E_{\infty} \subseteq \Omega_{N}$, let $\sigma(t, N)>0$ be given by the definition (4.33) of $\Omega_{N}$.

Recall $\Omega_{N} \subseteq H$, so $t \in H$. Let $\kappa_{n}=k_{n}(t)$ be the sequence of indices defined by (4.28). For each $n \in \mathbb{N}$, let $j_{n} \in\left\{1, \ldots, p_{n}\right\}$ be the index with $t \in \operatorname{Int}\left(H_{\kappa_{n}, j_{n}}\right)$. By Lemma 4.7, there exists $L(t)=\lim _{n \rightarrow \infty} \gamma_{\kappa_{n}}^{\prime}(t)$. Choose $M \geq N$ sufficiently large that

○ $\left.\beta_{\kappa_{m}}(t)\right|_{N}=\left.\beta(t)\right|_{N}$ is constant for all $m \geq M$,

○ $H_{K_{M}, j_{M}} \subseteq[t-\sigma(t, N), t+\sigma(t, N)]$,

$\circ\left\|\gamma_{K_{M}}^{\prime}(t)-L(t)\right\| \leq \varepsilon$.

Choose $\eta$ sufficiently small that $[t-\eta, t+\eta] \subseteq H_{K_{M}, j_{M}}$. Then, by (Fv), we have $\beta_{K_{M}}(s)=\beta_{K_{M}}(t)=: \beta$ for all $s \in[t-\eta, t+\eta]$. By (4.29) of Remark 4.5, we conclude that $k_{n}(s)=k_{n}(t)=\kappa_{n}$ for all $s \in[t-\eta, t+\eta]$ and $1 \leq n \leq M$.

Let $s \in[t-\eta, t+\eta]$ and $l \geq \kappa_{M}$, and choose $q \in \mathbb{N}$ maximal so that $k_{q}(s) \leq l$. As $l \geq \kappa_{M}=k_{M}(s)$, we conclude that $q \geq M$. Using, in addition, (Fvi) with $s \in H_{K_{M}} \cap H_{k_{q}}, t \in \Omega_{N}$, and (4.31), we get

$$
\left.\beta_{k_{q}}(s)\right|_{N} \geq\left.\beta_{\kappa_{M}}(s)\right|_{N}=\left.\beta_{\kappa_{M}}(t)\right|_{N}=\left.\beta(t)\right|_{N} \geq\left.\beta(s)\right|_{N} \geq\left.\beta_{k_{q}}(s)\right|_{N} .
$$

Therefore, $\left.\beta_{k_{q}}(s)\right|_{N}=\left.\beta_{\kappa_{M}}(s)\right|_{N}=\left.\beta(s)\right|_{N}$ so that

$$
\zeta\left(\beta_{k_{q}}(s), \beta_{\kappa_{M}}(s)\right) \leq \zeta\left(\left.\beta(s)\right|_{N},\left.\beta(s)\right|_{N}\right)<\varepsilon .
$$

Then, applying Lemma 4.6, we get

$$
\left\|\gamma_{l}^{\prime}(s)-\gamma_{K_{M}}^{\prime}(s)\right\| \leq 2 \zeta\left(\beta_{k_{q}}(s), \beta_{\kappa_{M}}(s)\right)+7 \cdot 2^{-\kappa_{M}}<9 \varepsilon .
$$


From this, we conclude that $\operatorname{Lip}\left(\left.\left(\gamma_{l}-\gamma_{\kappa_{M}}\right)\right|_{[t-\eta, t+\eta]}\right) \leq 9 \varepsilon$. Since $\gamma_{l}$ converges uniformly to $\gamma_{\infty}$, we deduce that $\operatorname{Lip}\left(\left.\left(\gamma_{\infty}-\gamma_{\kappa_{M}}\right)\right|_{[t-\eta, t+\eta]}\right) \leq 9 \varepsilon$. Further, by (Hiv), (Hiii), and (A), we have

$$
\operatorname{osc}_{\gamma_{\kappa_{M}}^{\prime}}([t-\eta, t+\eta]) \leq 2 \operatorname{diam}\left(B\left(S_{\beta_{\kappa_{M}}}, 2^{-\kappa_{M}}\right)\right)=2\left(2 \cdot 2^{-\kappa_{M}}+\zeta\left(\beta_{\kappa_{M}}, \beta_{\kappa_{M}}\right)\right) \leq 4 \varepsilon .
$$

It follows that for all $h \in[-\eta, \eta]$,

$$
\left\|\gamma_{\kappa_{M}}(t+h)-\gamma_{\kappa_{M}}(t)-h \gamma_{K_{M}}^{\prime}(t)\right\| \leq 4 \varepsilon|h| .
$$

Using

$$
\left\|\frac{\gamma_{\infty}(t+h)-\gamma_{\infty}(t)}{h}-\gamma_{K_{M}}^{\prime}(t)\right\| \leq\left\|\frac{\left(\gamma_{\infty}-\gamma_{\kappa_{M}}\right)(t+h)-\left(\gamma_{\infty}-\gamma_{\kappa_{M}}\right)(t)}{h}\right\|+\left\|\frac{\gamma_{\kappa_{M}}(t+h)-\gamma_{\kappa_{M}}(t)}{h}-\gamma_{K_{M}}^{\prime}(t)\right\|,
$$

we now derive, for all $h \in[-\eta, \eta] \backslash\{0\}$,

$$
\left\|\frac{\gamma_{\infty}(t+h)-\gamma_{\infty}(t)}{h}-L(t)\right\| \leq 9 \varepsilon+4 \varepsilon+\left\|\gamma_{\kappa_{M}}^{\prime}(t)-L(t)\right\| \leq 14 \varepsilon
$$

Since $\varepsilon>0$ was arbitrary, this verifies the differentiability of $\gamma_{\infty}$ at $t$ with $\gamma_{\infty}^{\prime}(t)=L(t)$. For the 'moreover' part of the lemma, we observe that

$$
\operatorname{osc}_{\gamma_{\infty}^{\prime}}([t-\eta, t+\eta]) \leq 2 \operatorname{Lip}\left(\left.\left(\gamma_{\infty}-\gamma_{\kappa_{M}}\right)\right|_{[t-\eta, t+\eta]}\right)+\operatorname{osc}_{\gamma_{\kappa_{M}}^{\prime}}([t-\eta, t+\eta]) \leq 18 \varepsilon+4 \varepsilon .
$$

We now reparameterise the curve $\gamma_{\infty}$ to obtain a curve $\gamma: I(\gamma) \rightarrow(0,1)^{d}$ satisfying the conclusions of Theorem 2.11. Let

$$
\ell\left(\gamma_{\infty}\right):=\int_{0}^{1}\left\|\gamma_{\infty}^{\prime}(s)\right\| d s
$$

denote the length of the curve $\gamma_{\infty}$. Define a mapping $\varphi:[0,1] \rightarrow\left[0, \ell\left(\gamma_{\infty}\right)\right]$ by

$$
\varphi(t)=\int_{0}^{t}\left\|\gamma_{\infty}^{\prime}(s)\right\| d s, \quad t \in[0,1] .
$$

Lemma 4.10. The function $\varphi:[0,1] \rightarrow\left[0, \ell\left(\gamma_{\infty}\right)\right]$ has the following properties:

(i) The function $\varphi$ is bilipschitz with $\operatorname{Lip}(\varphi), \operatorname{Lip}\left(\varphi^{-1}\right) \leq 2$.

(ii) There is a set $X \subseteq[0,1]$ of full measure with $E_{\infty} \subseteq X$ such that for every $t \in X$, both $\gamma_{\infty}$ and $\varphi$ are differentiable at $t$ and

$$
\varphi^{\prime}(t)=\left\|\gamma_{\infty}^{\prime}(t)\right\| \geq \frac{1}{2}
$$

Proof. Part (i) follows from Lemma 4.3(iii) and (A). For part (ii), let $X$ be defined as the set of points $s \in$ $[0,1] \backslash \bigcup_{i=1}^{\infty} M_{i}$ at which all curves $\gamma_{k}$ with $k \in \mathbb{N} \cup\{\infty\}$ are differentiable and $\gamma_{\infty}^{\prime}(s)=\lim _{k \rightarrow \infty} \gamma_{k}^{\prime}(s)$. The inequality $\geq \frac{1}{2}$ in the statement is now a consequence of (A). Recalling that the sets $M_{i}$ are finite, it follows immediately from Lemma 4.3(iii) that $X$ has full measure. Further, from Lemma 4.9 and $E_{\infty} \subseteq H \subseteq[0,1] \backslash \bigcup_{i=1}^{\infty} M_{i}$, we derive that $X$ contains $E_{\infty}$. Fix $t \in X$ and $\varepsilon \in(0,1 / 4)$, and let $k \in \mathbb{N}$ be large enough that $2^{-k} \leq \varepsilon$ and $\left\|\gamma_{k}^{\prime}(t)-\gamma_{\infty}^{\prime}(t)\right\| \leq \varepsilon$. Next, choose $\delta>0$ small enough that $[t-\delta, t+\delta]$ is contained in a single component of $[0,1] \backslash M_{k}$. From (Hi)-(Hiii), it follows that

$$
\left|\left\|\gamma_{l}^{\prime}(s)\right\|-\left\|\gamma_{k}^{\prime}(t)\right\|\right| \leq 2^{-k} \leq \varepsilon
$$


for all $l \geq k$ and all $s \in[t-\delta, t+\delta] \cap X$, implying

$$
\left|\left\|\gamma_{\infty}^{\prime}(s)\right\|-\left\|\gamma_{k}^{\prime}(t)\right\|\right| \leq \varepsilon
$$

for all such $s$. Hence, for almost all $s \in[t-\delta, t+\delta]$, we have

$$
\left|\left\|\gamma_{\infty}^{\prime}(s)\right\|-\left\|\gamma_{\infty}^{\prime}(t)\right\|\right| \leq\left|\left\|\gamma_{\infty}^{\prime}(s)\right\|-\left\|\gamma_{k}^{\prime}(t)\right\|\right|+\varepsilon \leq 2 \varepsilon,
$$

and therefore, for all $h \in[-\delta, \delta]$, we have

$$
\left|\varphi(t+h)-\varphi(t)-h \cdot\left\|\gamma_{\infty}^{\prime}(t)\right\|\right| \leq \int_{t}^{t+h}\left|\left\|\gamma_{\infty}^{\prime}(s)\right\|-\left\|\gamma_{\infty}^{\prime}(t)\right\|\right| d s \leq 2 \varepsilon|h| .
$$

We now use results and constructions of Section 4 to finish the proof of Theorem 2.11.

Proof of Theorem 2.11. We find a curve $\gamma$ satisfying all assertions of Theorem 2.11 except that its domain is an interval $I(\gamma)$ and not necessarily $[0,1]$. It is then a trivial matter to adjust $\gamma$ so that its domain is $[0,1]$ and all assertions of the theorem remain valid. We comment briefly on the required modification at the very end.

From Lemma 4.10(ii) and an appropriate form of the inverse function theorem, it follows that

$$
\left(\varphi^{-1}\right)^{\prime}(\varphi(r))=\frac{1}{\left\|\gamma_{\infty}^{\prime}(r)\right\|}
$$

for all $r \in X$, where $X$ and $\varphi$ are given by Lemma 4.10. More precisely, (4.37) is obtained by an application of [23, Theorem 1.2] to $U=(0,1), n=1, x_{0}=r \in X$, and $f=\varphi$. Note that the condition $f^{\prime}\left(x_{0}\right)=\varphi^{\prime}(r) \in \operatorname{Isom}(\mathbb{R}, \mathbb{R})$ is satisfied due to Lemma 4.10(ii). Since in this case, $f=\varphi$ is invertible, the function $h$ given by the conclusion of [23, Theorem 1.2] necessarily coincides with $\varphi^{-1}$ on its domain.

We recall sets $E_{\infty}$ and $F_{\infty}$ from (4.34) and (4.25) to define

$$
F:=\varphi\left(F_{\infty}\right), \quad E:=\varphi\left(E_{\infty} \cap F_{\infty}\right)
$$

and $\gamma:\left[0, \ell\left(\gamma_{\infty}\right)\right] \rightarrow(0,1)^{d}$ by

$$
\gamma(t)=\gamma_{\infty}\left(\varphi^{-1}(t)\right)
$$

By Lemmata 4.4 (v) and 4.8 (ii), the sets $E$ and $F$ are non-empty. We verify the assertions (i)-(v) of Theorem 2.11 for $F, E$, and $\gamma$. The properties (i) and (ii) are invariant under bilipschitz transformations. Therefore, $F$ and $E$ inherit these properties from $F_{\infty}$ and $E_{\infty} \cap F_{\infty}$; see Lemmata 4.4 (v) and 4.8 (ii). Moreover, (v) is immediate from the definitions of $\gamma, E, F$, and Lemma 4.4(iv). To complete the proof, we verify (iii) and (iv). Fix $t \in \varphi(X)$. Then $t=\varphi(r)$ for some $r \in X$. Applying (4.37), we conclude that $\varphi^{-1}$ is differentiable at $t$ with derivative $\left(\varphi^{-1}\right)^{\prime}(t)=\frac{1}{\left\|\gamma_{\infty}^{\prime}(r)\right\|}$. Moreover, $\gamma_{\infty}$ is differentiable at $\varphi^{-1}(t)$ by Lemma 4.10. It follows that $\gamma$ is differentiable at $t$ with

$$
\gamma^{\prime}(t)=\gamma_{\infty}^{\prime}\left(\varphi^{-1}(t)\right) \cdot\left(\varphi^{-1}\right)^{\prime}(t)=\gamma_{\infty}^{\prime}(r) \cdot \frac{1}{\left\|\gamma_{\infty}^{\prime}(r)\right\|} .
$$

Clearly, from the above, we also have $\left\|\gamma^{\prime}(t)\right\|=1$. Since $E \subseteq \varphi(X)$, part (iii) is satisfied. For $t_{0}=\varphi\left(r_{0}\right) \in E$ and any $t, s \in\left[t_{0}-\delta, t_{0}+\delta\right] \cap \varphi(X)$, Lemma 4.10(i) implies that the preimages $r_{t}:=\varphi^{-1}(t)$ and $r_{s}:=\varphi^{-1}(s)$ belong to $\varphi^{-1}\left[t_{0}-\delta, t_{0}+\delta\right] \cap X \subseteq\left[r_{0}-2 \delta, r_{0}+2 \delta\right]$, and then (4.37), 
together with Lemma 4.3(ii), implies $\left|\left(\varphi^{-1}\right)^{\prime}(t)-\left(\varphi^{-1}\right)^{\prime}(s)\right| \leq 4 \operatorname{osc}_{\gamma_{\infty}^{\prime}}\left(\left[r_{0}-2 \delta, r_{0}+2 \delta\right]\right)$. Therefore, we obtain

$$
\begin{aligned}
\left\|\gamma^{\prime}(t)-\gamma^{\prime}(s)\right\| & =\left\|\gamma_{\infty}^{\prime}\left(r_{t}\right) \cdot\left(\varphi^{-1}\right)^{\prime}(t)-\gamma_{\infty}^{\prime}\left(r_{s}\right) \cdot\left(\varphi^{-1}\right)^{\prime}(s)\right\| \\
& \leq\left\|\gamma_{\infty}^{\prime}\left(r_{t}\right)\right\|\left|\left(\varphi^{-1}\right)^{\prime}(t)-\left(\varphi^{-1}\right)^{\prime}(s)\right|+\left|\left(\varphi^{-1}\right)^{\prime}(s)\right| \operatorname{osc}_{\gamma_{\infty}^{\prime}}\left(\left[r_{0}-2 \delta, r_{0}+2 \delta\right]\right) \\
& \leq 10 \operatorname{osc}_{\gamma_{\infty}^{\prime}}\left(\left[r_{0}-2 \delta, r_{0}+2 \delta\right]\right),
\end{aligned}
$$

where, for the last inequality, we used that both $\operatorname{Lip}\left(\varphi^{-1}\right)$ and $\operatorname{Lip}\left(\gamma_{\infty}\right)$ are bounded from above by 2; see Lemmata 4.3(i) and 4.10(i).

The proof of part (iv) is now completed by the 'moreover' conclusion of Lemma 4.9.

Let us now comment on why we may assume that the domain $I(\gamma)$ of $\gamma$ is the interval $[0,1]$, as in the statement of Theorem 2.11. Note that $I(\gamma)$ has the form $[0, a]$ for some $a:=\ell\left(\gamma_{\infty}\right)>0$. If $a \geq 1$, then we choose a closed interval $J \subseteq(0, a)$ of length strictly less than one such that the endpoints of $J$ are density points of $F$. We then redefine the sets $F$ and $E$ by intersecting with $J$. Finally, we choose a closed interval $J^{\prime} \subseteq[0, a]$ of length one with $J \subseteq \operatorname{Int}\left(J^{\prime}\right)$ and redefine $\gamma$ by restricting to $J^{\prime}$ and then shifting so that $\gamma$ is defined on $[0,1]$. If $a<1$, then we extend the curve $\gamma$ arbitrarily to [0,1] and leave the sets $F \subseteq[0, a]$ and $E \subseteq[0, a]$ unchanged. In both cases, all assertions (i)-(v) of Theorem 2.11 are preserved.

\section{Typical non-differentiability on coverable sets}

In this section, we prove Theorem 2.7 ; that is, we show that any set in $(0,1)^{d}$ that may be covered by a countable union of closed, purely unrectifiable sets avoids, for the typical function $f \in \operatorname{Lip}_{1}\left([0,1]^{d}\right)$, the set of points where $f$ has a directional derivative.

Notation. We will write $\operatorname{Lip}\left([0,1]^{d}\right)$ for the set of all Lipschitz functions $[0,1]^{d} \rightarrow \mathbb{R}$. Further, recall that for a subset $U \subseteq[0,1]^{d}$, we let $C^{1}(U)$ denote the set of continuous functions $f:[0,1]^{d} \rightarrow \mathbb{R}$ with the property that $\left.f\right|_{\operatorname{Int}(U)}$ is $C^{1}$.

The following lemma is a simplification of [15, Lemma 2.3], in the case when $P \subseteq[0,1]^{d}$ is a closed set. We also only state it in the case when the function $\omega_{0}(t)$ of [15, Lemma 2.3] is constant.

Lemma 5.1. Suppose that $P \subseteq H \subseteq(0,1)^{d}$, where $P$ is closed and $H$ is open; the function $g:(0,1)^{d} \rightarrow$ $\mathbb{R}$ belongs to $C^{1}(H) ;$ and $\omega_{0}, \eta \in(0,1)$. Then there are $\xi_{0}, r_{0} \in\left(0, \omega_{0} / 2\right]$ such that if $h:[0,1]^{d} \rightarrow \mathbb{R}$ satisfies

$$
|h(x)-g(x)| \leq 2 \xi_{0} \text { for all } x \in[0,1]^{d},
$$

then for all $x \in P$ and $\|y\| \leq r_{0}$, it holds that

$$
|h(x+y)-h(x)-\langle\nabla g(x), y\rangle| \leq \eta r_{0} .
$$

Proof. Denote $\rho_{H}(x):=\operatorname{dist}\left(x,[0,1]^{d} \backslash H\right)$. Let $\Psi$ be the set of functions $\psi \in \operatorname{Lip}_{1}\left([0,1]^{d}\right)$ satisfying $0 \leq \psi(x) \leq \frac{1}{2} \min \left(\rho_{H}(x), \omega_{0}\right)$ and such that

$$
\|\nabla g(y)-\nabla g(z)\| \leq \frac{1}{2} \eta \text { whenever } x \in H \text { and } \max (\|y-x\|,\|z-x\|)<\psi(x) .
$$

Since $0 \in \Psi$, the function $\varphi(x):=\sup \{\psi(x): \psi \in \Psi\}$ is well-defined. We also have $\varphi \in \Psi$, since for any $x, y, z$ satisfying $x \in H$ and $\max (\|y-x\|,\|z-x\|)<\varphi(x)$, there is $\psi \in \Psi$ such that $\max (\|y-x\|,\|z-x\|)<\psi(x)$ and hence $\|\nabla g(y)-\nabla g(z)\| \leq \frac{1}{2} \eta$.

Let $w \in H$ be arbitrary. Choose $\varepsilon_{w} \in\left(0, \omega_{0} / 2\right)$ such that $B\left(w, 3 \varepsilon_{w}\right) \subseteq H$ and the bound $\|\nabla g(y)-\nabla g(z)\| \leq \frac{1}{2} \eta$ holds for $y, z \in B\left(w, 2 \varepsilon_{w}\right)$. Then the function defined by $\psi_{w}(x):=\max \left(0, \varepsilon_{w}-\right.$ $\|x-w\|)$ satisfies $\psi_{w}=0$ outside of the ball $B\left(w, \varepsilon_{w}\right)$, and $0 \leq \psi_{w}(x) \leq \varepsilon_{w} \leq \frac{1}{2} \min \left(\rho_{H}(x), \omega_{0}\right)$ for all $x \in B\left(w, \varepsilon_{w}\right)$. This, together with the choice of $\varepsilon_{w}$, clearly ensures that (5.3) is satisfied for $\psi=\psi_{w}$. 
Hence, $\psi_{w} \in \Psi$, and we infer that $\varphi(w) \geq \psi_{w}(w)=\varepsilon_{w}>0$. Consequently, $\varphi$ is strictly positive on $H$. Let $\varphi_{0}=\inf \{\varphi(x): x \in P\}$; as $P$ is compact, we have that $0<\varphi_{0} \leq \frac{1}{2} \omega_{0}$. Furthermore, whenever $x \in P$ and $\|y\|<\varphi_{0}$, it holds that

$$
|g(x+y)-g(x)-\langle\nabla g(x), y\rangle| \leq\|y\| \sup _{z \in B(x,\|y\|)}\|\nabla g(z)-\nabla g(x)\| \leq \frac{1}{2} \eta\|y\| .
$$

To prove (5.2), we let $r_{0}:=\varphi_{0} / 2 \in\left(0, \omega_{0} / 2\right]$ and $\xi_{0}:=\varphi_{0} \eta / 16=r_{0} \eta / 8 \in\left(0, \omega_{0} / 2\right]$ and consider an arbitrary function $h:[0,1]^{d} \rightarrow \mathbb{R}$ satisfying (5.1). Then, whenever $x \in P$ and $\|y\| \leq r_{0}<\varphi_{0} \leq \varphi(x)$, we have

$$
\begin{aligned}
|h(x+y)-h(x)-\langle\nabla g(x), y\rangle| & \leq 4 \xi_{0}+|g(x+y)-g(x)-\langle\nabla g(x), y\rangle| \\
& \leq 4 \xi_{0}+\frac{1}{2} \eta\|y\| \leq \eta r_{0} .
\end{aligned}
$$

Hence, [15, Lemma 2.9] may be restated in the following way, in the case of a compact, purely unrectifiable set $P$ (note that such sets are automatically uniformly purely unrectifiable; see $[15,1]$ ).

Lemma 5.2. Suppose $P \subseteq H \subseteq(0,1)^{d}$; $P$ is a closed, uniformly purely unrectifiable set; $H$ is open; $\omega_{0} \in(0,1) ;$ and $f \in \operatorname{Lip}\left([0,1]^{d}\right) \cap C^{1}(H)$. Then for every $e \in \mathbb{R}^{d}$ and $\eta>0$, there are $g:[0,1]^{d} \rightarrow \mathbb{R}$, $\xi_{0}, r \in\left(0, \omega_{0}\right)$, and an open set $U \subseteq(0,1)^{d}$ such that

(i) $P \subseteq U \subseteq H$,

(ii) $g \in \operatorname{Lip}\left([0,1]^{d}\right) \cap C^{1}(U) \cdot \operatorname{Lip}(g) \leq \max (\operatorname{Lip}(f)$, $\|e\|)+\eta$, and $\|g-f\|_{\infty} \leq \omega_{0}$.

(iii) If a function $h:[0,1]^{d} \rightarrow \mathbb{R}$ satisfies $|h(x)-g(x)| \leq 2 \xi_{0}$ for all $x \in[0,1]^{d}$, then $\sup _{\|y\| \leq r}|h(x+y)-h(x)-\langle e, y\rangle| \leq \eta r$ for all $x \in P$.

We are now ready to prove Theorem 2.7 , which we restate here, in a slightly different form, for the reader's convenience.

Theorem 5.3 (restatement of Theorem 2.7). Let $P \subseteq(0,1)^{d}$ be an $F_{\sigma}$, purely unrectifiable set. Then a typical $f \in \operatorname{Lip}_{1}\left([0,1]^{d}\right)$ has no directional derivatives at every point of $P$; and, moreover, for $a$ typical $f \in \operatorname{Lip}_{1}\left([0,1]^{d}\right)$, it holds that $\mathcal{D} f(x, v)=[-1,1]$ for every $x \in P$ and every $v \in \mathbb{S}^{d-1}$.

Proof. We may assume that $P$ is closed. Indeed, if the statement holds for $P$ closed, it extends immediately to countable unions of closed $P_{n}$ as follows: letting $S_{n}=\operatorname{NonD}\left(P_{n}\right)$ denote the collection of functions $f \in \operatorname{Lip}_{1}\left([0,1]^{d}\right)$ that are non-differentiable at every point of $P_{n}$ in the very strong sense described in the statement of the theorem, we get that each $S_{n}$ is residual. Hence,

$$
\left\{f \in \operatorname{Lip}_{1}\left([0,1]^{d}\right): \mathcal{D} f(x, v)=[-1,1] \text { for any } x \in \bigcup_{n=1}^{\infty} P_{n} \text { and } v \in \mathbb{S}^{d-1}\right\} \supseteq \bigcap_{n \geq 1} S_{n}
$$

is residual too.

Let $P \subseteq(0,1)^{d}$ be a closed, purely unrectifiable set and $S:=\operatorname{NonD}(P)$. We now consider a BanachMazur game $G_{B M}$, balls in $\operatorname{Lip}_{1}\left([0,1]^{d}\right)$ with the target set $S$ and show that Player II has a winning strategy; by Theorem 3.16, this will imply that $S$ is residual in $\operatorname{Lip}_{1}\left([0,1]^{d}\right)$.

Assume $H_{0}=(0,1)^{d}$. Fix a sequence $\left(e_{n}\right)$ of vectors with $\left\|e_{n}\right\|<1$ such that the collection $\left(e_{n}\right)$ is dense in the unit ball $B(0,1)$. Let $g_{0}(x)=0$ for all $x \in[0,1]^{d}$ and $\omega_{0}=1$.

On reaching step $n$ in the Banach-Mazur game, the two players would have constructed a nested sequence of open balls, and Player II would have additionally defined a nested sequence of open sets $H_{0} \supseteq \cdots \supseteq H_{n-1} \supseteq P$.

Assume $B\left(f_{n}, r_{n}\right)$ is the $n$th choice of Player I. Using that smooth functions are dense in $C\left([0,1]^{d}\right)$ followed by Lemma 3.17, we choose $f_{n}^{(1)} \in C^{1}\left([0,1]^{d}\right)$ such that $\operatorname{Lip}\left(f_{n}^{(1)}\right)<1$ and $\left\|f_{n}-f_{n}^{(1)}\right\|_{\infty}<$ $r_{n} / 2$. Choose $\eta_{n} \in\left(0,2^{-n}\right)$ s.t. $\max \left(\operatorname{Lip}\left(f_{n}^{(1)}\right),\left\|e_{n}\right\|\right)+\eta_{n}<1$. Let $\omega_{n}=\min \left(r_{n} / 2,2^{-n}\right)$. 
Apply now Lemma 5.2 to $P$ and $H:=H_{n-1}, \omega_{0}:=\omega_{n}, f:=f_{n}^{(1)}, e:=e_{n}$, and $\eta:=\eta_{n}$ to get function $g_{n}:=g:[0,1]^{d} \rightarrow \mathbb{R}, \xi_{n}:=\xi_{0}, \varepsilon_{n}:=r \in\left(0, \omega_{n}\right)$, and an open set $H_{n}:=U$.

From Lemma 5.2 (ii), we have that $g_{n} \in \operatorname{Lip}_{1}\left([0,1]^{d}\right)$ and $\left\|g_{n}-f_{n}^{(1)}\right\|_{\infty} \leq \omega_{n} \leq r_{n} / 2$, and hence $\left\|g_{n}-f_{n}\right\|_{\infty}<r_{n}$. Choose $\rho_{n} \in\left(0, \min \left(\xi_{n}, 2^{-n}\right)\right)$ such that $\bar{B}\left(g_{n}, \rho_{n}\right) \subseteq B\left(f_{n}, r_{n}\right)$. Let Player II's response be $B\left(g_{n}, \rho_{n}\right)$.

Since $\bar{B}\left(g_{n}, \rho_{n}\right) \subseteq B\left(g_{n-1}, \rho_{n-1}\right)$ and $\rho_{n} \rightarrow 0$, we conclude that the intersection of balls $B\left(g_{n}, \rho_{n}\right)$ is a single function $h \in \operatorname{Lip}_{1}\left([0,1]^{d}\right)$. We now show that $h$ has no directional derivatives at any $x \in P$ and, moreover, $\mathcal{D} h(x, v) \supseteq[-1,1]$ for every $x \in P$ and every $v \in \mathbb{S}^{d-1}$. As it is clear that $\mathcal{D} h(x, v) \subseteq[-1,1]$ from $\operatorname{Lip}(h) \leq 1$, this will imply the required equality.

Indeed, fix any $x \in P, v \in \mathbb{S}^{d-1}$, and $n \geq 1$. Recall the application of Lemma 5.2 that provided $g_{n}=g$ and $\xi_{n}=\xi_{0}$. Since $\|h-g\|_{\infty}=\left\|h-g_{n}\right\|_{\infty} \leq \rho_{n} \leq \xi_{n}=\xi_{0}$, we see that $h$ satisfies condition (iii) of Lemma 5.2. Hence, $\left|h(x+y)-h(x)-\left\langle e_{n}, y\right\rangle\right| \leq \eta_{n} \varepsilon_{n}$ whenever $\|y\| \leq \varepsilon_{n}$. In particular, letting $y=\varepsilon_{n} v$, we get

$$
\left|\frac{h\left(x+\varepsilon_{n} v\right)-h(x)}{\varepsilon_{n}}-\left\langle e_{n}, v\right\rangle\right| \leq \eta_{n} .
$$

As the vectors $e_{n}$ form a dense subset of the closed ball $\bar{B}(0,1), 0<\varepsilon_{n} \leq \omega_{n} \leq 2^{-n} \rightarrow 0$, and $0<\eta_{n} \leq 2^{-n} \rightarrow 0$, we get that $\mathcal{D} h(x, v) \supseteq[-1,1]$, and hence $\mathcal{D} h(x, v)=[-1,1]$.

\section{Comparison with vector-valued mappings}

For $d, l \in \mathbb{N}$, we denote by $\operatorname{Lip}_{1}\left([0,1]^{d}, \mathbb{R}^{l}\right)$ the space of Lipschitz mappings $f:[0,1]^{d} \rightarrow \mathbb{R}^{l}$ with $\operatorname{Lip}(f) \leq 1$, viewed as a complete metric space with the supremum metric. In most of the paper, we have $l=1$ and abbreviate $\operatorname{Lip}_{1}\left([0,1]^{d}, \mathbb{R}\right)$ to $\operatorname{Lip}_{1}\left([0,1]^{d}\right)$. Merlo [17] shows that whenever $d \leq l$ and $\mathscr{A} \subseteq(0,1)^{d}$ is a non-coverable set in the sense of Theorem 2.5, there is a residual set $S \subseteq \operatorname{Lip}_{1}\left([0,1]^{d}, \mathbb{R}^{l}\right)$ for which every mapping $f=\left(f_{1}, \ldots, f_{l}\right) \in S$ has a directional derivative in $\mathscr{A}$; see [17] Proposition 3.3 and Theorem 2.8. At first glance, it may appear that this statement is closely related to Theorem 2.5. Indeed, for such non-coverable $\mathscr{A} \subseteq(0,1)^{d}$ and residual $S \subseteq \operatorname{Lip}_{1}\left([0,1]^{d}, \mathbb{R}^{l}\right)$, the natural projection mappings

$$
\rho_{j}: \operatorname{Lip}_{1}\left([0,1]^{d}, \mathbb{R}^{l}\right) \rightarrow \operatorname{Lip}_{1}\left([0,1]^{d}, \mathbb{R}\right), \quad f=\left(f_{1}, \ldots, f_{l}\right) \mapsto f_{j},
$$

for $j=1, \ldots, l$, give rise to sets $\rho_{1}(S), \ldots, \rho_{l}(S) \subseteq \operatorname{Lip}_{1}\left([0,1]^{d}, \mathbb{R}\right)$ in which all functions have a directional derivative in $\mathscr{A}$. Since $S$ is residual in $\operatorname{Lip}_{1}\left([0,1]^{d}, \mathbb{R}^{l}\right)$, we might hope that the projections $\rho_{j}(S)$ are also large in some sense in $\operatorname{Lip}_{1}\left([0,1]^{d}, \mathbb{R}\right)$ and therefore hope to obtain via [17] a statement of the form of Theorem 2.5 with full differentiability weakened to the existence of a directional derivative. However, the next theorem demonstrates that this argument fails badly: even very large residual sets in $\operatorname{Lip}_{1}\left([0,1]^{d}, \mathbb{R}^{l}\right)$ may project to negligible sets in $\operatorname{Lip}_{1}\left([0,1]^{d}, \mathbb{R}\right)$. Thus, Theorem 2.5 and its implications in Theorems 2.1 and 2.2 are completely independent of [17] for all dimensions $d \geq 2$.

Theorem 6.1. Let $d, l \in \mathbb{N}$ with $l \geq 2$ and $\rho: \operatorname{Lip}_{1}\left([0,1]^{d}, \mathbb{R}^{l}\right) \rightarrow \operatorname{Lip}_{1}\left([0,1]^{d}, \mathbb{R}\right)$ be the standard projection defined by

$$
\rho(f)=f_{1}, \quad f=\left(f_{1}, \ldots, f_{l}\right) \in \operatorname{Lip}_{1}\left([0,1]^{d}, \mathbb{R}^{l}\right) .
$$

Then there exists an open, dense subset $U$ of $\operatorname{Lip}_{1}\left([0,1]^{d}, \mathbb{R}^{l}\right)$ for which the set $\rho(U)$ is of the first Baire category in $\operatorname{Lip}_{1}\left([0,1]^{d}, \mathbb{R}\right)$.

Note that Theorem 6.1 also provides an example of a residual subset $S$ of $\operatorname{Lip}_{1}\left([0,1]^{d}, \mathbb{R}\right)$ whose preimage $\rho^{-1}(S)$ under the projection $\rho$ is nowhere dense in $\operatorname{Lip}_{1}\left([0,1]^{d}, \mathbb{R}^{l}\right)$; we may take $S=$ $\operatorname{Lip}_{1}\left([0,1]^{d}, \mathbb{R}\right) \backslash \rho(U)$. For the proof of Theorem 6.1, we require two simple lemmata: 
Notation. In what follows, we use again the notation $I_{\eta}(t)$, introduced in Section 3, to denote the open interval $(t-\eta, t+\eta)$.

Lemma 6.2. Let $d, l \in \mathbb{N}, \gamma:[0,1] \rightarrow(0,1)^{d}$ be the length parameterisation of a line segment, $\mathcal{P}$ be a dense subset of $\operatorname{Lip}_{1}([0,1], \mathbb{R}), t_{0} \in(0,1), f=\left(f_{1}, \ldots, f_{l}\right) \in \operatorname{Lip}_{1}\left([0,1]^{d}, \mathbb{R}^{l}\right)$ be a mapping with $\operatorname{Lip}(f)<1, \varepsilon \in(0,1)$, and $j \in\{1, \ldots, l\}$. Then there exist $p \in \mathcal{P}, \eta>0$, and a mapping $g=\left(g_{1}, \ldots, g_{l}\right) \in \operatorname{Lip}_{1}\left([0,1]^{d}, \mathbb{R}^{l}\right)$ such that

(i) $\|g(x)-f(x)\| \leq \varepsilon$ for all $x \in[0,1]$.

(ii) $\left.g_{j} \circ \gamma\right|_{I_{\eta}\left(t_{0}\right)}=\left.p\right|_{I_{\eta}\left(t_{0}\right)}$.

(iii) $g_{1} \circ \gamma=p$ if $l=1$.

Proof. Let $\eta, \sigma>0$ be defined by

$$
\eta:=\frac{\left(1-\operatorname{Lip}(f)^{2}\right) \varepsilon^{2}}{128 \sqrt{d}}, \quad \sigma:=\left(\frac{8 \sqrt{d} \cdot \eta}{1-\operatorname{Lip}(f)^{2}}\right)^{1 / 2}=\frac{\varepsilon}{4},
$$

choose $p \in \mathcal{P}$ such that

$$
\left|p(t)-f_{j}(\gamma(t))\right| \leq \eta \quad \text { for all } t \in[0,1]
$$

and set

$$
J_{l}:= \begin{cases}I_{\eta}\left(t_{0}\right) & \text { if } l>1 \\ {[0,1]} & \text { if } l=1\end{cases}
$$

We define $g=\left(g_{1}, \ldots, g_{l}\right)$ initially on a subset of $[0,1]^{d}$ coordinatewise by

$$
\begin{aligned}
& g_{j}(x)= \begin{cases}p(t) & \text { if } x=\gamma(t), t \in J_{l}, \\
f_{j}(x) & \text { if } x \in[0,1]^{d} \backslash B\left(\gamma\left(J_{l}\right), \sigma\right), \text { and }\end{cases} \\
& g_{i}(x)= \begin{cases}f_{i}\left(\gamma\left(t_{0}\right)\right) & \text { if } x=\gamma(t), t \in J_{l}, \\
f_{i}(x) & \text { if } x \in[0,1]^{d} \backslash B\left(\gamma\left(J_{l}\right), \sigma\right) .\end{cases}
\end{aligned}
$$

for $i \in\{1, \ldots, l\} \backslash\{j\}$. The remainder of the proof is designed primarily for the more complicated case $l>1$. However, it also applies to the case $l=1$; observe that in this case, we necessarily have $j=1$, and all sums over $i \neq j$ disappear.

Note that $\left.g\right|_{[0,1]} \backslash B\left(\gamma\left(J_{l}\right), \sigma\right)$ and $\left.g\right|_{\gamma\left(J_{l}\right)}$ are 1-Lipschitz, where the latter case relies heavily on the fact that $\gamma$ is a length parameterisation of a line segment. To verify that this initially defined mapping is globally 1-Lipschitz on its entire domain, we observe, for $x=\gamma(t), t \in J_{l}$, and $y \in[0,1]^{d} \backslash B\left(\gamma\left(J_{l}\right), \sigma\right)$,

$$
\begin{aligned}
\|g(y)-g(x)\|^{2} & \leq \sum_{i \neq j}\left(\left|f_{i}(y)-f_{i}(x)\right|+\eta\right)^{2}+\left(\left|f_{j}(y)-f_{j}(x)\right|+\eta\right)^{2} \\
& \leq\|f(y)-f(x)\|^{2}+4 \sqrt{d} \cdot \eta+2 \eta^{2} \leq\left(\operatorname{Lip}(f)^{2}+\frac{8 \sqrt{d} \cdot \eta}{\sigma^{2}}\right)\|y-x\|^{2}=\|y-x\|^{2},
\end{aligned}
$$

using (6.2), $t \in J_{l}$, and (6.1). By Kirszbraun's theorem [12, Hauptsatz I], [8, 2.10.43], we may now extend $g$ to the whole of $[0,1]^{d}$ without increasing its Lipschitz constant. Thus, we obtain a mapping $g \in \operatorname{Lip}_{1}\left([0,1]^{d}, \mathbb{R}^{l}\right)$. Note that this mapping $g$ satisfies conclusions (ii) and (iii) of the lemma due to (6.3). To verify conclusion (i), we first note that the inequality of (i) is trivially valid for all $x \in$ $[0,1]^{d} \backslash B\left(\gamma\left(J_{l}\right), \sigma\right)$, where we have $f(x)=g(x)$. In the remaining case, $x \in B\left(\gamma\left(J_{l}\right)\right.$, $\left.\sigma\right)$, we may 
choose $t \in J_{l}$ with $\|x-\gamma(t)\| \leq \sigma$. We then derive

$$
\begin{aligned}
\|g(x)-f(x)\| \leq & 2 \sigma+\|g(\gamma(t))-f(\gamma(t))\| \\
& =2 \sigma+\left(\sum_{i \neq j}\left|f_{i}\left(\gamma\left(t_{0}\right)\right)-f_{i}(\gamma(t))\right|^{2}+\left|p(t)-f_{j}(\gamma(t))\right|^{2}\right)^{1 / 2} \leq 2 \sigma+\sqrt{d} \cdot \eta \leq \varepsilon,
\end{aligned}
$$

using (6.2), $t \in J_{l}$, and (6.1). This verifies (i) and completes the proof of the lemma.

Lemma 6.3. Let $f \in \operatorname{Lip}_{1}([0,1], \mathbb{R}), s<t \in[0,1], \tau, \varepsilon \in(0,1)$, and suppose that

$$
\|f(t)-f(s)\|=t-s .
$$

Then there exists $\delta>0$ such that for every $g \in \operatorname{Lip}_{1}([0,1], \mathbb{R})$ with $\|g-f\|_{\infty} \leq \delta$, the set

$$
C:=C_{g, \tau, s, t}=\left\{r \in[s, t]: g^{\prime}(r) \geq \tau\right\}
$$

has positive Lebesgue measure $\mathcal{L}(C) \geq(1-\varepsilon)(t-s)$.

Proof. We verify that the assertion of the lemma holds with

$$
\delta:=\frac{(1-\tau)(t-s) \varepsilon}{2} .
$$

Let $g \in \operatorname{Lip}_{1}([0,1], \mathbb{R})$ with $\|g-f\|_{\infty} \leq \delta$. Then

$$
\begin{aligned}
& t-s-2 \delta \leq g(t)-g(s)=\int_{s}^{t} g^{\prime}(r) d r \\
& \leq \int_{[s, t] \backslash C} g^{\prime}(r) d r+\int_{C} g^{\prime}(r) d r \leq \tau(t-s-\mathcal{L}(C))+\mathcal{L}(C) .
\end{aligned}
$$

Rearranging, we obtain

$$
\mathcal{L}(C) \geq t-s-\frac{2 \delta}{1-\tau}=(1-\varepsilon)(t-s) .
$$

We are now ready to prove Theorem 6.1 .

Proof of Theorem 6.1. Let $\mathcal{P}$ denote the set of piecewise isometric functions $[0,1] \rightarrow \mathbb{R}$ with only finitely many points of non-differentiability. Recall that $\mathcal{P}$ is a dense subset of $\operatorname{Lip}_{1}([0,1])$; see [22]. Let $\Omega$ denote the set of all mappings $f=\left(f_{1}, \ldots, f_{l}\right) \in \operatorname{Lip}_{1}\left([0,1]^{d}\right)$ for which there exist $t_{0} \in(0,1)$, $\eta>0$, and $p \in \mathcal{P}$ such that $\left.f_{2} \circ \gamma\right|_{I_{\eta}\left(t_{0}\right)}=\left.p\right|_{I_{\eta}\left(t_{0}\right)}$. By Lemma 6.2 , the set $\Omega$ is dense in $\operatorname{Lip}_{1}\left([0,1]^{d}, \mathbb{R}^{l}\right)$. We additionally fix a countable, dense subset $\Gamma$ of $\Omega$ and emphasise that $\Gamma$ is trivially also dense in $\operatorname{Lip}_{1}\left([0,1]^{d}, \mathbb{R}^{l}\right)$.

Let $f \in \Gamma$, and let $t_{0} \in(0,1), \eta>0$, and $p \in \mathcal{P}$ witness that $f \in \Omega$. Since $\left.f_{2} \circ \gamma\right|_{I_{\eta}\left(t_{0}\right)}=\left.p\right|_{I_{\eta}\left(t_{0}\right)}$ and $p \in \mathcal{P}$, there exist points $s_{f}<t_{f} \in I_{\eta}\left(t_{0}\right)$ such that $\left|f_{2} \circ \gamma\left(t_{f}\right)-f_{2} \circ \gamma\left(s_{f}\right)\right|=t_{f}-s_{f}$. Let $\delta_{f}>0$ be given by the conclusion of Lemma 6.3 applied to $f_{2} \circ \gamma \in \operatorname{Lip}_{1}([0,1], \mathbb{R}) s_{f}<t_{f}, \tau=3 / 4$, and $\varepsilon=1 / 4$. The required open dense subset of $\operatorname{Lip}_{1}\left([0,1]^{d}, \mathbb{R}^{l}\right)$ is now defined by

$$
U=\bigcup_{f \in \Gamma} B\left(f, \delta_{f}\right) .
$$


To verify that $\rho(U)$ is of the first Baire category in $\operatorname{Lip}_{1}\left([0,1]^{d}, \mathbb{R}\right)$, it suffices to show that each set $\overline{\rho\left(B\left(f, \delta_{f}\right)\right)}$ with $f \in \Gamma$ has an empty interior. We fix $f \in \Gamma$. First, observe that

$$
\overline{\rho\left(B\left(f, \delta_{f}\right)\right)}=\rho\left(\bar{B}\left(f, \delta_{f}\right)\right) .
$$

This follows immediately from the continuity of $\rho$ and the fact that $\bar{B}\left(f, \delta_{f}\right)$ is compact in $\operatorname{Lip}_{1}\left([0,1]^{d}, \mathbb{R}^{l}\right)$, where the latter is a consequence of the Arzelà-Ascoli theorem.

Assume that the set given in (6.4) has a non-empty interior. We complete the proof by deriving a contradiction. Fix a function $\widetilde{f} \in \operatorname{Int} \rho\left(\bar{B}\left(f, \delta_{f}\right)\right)$ with $\operatorname{Lip}(\widetilde{f})<1$. By Lemma 6.2 applied to $\widetilde{f} \in \operatorname{Lip}_{1}\left([0,1]^{d}, \mathbb{R}\right)$ and $l=1$, there exist $q \in \mathcal{P}$ and a function $g_{1} \in \rho\left(\bar{B}\left(f, \delta_{f}\right)\right)$ such that $g_{1} \circ \gamma=q$. Let $\left(g_{2}, \ldots, g_{l}\right) \in \operatorname{Lip}_{1}\left([0,1]^{d}, \mathbb{R}^{l-1}\right)$ be such that $\left(g_{1}, g_{2}, \ldots, g_{l}\right) \in \bar{B}\left(f, \delta_{f}\right)$. Then $\left\|g_{2} \circ \gamma-f_{2} \circ \gamma\right\|_{\infty} \leq$ $\delta_{f}$. Therefore, by the choice of $\delta_{f}$ and Lemma 6.3, we obtain a set

$$
C=C_{g_{2}, 3 / 4, s_{f}, t_{f}} \subseteq\left[s_{f}, t_{f}\right],
$$

of Lebesgue measure at least $(1-\varepsilon)\left(t_{f}-s_{f}\right)=3\left(t_{f}-s_{f}\right) / 4>0$, on which $g_{2} \circ \gamma$ is differentiable with $\left|\left(g_{2} \circ \gamma\right)^{\prime}(t)\right| \geq 3 / 4$ for all $t \in C$. However, at all but finitely many points $t \in[0,1]$, we have $\left|\left(g_{1} \circ \gamma\right)^{\prime}(t)\right|=\left|q^{\prime}(t)\right|=1$. Therefore, all but finitely many $t \in C$ satisfy

$$
\left|\left(g_{1} \circ \gamma\right)^{\prime}(t)\right|^{2}+\left|\left(g_{2} \circ \gamma\right)^{\prime}(t)\right|^{2} \geq 1+(3 / 4)^{2}>1 .
$$

Recalling that $\gamma$ is the length parameterisation of a line segment, we see that this is clearly incompatible with $g$ being 1-Lipschitz.

Acknowledgements. Michael Dymond acknowledges the support of the Austrian Science Fund (FWF): P $30902-N 35$ and of the EPSRC grant EP/N027531/1.

Olga Maleva acknowledges the support of the EPSRC grant EP/N027531/1.

The authors would like to thank David Preiss for very helpful and valuable discussions. We would also like to thank the organisers of the $48^{\text {th }}$ Winter School in Abstract Analysis, Czech Republic, and in particular Martin Rmoutil, for stimulating conversations.

Conflict of Interest: None.

\section{References}

[1] G. Alberti, M. Csörnyei, and D. Preiss, 'Differentiability of Lipschitz functions, structure of null sets, and other problems', in Proceedings of the International Congress of Mathematicians 2010 (ICM 2010) (In 4 Volumes) Vol. I: Plenary Lectures and Ceremonies Vols. II-IV: Invited Lectures (World Scientific, 2010), 1379-1394.

[2] S. Banach, 'Über die Baire'sche Kategorie gewisser Funktionenmengen', Studia Mathematica 3(1): 174-179, 1931.

[3] G. Choquet, 'Application des propriétés descriptives de la fonction contingent à la théorie des fonctions de variable réelle et à la géométrie différentielle des variétés cartésiennes', J. Math. Pures Appl. 9(26): 115-226 (1948), 1947.

[4] M. Csörnyei, D. Preiss, and J. Tiser, 'Lipschitz functions with unexpectedly large sets of nondifferentiability points', 2005, Abstract and Applied Analysis, (4), 361-373, 2005.

[5] M. Doré and O. Maleva, 'A universal differentiability set in Banach spaces with separable dual', Journal of Functional Analysis 261(6): 1674-1710, 2011.

[6] M. Dymond, 'Typical differentiability within an exceptionally small set', J. Math. Anal. Appl. 490 (2020), no. 2.

[7] M. Dymond and O. Maleva, 'Differentiability inside sets with Minkowski dimension one', Michigan Math. J. 65(3): 613636, 082016.

[8] H. Federer, Geometric Measure Theory, Classics in Mathematics, (Springer, 1996).

[9] S. Fitzpatrick, 'Differentiation of real-valued functions and continuity of metric projections', Proceedings of the American Mathematical Society 91(4): 544-548, 1984.

[10] E. Hewitt and K. Stromberg, Real and Abstract Analysis, (Springer-Verlag, New York-Heidelberg, 1975).

[11] A. Kechris, Classical Descriptive Set Theory, vol. 156 (Springer Science \& Business Media, 2012).

[12] M. Kirszbraun, 'Über die zusammenziehende und Lipschitzsche Transformationen', Fundamenta Mathematicae 22(1): 77 $108,1934$.

[13] C. Kuratowski, Topology I, (Academic Press New York and London, 1966). 
[14] E. Le Donne, A. Pinamonti, and G. Speight, 'Universal differentiability sets and maximal directional derivatives in Carnot groups', J. Math. Pures Appl. 9(121): 83-112, 2019.

[15] O. Maleva and D. Preiss, 'Cone unrectifiable sets and non-differentiability of Lipschitz functions', Israel Journal of Mathematics 232 (2019), no. 1, 75-108.

[16] P. Mattila, Geometry of Sets and Measures in Euclidean Spaces: Fractals and Rectifiability, Cambridge Studies in Advanced Mathematics (Cambridge University Press, 1995).

[17] A. Merlo, 'Full non-differentiability of typical Lipschitz functions', arXiv:1906.08366, 2019.

[18] G. Petruska, 'On Borel sets with small cover: A problem of M. Laczkovich', Real Analysis Exchange 18(2): 330-338, 1992.

[19] A. Pinamonti and G. Speight, 'A measure zero universal differentiability set in the Heisenberg group', Math. Ann. 368(1-2): 233-278, 2017.

[20] D Preiss. 'Differentiability of Lipschitz functions on Banach spaces', Journal of Functional Analysis 91(2): 312-345, 1990.

[21] D. Preiss and G. Speight, Differentiability of Lipschitz functions in Lebesgue null sets', Invent. Math. 199(2): 517-559, 2015.

[22] D. Preiss and J. Tišer, 'Points of non-differentiability of typical Lipschitz functions', Real Analysis Exchange 20(1): 219-226, 1994.

[23] S. Rădulescu and M. Rădulescu, 'Local inversion theorems without assuming continuous differentiability', J. Math. Anal. Appl. 138(2): 581-590, 1989.

[24] S. Solecki, 'Covering analytic sets by families of closed sets', J. Symbolic Logic 59(3): 1022-1031, (09) 1994.

[25] Z. Zahorski, ' Sur les ensembles des points de divergence de certaines intégrales singulières', Ann. Soc. Polon. Math. 19: 66-105 (1947), 1946. 